\title{
Position Estimation of Mobile Robots Using Omni-Directional Cameras
}

by

\section{Omar Mohamed Mostafa Kamal Fouad}

\author{
A Thesis submitted to \\ the Faculty of Graduate Studies and Research \\ in partial fulfilment of \\ the requirements for the degree of \\ Master of Applied Science \\ in \\ Mechanical Engineering \\ Carleton University \\ Ottawa, Ontario, Canada \\ September 2014
}

Copyright (C)

2014 - Omar Mohamed Mostafa Kamal Fouad 


\section{Abstract}

Mobile robots are prospected to significantly improve the efficiency and productivity of individuals. However, to fulfil their required tasks, they must be able to efficiently manoeuvre in their surrounding environments. This requirement necessitates that these robots posses the ability to acquire their current position and orientation within their work space. To acquire this information, mobile robots need precise and reliable perception techniques. Different perception methods has been described in literature and can be categorized based on their way of interaction with their given environments, i.e., passive or active sensors. These methods map the robot environment and identify its position and orientation within the robot workspace.

In this work, a design of a perception approach for indoor service mobile robots is considered, i.e, robotic vacuum cleaners. Unlike outdoor environments, in which Global Positioning System (GPS) can be utilized, indoor environments usually include small workspaces with complex details. Thus, a significantly higher localization precision is required. Readily available sensing techniques that meet those requirements utilize sensors such as transceivers and vision systems. These perception approaches depend on the workspace type. In the proposed approach, a stereo vision system has been used. Such an approach captures the environment features to produce a 2D/3D map. However, due to the mobility of indoor robots, the problem of losing environment features arises, e.g., they might encounter scenes with non-detectable features. Since the proposed application for this work is an intelligent indoor robotic vacuum 
cleaner operating in a highly packed environment, it is necessary for the robot to continuously adopt a highly precise floor map.

In order to solve the difficulty of obtaining a high-precision map in a highly compact environment, a stereo panoramic vision perception model was developed to integrate the data from two omni-directional vision sensors. By applying this data in the proposed geometric model, a mobile robot can obtain a highly precise map of its surrounding environment. In particular, the proposed depth estimation technique allows robotic vehicles to instantly and reliably recover their position and orientation within the surrounding environment. In addition, a new constrained calibration and rectification method for the proposed setup of two side-to-side panoramic lenses has been proposed. 
I dedicate this work to my parents, my brother, and my beloved wife Sarah to whom I owe everything I have reached. I cannot be thankful enough for their pure love, guidance, and support in every single moment of my life. 


\section{Acknowledgments}

Before beginning, I am extremely grateful to Allah for allowing me to reach this point in pursuing my goals. I am also extremely thankful for my supervisors Professor Jie Liu and Professor Jurek Sasiadek for their continuous support and encouragement along the Masters' journey. I am also thankful for my colleagues in the research lab for their encouragement and insightful suggestions for improving this work.

I would like to express my gratitude for the defense committee for giving me their

precious time to allow me to present my work and for their valuable comments and discussions on improving this thesis.

Finally, I would like to acknowledge the financial support from my supervisor and Carleton University to myself and the research project that allowed the further improvement of this work. 


\section{Table of Contents}

Abstract $\quad$ ii

Acknowledgments $\quad$ V

Table of Contents $\quad$ vi

List of Figures $\quad$ ix

1 Introduction 1

1.1 Mobile Robots . . . . . . . . . . . . . . . 1

$1.2 \quad \mathrm{SLAM} \ldots \ldots \ldots \ldots \ldots \ldots$

1.2 .1 Mapping . . . . . . . . . . . . . . . . . . . 3

1.2.2 Localization . . . . . . . . . . . . . . . 4

1.3 Path Planning . . . . . . . . . . . . . . . . 4

1.4 Thesis Objective . . . . . . . . . . . . . . . 5

1.5 Thesis Contributions ................... 6

1.6 Thesis Organization . . . . . . . . . . . . . . . . . 7

2 Perception and Stereo Vision Fundamentals $\quad 8$

2.1 Introduction . . . . . . . . . . . . . . . . 8

2.2 Active Sensors . . . . . . . . . . . . . . . . . 8

2.2.1 Transceivers ................... 8 
2.3 Passive sensors . . . . . . . . . . . . . . . . . . . . . . . . 10

2.3.1 Tactile Sensor . . . . . . . . . . . . . . . . 10

2.3.2 Computer Vision . . . . . . . . . . . . . . . . . . . . . . 11

2.3.3 Stereo Vision . . . . . . . . . . . . . . . . . . 12

2.4 Visual Odometry . . . . . . . . . . . . . . . . . . . 18

3 Literature Review $\quad 19$

3.1 Introduction . . . . . . . . . . . . . . . . . . . . . 19

3.2 Panoramic and Omni-directional Lenses . . . . . . . . . . . . 20

3.2.1 Fish-Eye Lenses . . . . . . . . . . . . . . . . . . . . . . 20

3.2 .2 Catadioptric Lenses . . . . . . . . . . . . . . . . . 21

3.3 Folded Panoramic Lenses . . . . . . . . . . . . . . . . . . . . . 24

3.4 Omni-directional Camera Calibration . . . . . . . . . . . . 24

3.4.1 Single Camera Calibration . . . . . . . . . . . . . . 24

3.4.2 Stereo Calibration . . . . . . . . . . . . . . . 25

3.5 Mobile Robots Positioning . . . . . . . . . . . . . . . . . . 27

3.6 The Proposed Approach . . . . . . . . . . . . . . . . . . . . 32

3.6.1 Calibration . . . . . . . . . . . . . . . . . 32

3.6.2 Stereo Correspondence and Positioning . . . . . . . . . . . 33

4 Panoramic Camera Calibration 35

4.1 Panoramic Image Unwrapping . . . . . . . . . . . . . . . . . . . 35

4.1.1 Circular Panoramic Image Parameters . . . . . . . . . . . . 36

4.1 .2 Image Unwrapping . . . . . . . . . . . . . . . . . 38

4.1 .3 Experiments ....................... 41

4.2 Geometric Model . . . . . . . . . . . . . . . . . . . 43

4.3 Calibration ........................ 46

4.3.1 Experiments .................... 51 
5 Stereo Panoramic Imaging $\quad 58$

5.1 Introduction . . . . . . . . . . . . . . . . . . . 58

5.1 .1 Coaxial Format . . . . . . . . . . . . . . . . . 58

5.1 .2 Side-To-Side Format . . . . . . . . . . . . . . . . 59

5.2 Epipolar Geometry . . . . . . . . . . . . . . . . . . . 59

5.3 Stereo Transformation . . . . . . . . . . . . . . . . . . . 64

5.3.1 Rectification . . . . . . . . . . . . . . . . 69

5.4 Simulations and Experiments . . . . . . . . . . . . . . 69

5.4 .1 3D Print Material . . . . . . . . . . . . . . . . 69

5.4 Resolution Effects . . . . . . . . . . . . . . . 70

5.4.3 Angular Step Size and Depth Estimation Performance . . . . 73

5.4.4 Distance Measurement . . . . . . . . . . . . . . . . 75

6 Summary, Future Work and Conclusions $\quad 81$

6.1 Summary .............................. . . 81

6.1.1 Chapter $1 \ldots \ldots . \ldots . \ldots 1$

6.1 .2 Chapter $2 \ldots \ldots \ldots$. . . . . . . . . . . . . 81

6.1.3 Chapter $3 \ldots \ldots \ldots . \ldots \ldots 2$

6.1 .4 Chapter $4 \ldots \ldots \ldots$. . . . . . . . . . . . . . 82

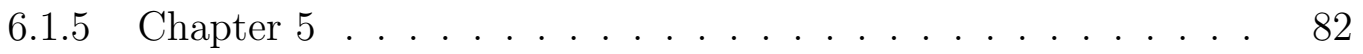

6.2 Future Work . . . . . . . . . . . . . . . . . . 83

6.3 Conclusions .......................... 84

$\begin{array}{lr}\text { Appendices } & 86\end{array}$

A Code for Unwrapping Panoramic Images and Defining the Epipolar $\begin{array}{lr}\text { Curves of Stereo Panoramic Images. } & 87\end{array}$

$\begin{array}{ll}\text { List of References } & 109\end{array}$ 


\section{List of Figures}

1 Mobile Robot . . . . . . . . . . . . . . . . . . 2

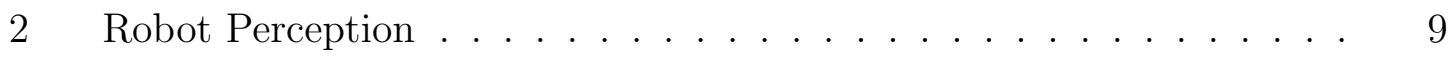

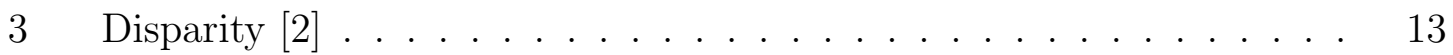

4 Stereo Cameras $[2] \ldots \ldots \ldots \ldots \ldots$

5 Epipolar Geometry $[2] \ldots \ldots \ldots \ldots \ldots$

6 Essential Geometry $[2] \ldots \ldots \ldots \ldots \ldots \ldots$

$7 \quad$ Calibration And Rectification $[2] \ldots \ldots \ldots \ldots$

8 Panoramic Images $[10] \ldots \ldots \ldots$

9 Jollystar Fisheye Lens _. . . . . . . . . . . . . . . . . . . . . 21

10 Single Mirror Panoramic Lens $[11] \ldots \ldots \ldots \ldots$

11 Panoramic Mirrors $[12] \ldots \ldots \ldots \ldots \ldots$

12 Folded Panoramic Lens $[9] \ldots \ldots \ldots \ldots$

13 Image Unwrapping $[13] \ldots \ldots \ldots \ldots$

14 Hough Line Transform . . . . . . . . . . . . . . . . . 37

15 Application of Hough Circle Transform . . . . . . . . . . . 38

16 Circular Geometry of Omni-directional Images . . . . . . . . . . . 39

17 Image Unwrapping Flowchart . . . . . . . . . . . . . . . . 41

18 Source Images $\ldots \ldots \ldots \ldots$

19 Unwrapped Images $\ldots \ldots \ldots$

20 Single Camera Geometric Model . . . . . . . . . . . . . . . . . 45 
21 Vertical Angular Steps . . . . . . . . . . . . . . . . . . 46

22 Calibration Board . . . . . . . . . . . . . . . . 47

23 Horizontal Calibration Board Lines . . . . . . . . . . . . . . 48

24 Horizontal Lines Perspectives . . . . . . . . . . . . . . . . . 49

25 Image Parameters detection simulation $\ldots \ldots \ldots \ldots \ldots \ldots$

26 Raspberry-Pi Camera Module . . . . . . . . . . . . . . . . 52

27 Kogeto Dot Panoramic Lens . . . . . . . . . . . . . . . . 53

28 First Camera Intrinsic Parameters . . . . . . . . . . . . . . 55

29 Second Camera Intrinsic Parameters _. . . . . . . . . . . . 56

30 Calibration Flowchart _. . . . . . . . . . . . . 57

31 Epipolar Geometry Model - Coaxial Format . . . . . . . . . . . . 59

32 Epipolar Geometry Model - Side-To-Side Format . . . . . . . . . . 60

33 Panoramic Epipolar Geometry Model . . . . . . . . . . . . . . 63

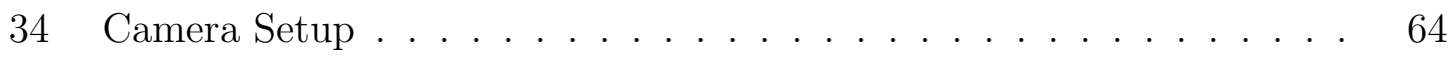

35 Corners Mask . . . . . . . . . . . . . . . . . . . . 64

36 Detailed Geometric Model - Translation _. . . . . . . . . . 66

37 Detailed Geometric Model - Rotation . . . . . . . . . . . . . . 67

38 Stereo Camera Setup Before Being Applying Dark Paint . . . . . . 70

39 Effect of Applying Dark Paint on Enhancing Captured Image Quality 71

40 High Quality Image . . . . . . . . . . . . . . . . . 72

41 Found Corners From iPhone Camera . . . . . . . . . . . . . . 72

42 Found Corners From Raspberry-Pi Camera . . . . . . . . . . . . . . 72

43 Angular Steps and Depth Estimation Errors . . . . . . . . . . 73

44 Maximum Depth Estimation Errors $($ at $d=100) \ldots \ldots . \ldots 74$

45 Maximum Depth Estimation Errors $($ at $d=200) \ldots \ldots \ldots$

46 Defined Distance Measurement _. . . . . . . . . . . 75

47 Robot at Distance 3.2 Meters . . . . . . . . . . . . . 76 
48 Epipolar Geometry Simulation - 3.2 Meters . . . . . . . . . . . . . . 76

49 Robot at Distance 2.1 Meters . . . . . . . . . . . . . . . . 77

50 Epipolar Geometry Simulation - 2.1 Meters . . . . . . . . . . . . . . . 77

51 Robot at Distance $90 \mathrm{~cm} \ldots \ldots \ldots \ldots$

52 Epipolar Geometry Simulation - $90 \mathrm{~cm} \ldots \ldots$. . . . . . . . . 78

53 Robot at Distance $30 \mathrm{~cm}$. . . . . . . . . . . . . . . . . . 79

54 Epipolar Geometry Simulation - $30 \mathrm{~cm}$. . . . . . . . . . . 79

55 Epipolar Geometry Simulation - $215 \mathrm{~mm}$. . . . . . . . . . . . 80 


\section{Chapter 1}

\section{Introduction}

\subsection{Mobile Robots}

Robots are machines that have different structures and mechanisms, e.g., stationary or mobile. A stationary robot is a machine that has a fixed physical location, whereas a mobile robot is an automatic machine that has a locomotive mechanism. There are different types of mobile robots depending on the manner through which they are controlled. In particular, mobile robots can be either autonomous or teleoperated. Autonomous robots can navigate inside their workspace without human intervention. On the other hand, tele-operated robots are remotely operated by humans. In the presented work hereinafter, an autonomous Unmanned Ground Vehicle (UGV) is considered. UGVs are categorized by their locomotion mechanisms, e.g., legged or wheeled. In this work, the considered UGV is assumed to have a wheeled mechanism that is composed of two independently driven wheels with one unpowered rear wheel as shown in Figure 1.

During the last five decades, mobile robots went through evolutionary changes that significantly broadened their spectrum of applications. Some of these applications involve robotic interactions with day-to-day human activities that subsequently increases humans productivity. Some examples include space exploration, mining, 


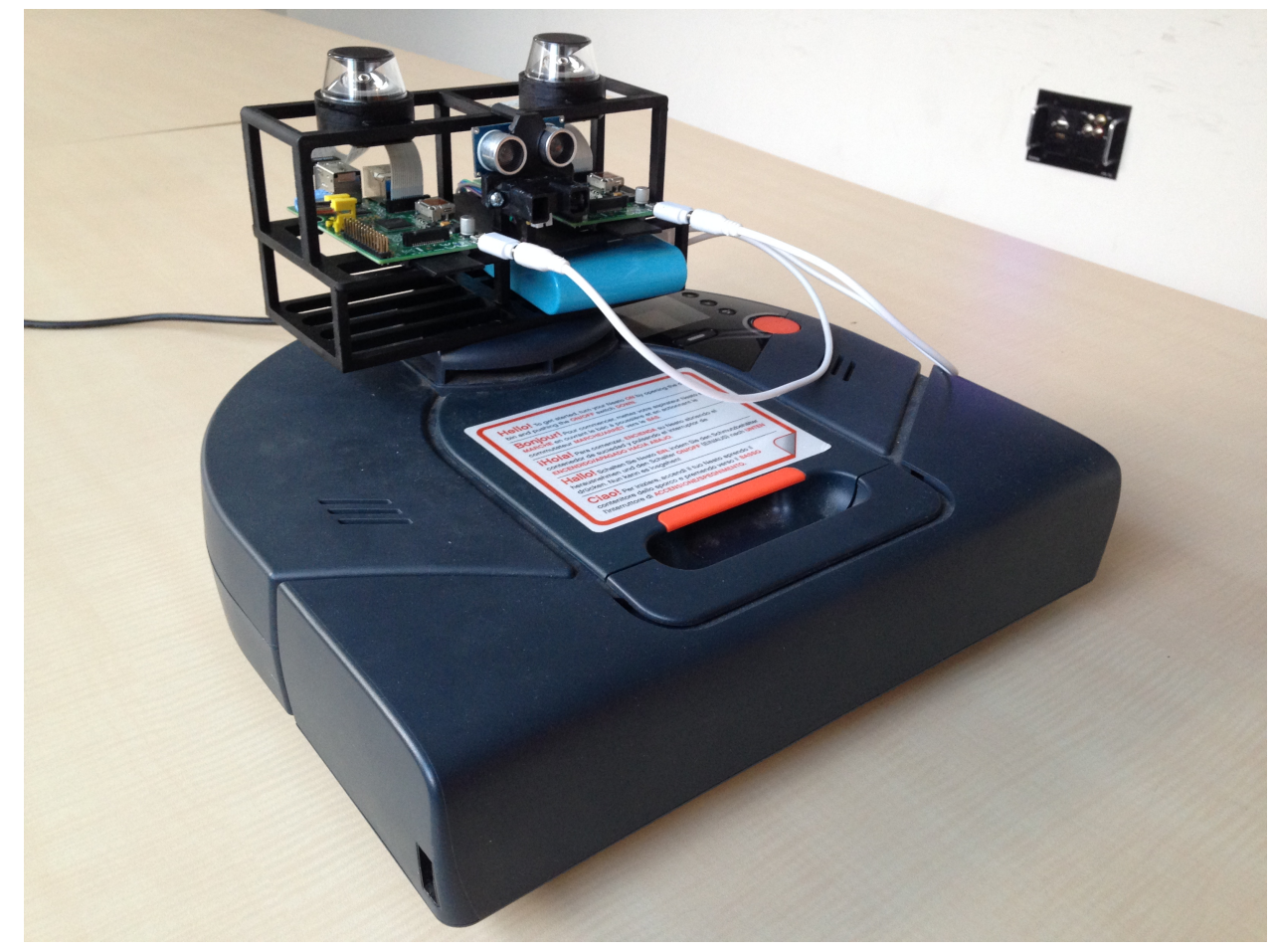

Figure 1: Mobile Robot

security, defense, and service robotic applications, e.g., robotic vacuum cleaning.

To efficiently fulfill its required task, a mobile robot must posses certain electrical and mechanical characteristics. This requires extensive multi-disciplinary research activities. In particular, structural and electrical platform design research is essential to provide mobile robots with the designated tools for achieving their tasks under multiple constraints such as weight, size and reliability. Furthermore, research in electrical control approaches, e.g., navigation systems, is necessary to enable a mobile robot to maneuver around obstacles and subsequently adapt to its surrounding environment. This research category can be divided into multiple subcategories, i.e., perception, Simultaneous Localization And Mapping (SLAM), and path planning. Since the focus of the proposed work is on mobile robot navigation, a brief introduction on some of these subcategories is provided next. 


\section{$1.2 \quad$ SLAM}

The objective of a mobile robot navigation system is to continuously localize the robot with respect to its provided workspace. In scenarios wherein the environment is static, a robot might have the workspace map readily available. However, in typical scenarios, a mobile robot usually operates in various dynamic environments. Therefore, a mobile robot not only needs to continuously construct and update its workspace map, but also needs to continuously localize itself within its established map. In particular, the accurate workspace mapping and robot localization provide the robot with the ability to successfully apply a path plan to fulfill its objective while avoiding obstacles.

\subsubsection{Mapping}

Robots strive to gather information about their workspace to enable their successful operation using what is called mapping. In particular, mapping is the process where robots, continuously, construct their workspace map during operation. In order to reliably map their workspace, robots require to, continuously, define the existence of the workspace features. This process uses the robot allothetic and idiothetic information sources to sense the environment information.

\section{Allothetic Sources}

Allothetic mapping data are the data read from the physical workspace environment. One example of allothetic sources would be the robot perception sensor, e.g., laser scanners. The perception sensors process and transform the environment information to useful signals, i.e., producing a usable 2D or 3D workspace map from the sensed data. Robot perception is essential for all autonomous mobile robot platform. 


\section{Idiothetic Sources}

Mobile robots idiothetic sources of environment information are represented in the robot's retrieved odometry data. Such information can provide the robot with its absolute position. However, odometry data are subject to high level of cumulative errors, e.g., slipping. These errors significantly increase as the robot operates.

\subsubsection{Localization}

Localization is the ability to know the exact location within a given map. When performing localization, robot can either use a preloaded or a generated map. However, in dynamic environments, robots usually use a generated map. Using this map, a mobile robot performs the localization based on the feedback of its allothetic and/or idiothetic mapping sources of information.

\section{Probabilistic Robot Localization}

Probabilistic robot localization is an approach used for reducing the localization

errors. Such method uses the different types of environment data along with state estimation filters and techniques to fulfill the mapping requirement while mitigating sensing errors during operation.

\subsection{Path Planning}

Path planning is a high level robot controlling technique that totally depends on the robots' functionality and objective. Robot path planning control can be divided in three stages as follows:

- Mission planner: Mission planner is the top level control for mobile robots. 
- Global planner: Global planner is considered as a high level intelligence. It is concerned with generating the path required by the robot in order to finish the required part of the mission.

- Local planner: Local planners are the lowest intelligence level of the mobile robot path planning task. They are concerned with obstacle avoidance and making sure the robot is following its global plan objectives.

\subsection{Thesis Objective}

In this thesis, the problem of indoor mobile robots positioning is considered. In particular, a novel approach for positioning robotic vacuum cleaners using a panoramic stereo imaging setup is proposed. The thesis objectives are summarized by the following points:

1. Design a low profile position estimation system to meet the design constraints of robotic vacuum cleaners. In particular, robotic vacuum cleaners are required to maneuver below short objects, and thus it has a strict constraint on platform height. In order to meet this constraint, a side-to-side stereo panoramic imaging setup is used for position estimation.

2. Derive a geometric model for panoramic cameras. This model is necessary for the calibration of panoramic cameras and the construction of the epipolar geometry of the provided stereo setup.

3. Modify the standard calibration board to improve the quality of the camera calibration process.

4. Provide a calibration algorithm for panoramic cameras to identify their intrinsic parameters. 
5. Derive the epipolar geometry model of the provided setup of stereo panoramic cameras.

6. Derive a depth estimation error equation for the proposed stereo vision setup to identify the sources of depth estimation errors.

7. Conduct simulations and physical experiments to verify the proposed calibration algorithm, epipolar geometry, and depth estimation analysis.

\subsection{Thesis Contributions}

In this thesis, an approach for the calibration and usage of omni-directional cameras in low profile indoor mobile robot applications is provided. The contributions of this thesis can be summarized as follows:

- Using the spherical geometry of panoramic cameras, a calibration algorithm is developed to identify the intrinsic camera parameters. This algorithm is novel in two aspects:

1. It uses a specially designed calibration board to increase the quality of the calibration process.

2. It imposes a constraint on the calibration board orientation to reduce the number of calibration parameters and subsequently reduce the calibration complexity.

- An epipolar geometry model is developed for the proposed stereo panoramic side-to-side imaging setup. This model was inspired by the spherical geometry and verified by real life experiments.

- Depth estimation analysis is performed for positioning the observed 3D scene features. In particular, an equation is derived to evaluate the depth estimation 
errors of the proposed side-to-side panoramic imaging setup. In addition, this equation enables the identification of the depth error sources. This equation is verified by both, simulations and real life experiments.

\subsection{Thesis Organization}

This thesis is organized as follows.

- Chapter two provides a review of the different types of environment perception approaches as well as a detailed introduction to stereo vision systems.

- Chapter three presents a detailed introduction on the panoramic imaging systems and their relevant literature review.

- Chapter four introduces a constrained calibration approach for panoramic cameras to identify their intrinsic parameters.

- Chapter five provides information about the different types of panoramic stereo vision systems and details on the rectification approach of the proposed stereo setup. In addition, this chapter, also, introduces details on the proposed epipolar geometry. Moreover, depth estimation analysis is conducted to show the feasibility of the proposed setup. Finally,relevant simulations and experiments on the proposed panoramic stereo imaging setup are conducted using real images. 


\section{Chapter 2}

\section{Perception and Stereo Vision}

\section{Fundamentals}

\section{$2.1 \quad$ Introduction}

Robot perception represents the robot's ability to sense its environment. There are different types of sensors used for robot perception, such as active and passive perception sensors. Active perception sensors provide their own energy to sense their environment. In particular, active sensors impose energy to the environment and use the received reflection to specify an environment property. Unlike active sensors, passive sensors captures naturally available energy from the surrounding environment. Using the data acquired by such sensors, a mobile robot can implement the essential environment perception and mapping needed for its successful operation.

\section{$2.2 \quad$ Active Sensors}

\subsubsection{Transceivers}

Transceivers are active sensors that are composed of a transmitter and a receiver on a single board. When used in mobile robots, these sensors identify the surrounding 


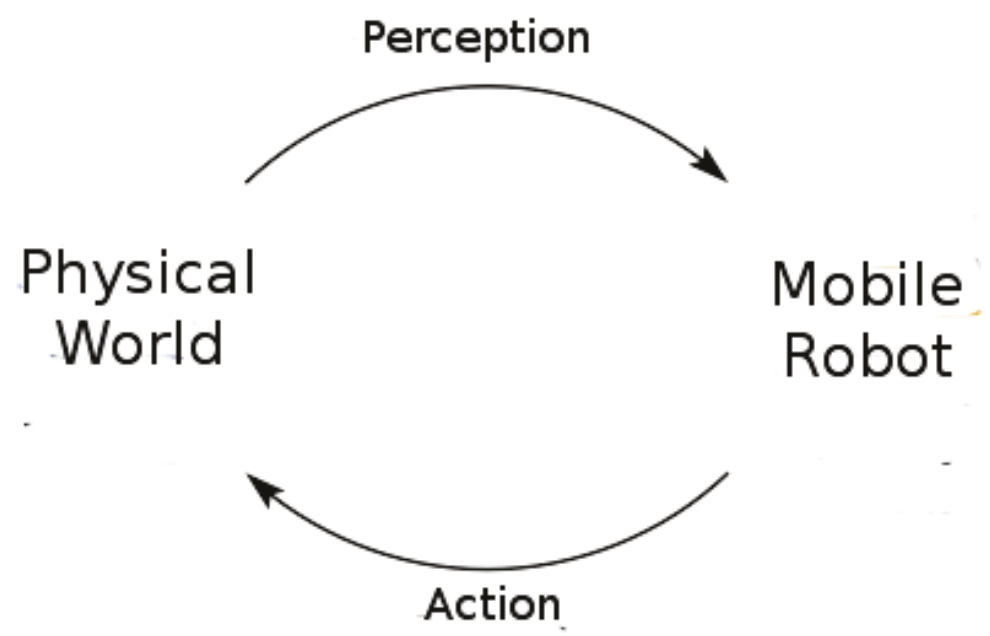

Figure 2: Robot Perception

environment by detecting the presence of objects and sometimes they, also, capture their distances from the sensor. To do this, transceivers measure specific parameters, e.g., the time of flight. In particular, transceivers measure the time interval between the transmission of a signal and the reception of its reflection. With a known signal speed, this time interval is directly proportional to the distance between an object and the sensor. Based on the nature of the transmitted signal, transceivers can be divided into the following subcategories:

Sonar Range Finders: In these sensors, the underlying transmitted signal is a mechanical wave with a frequency higher than $20 \mathrm{KHz}$ and a speed of $340 \mathrm{~m} / \mathrm{s}$. One example of the sonar range finders can be the ultrasound transceivers. However, due to their underlying mechanical wave structure, these sensors incur two main sources of distortion. First, sound absorbing materials significantly reduce the power of the transmitted wave because they work as dampers for sound waves. Consequently, the reflection of the transmitted signal might not be sensed by the receiver and thus the object might not be detected. Second, sonar signals have a wide field of view, i.e., the transmitted signal is dispersed over a wide angle. Thus, the transmitted 
signal might encounter multiple angles of reflection and/or deflection when confronted with irregular surface patterns. Consequently, each of these reflected signals incurs a different time of arrival. This phenomenon is referred to as ghost signals.

Infrared Range Finders: Unlike the sonar waves, infrared signals are invisible light waves with a frequency range of $300 \mathrm{GHz}$ to $430 \mathrm{THz}$ at a speed of 300,000 $\mathrm{km} / \mathrm{s}$. There are two types of infrared sensors, i.e., quantum infrared sensors and thermal infrared sensors. Quantum infrared sensors are wavelength dependent while the thermal infrared sensors are power dependent. Infrared range finders use quantum infrared transceivers to reduce environment noise effect by sending and detecting a specific signal wavelength. The quality of the infrared transceiver is dependent on the transceiver setup, the power of the used signal, and the field of view of the transmitted signal. Infrared transceivers are subject to different sources of infrared distortions. The received signal can be distorted with infrared radiations, light sources, or even heat sources. In addition, infrared light beams does not reflect with the same angle of surfaces with different colors and shades. However, some solutions have been developed for infrared transceivers to overcome such problems. The most advanced infrared transceivers use laser infrared beams that are optically focused and amplified at high frequency. These beams have a narrow and precise field of view that provides the transceiver with a highly accurate environment information. One example is the Light Detection And Ranging sensor(LiDAR) which provides precise and reliable environment scan in either 2D or 3D based on the sensor structure.

\subsection{Passive sensors}

\subsubsection{Tactile Sensor}

Tactile sensors are pressure sensors that gather information from the physical 
interaction with the environment. For example, tactile sensors are used in robotic vacuums in the form of a limit switch mounted in the robot bumper. Tactile sensors, in mobile robots, are usually used as a supportive sensation element to prevent destructive robot motion.

\subsubsection{Computer Vision}

Computer Vision is the process of acquiring 2D images of a $3 \mathrm{D}$ scene to generate useful environment information. In computer vision systems, cameras are the sensing modules that acquire the input data of a computer vision system. Computer vision techniques may be used for environment mapping in mobile robot applications. In particular, it would transform the 2D image perspectives of a 3D scene, captured from a setup of camera modules, to a 2D map or 3D point cloud. Consequently, the robot might use the generated map for navigation and obstacle avoidance purposes.

Computer vision is a computationally expensive solution for environment mapping. However, with nowadays technologies, computer vision is an affordable solution that can be used for robot perception. Computer vision systems have attracted increasing research attention because they have a high potential of mimicking human perception techniques.

Cameras: Cameras are composed of a 2-D array of light sensors. Unlike transceivers, cameras are considered passive sensors because they do not transmit signals, but instead capture the available light energy from the environment. Stereo Cameras are widely used for environment mapping by creating 3-D point clouds. The technology behind stereo cameras is based on defining and matching the correspondent observed feature from multiple perspectives. Stereo Cameras mapping algorithm triangulates the matched features in order to identify the depth of the observed feature with respect to the camera setup. For mapping purposes, two stereo 
camera setups are commonly used. One is the conventional stereo camera setup that is composed of two conventional cameras facing an object or a set of features from different perspectives. In the second setup, the stereo cameras are used with omnidirectional lenses. For reliable operation, stereo vision systems require calibration and rectification processes. The conventional stereo vision systems are described next.

\subsubsection{Stereo Vision}

Stereo cameras are mult-viewpoint vision systems that mimic the human approach of environment mapping and modeling. Their concept is based on using multiple images with different perspectives to observe a 3D scene. Further processing is done on the images to find the corresponding feature on both images. Despite of being computationally expensive, the search for the corresponding image feature can be simplified using some techniques such as calibration and rectification. After defining the corresponding feature in the stereo images, a technique, namely triangulation, is used to find the depth of a feature in the 3D environment.

\section{Triangulation}

Triangulation is the process of using a predefined feature correspondence and camera orientations to determine the depth of this feature with respect to the stereo setup in a 3D workspace. For ease of exposition, perfectly aligned and undistorted images are used to present the concept of triangulation as depicted in Figure 3. In this figure, $\left(x_{r}, y_{r}\right)$ correspond to a pixel coordinates in the right image frame, and $\left(x_{l}, y_{l}\right)$ correspond to a pixel coordinates in the left image frame. In the same figure, $d$ is the disparity between the two perspectives of a feature in the two image frames. The value of $d$ can be evaluated by the following equation

$$
d=x_{l}-x_{r}
$$




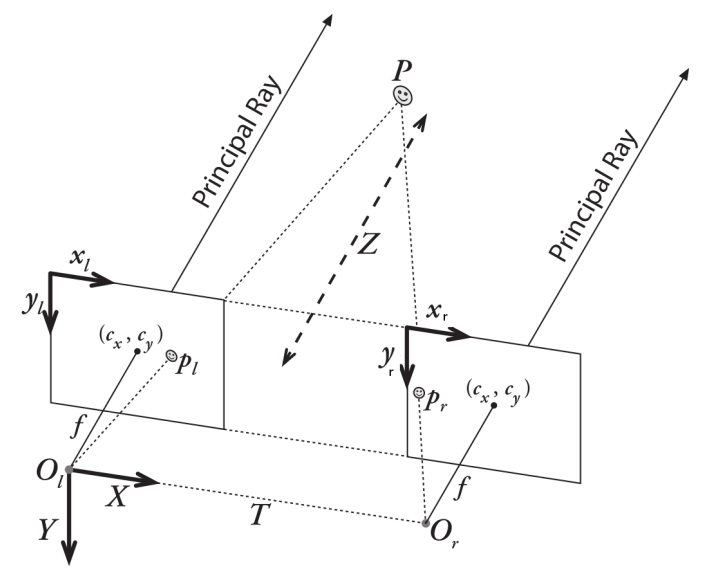

Figure 3: Disparity [2]

However, if the two cameras principal rays do intersect at a finite distance, the feature disparity can be evaluated by

$$
d=\left(x_{l}-c_{x l}\right)-\left(x_{r}-c_{x r}\right)
$$

where $c_{x l}$ and $c_{x r}$ are the points of intersection between the principle rays of the two perspectives and the left and right image frames, respectively.

The depth of a feature in the $3 \mathrm{D}$ environment, i.e., $Z$, is inversely proportional with the disparity of the feature correspondence. From the provided setup, there is a proportionality between the triangles of vertices $P x_{l} x_{r}$ and vertices $P_{x} O_{l} O_{r}$. Therefore, a relation between the $2 \mathrm{D}$ feature disparity and the $3 \mathrm{D}$ feature depth is provided where $f$ is the cameras' focal length, $O_{l}$ and $O_{r}$ are the centers of projections, and $T$ is the physical distance between the two cameras.

$$
\frac{T-d}{Z-f}=\frac{T}{Z} \Longrightarrow Z=\frac{f T}{d}
$$

In the non-ideal case, the previous equations will not be valid without calibration and rectification. Practically, stereo images are subject to distortions and 3D rotation. Therefore, stereo images need to be calibrated and rectified before further processing. 


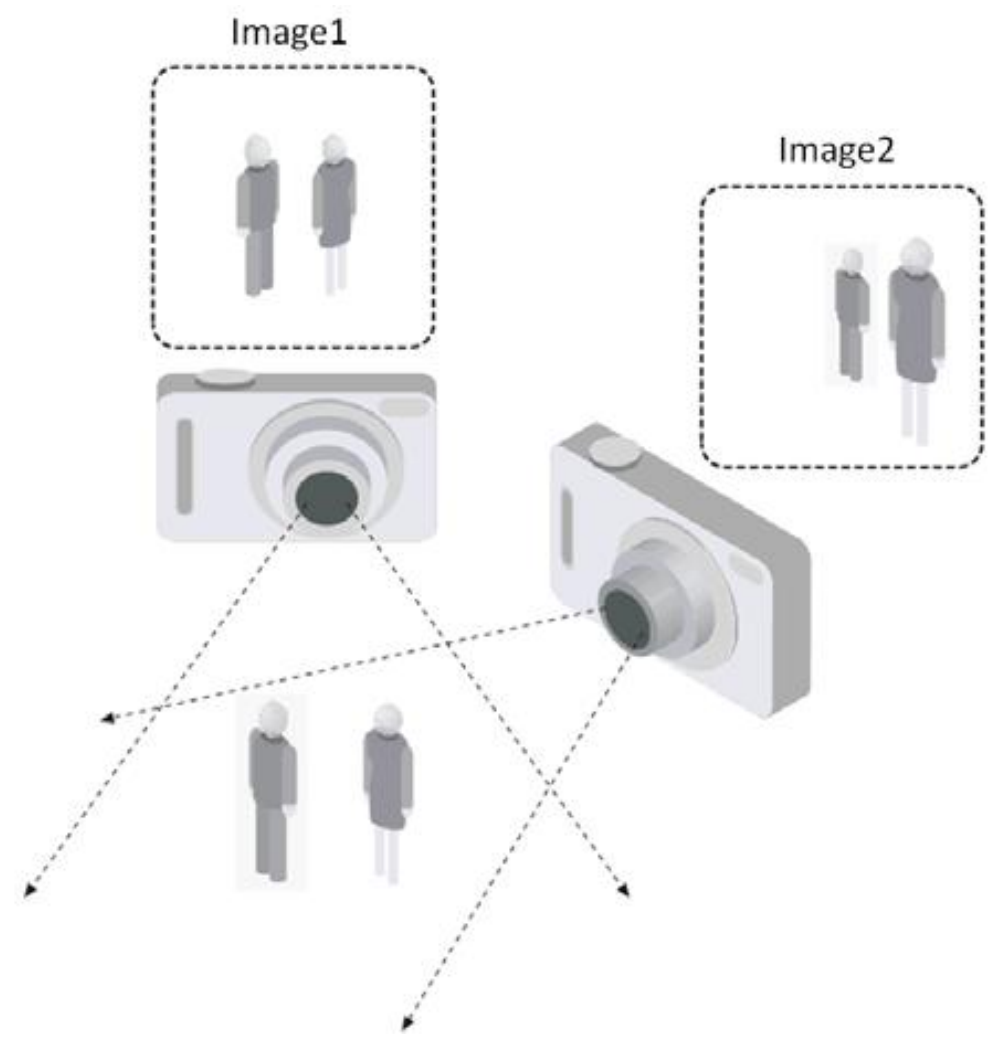

Figure 4: Stereo Cameras [2]

\section{Epipolar Geometry}

Epipolar Geometry is the basic geometry of stereo vision systems. It narrows down the search plan of the 3D scene feature correspondence. In particular, it limits the search plan for the corresponding feature to the epipolar lines. These lines are obtained by the two epipole points. An illustrative example of both epipoles and their correspondent epipolar lines is provided in Figure 5. In this example, $P_{l}$ and $P_{r}$ are the feature projections, and $e_{l}$ and $e_{r}$ are the epipoles. Using these projections and epipoles, the epipolar lines $P_{l} e_{l}$ and $P_{r} e_{r}$ are generated.

However, before the usage of the epipolar geometry, the stereo cameras orientation parameters need to be identified. These parameters represent the essential geometry of the stereo vision setup, i.e., essential matrix and fundamental matrix. 


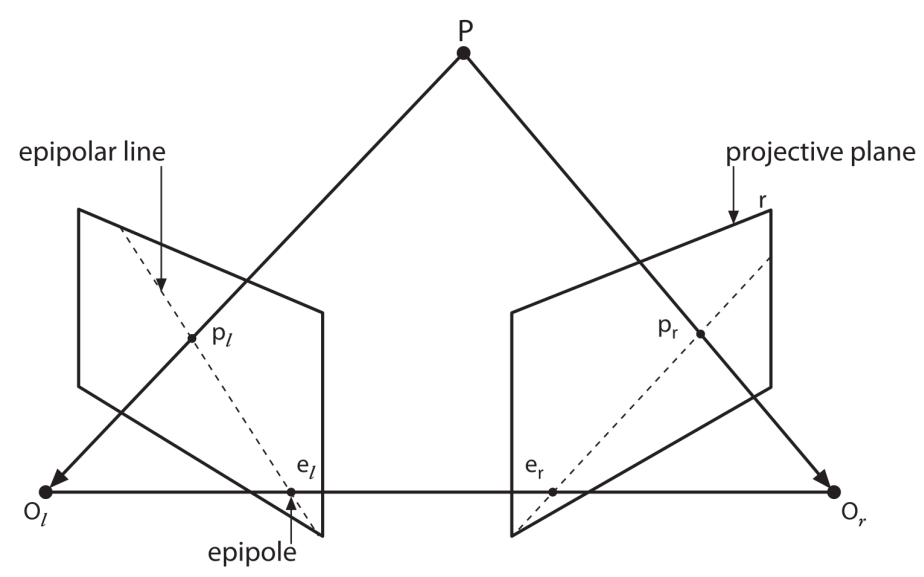

Figure 5: Epipolar Geometry [2]

\section{Essential Matrix}

The essential matrix, $E$, is the matrix that represents the geometric transformation between the two cameras coordinate systems in the physical world. The essential matrix is composed of a rotational matrix, $R$, and a translational vector, $T$, as shown in Figure 6. Note that the suffix $r$ and suffix $l$ are used to denote the right and the left image frames, respectively. The essential matrix, $E$, that maps the feature perspective $P_{l}$ to the feature perspective $P_{r}$ can be derived from the Euler relations $P_{l}=R_{l} P+T_{l}$ and $P_{r}=R_{r} P+T_{r}$. This essential matrix was shown in [2] to be used as

$$
P_{r}=E P_{l}
$$

where

$$
E=R S=R\left[\begin{array}{ccc}
0 & -T_{z} & T_{y} \\
T_{z} & 0 & -T_{x} \\
T_{y} & T_{x} & 0
\end{array}\right]
$$




$$
\begin{gathered}
T=\left[\begin{array}{l}
T_{x} \\
T_{y} \\
T_{z}
\end{array}\right], \\
R=R_{r}\left(R_{l}\right)^{T}, \\
T=T_{r}-R T_{l} .
\end{gathered}
$$

\section{Fundamental Matrix}

The provided essential matrix defines the geometric transformation of the stereo vision system. However, this transformation deals with a perfectly calibrated camera model. In order to overcome this drawback, the so called fundamental matrix $F$ can be used. This matrix represents a more general representation of the epipolar constraint that includes the cameras' intrinsic parameters. The fundamental matrix is defined by the relation $q=M P$, where $M$ represents the camera intrinsic parameters. Using this definition, the relation between $E$ and $F$ can be given as follows

$$
F=\left(M_{r}^{-} 1\right)^{T} E M_{l}^{-} 1
$$

Accordingly, the epipolar constraint would be

$$
q_{r}^{T} F q_{l}=0
$$

In essence, the essential matrix uses the physical coordinates, while the fundamental matrix uses the pixels coordinates. 


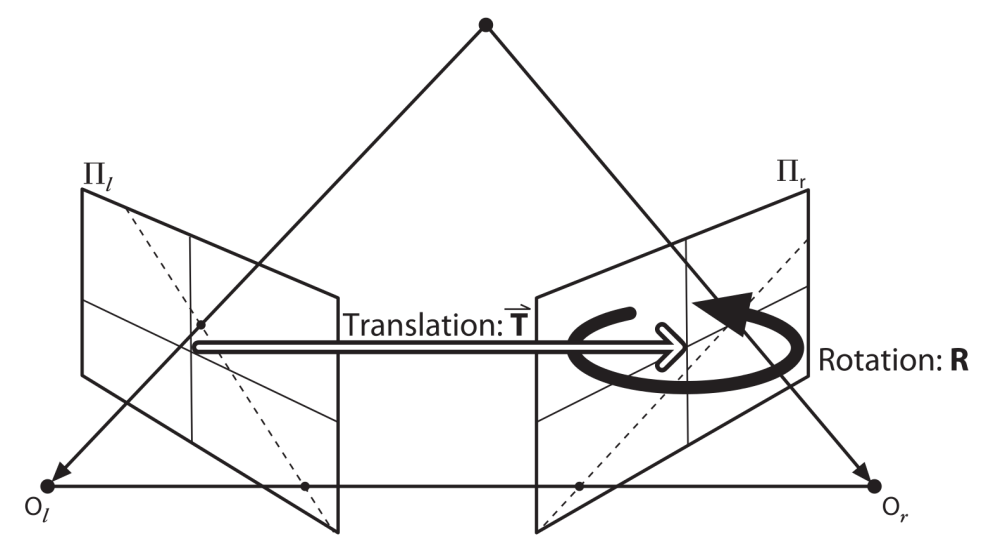

Figure 6: Essential Geometry [2]

\section{Calibration and Rectification}

As discussed hereinabove, stereo imaging systems can never have perfectly aligned image frames. To mitigate this drawback, two processes must be applied on stereo images, i.e., calibration and rectification. The objective of stereo cameras calibration is to identify the fundamental matrix of the stereo vision system that is used to map the observed feature correspondences. After calibrating the stereo images, another process is applied which is rectification. This process is used to enhance the stereo matching and correspondence, and simplify the computation of stereo disparity by aligning the rows of the two perspectives. To demonstrate the importance of the calibration and rectification processes, an illustrative example is depicted in Figure 7.

In this figure, the actual calibration and rectification processes starts with the corners detection in both perspectives as shown in (a). Process (b) uses the coordinates of the found corners to calibrate and undistort the raw images. Then, the actual coordinates are rectified by aligning the rows of the correspondent image pixels as shown in process (c). After the rectification, the perspectives are cropped to eliminate the undefined image parts. 


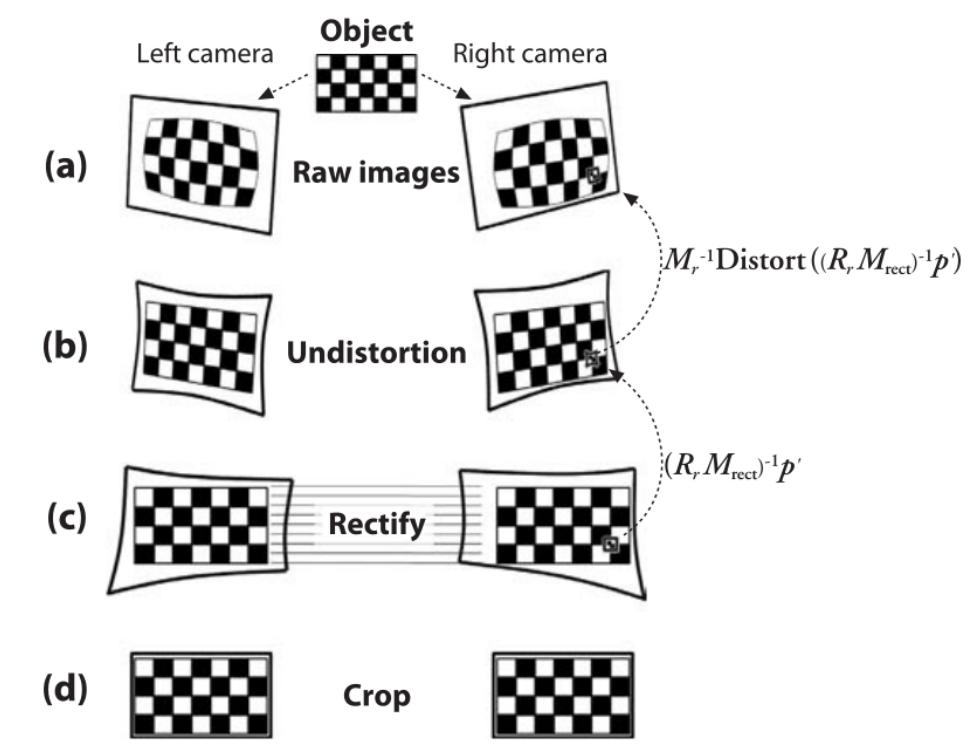

Figure 7: Calibration And Rectification [2]

\section{$2.4 \quad$ Visual Odometry}

Another approach for tracking robot motion is visual odometry. Such approach is used as an alternative to conventional motor odometry to define the robot motion and to overcome wheel slipping errors. A visual odometry system might have either a single or stereo cameras. These cameras are used to track the environment features by using what is called optical flow. The optical flow of the dynamic 3D scene is used to estimate the accumulative position and orientation of the robot. Therefore, such systems are open-loop systems that are subject to accumulative errors. 


\section{Chapter 3}

\section{Literature Review}

\subsection{Introduction}

There are different types of lenses used for capturing 2D images of the 3D environments. Conventional perspective lenses have single projection. Such lenses, have an average horizontal field of view of $60^{\circ}$. On the other hand, there are wide field of view lenses that can capture up to a full $360^{\circ}$ image. Such lenses are called panoramic or omni-directional lenses. In all cases, conventional, panoramic, and omni-directional lenses are mounted on a regular 2-D camera sensor with finite number of pixels. Panoramic vision systems have benefits and drawbacks when used in mobile robot applications. Such systems capture circular images with a wide field of view, i.e, cylindrical field of view. However, the cylindrical image projection on the 2D camera plane causes a loss of image information due to pixels size and location. In other words, the cylindrical field of view has a direct effect on the image quality. Despite this lower quality, the large image field of view has a great benefit for the mobile robot mapping process. In conclusion, when using panoramic images instead of conventional lenses, there is a trade-off between the field view and image quality. 


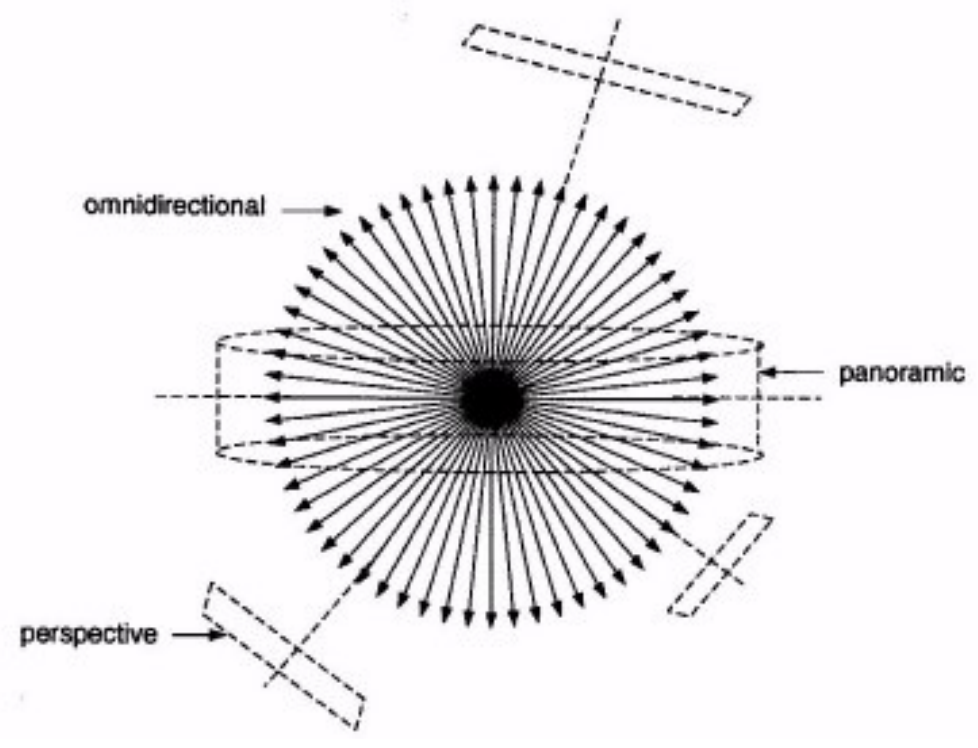

Figure 8: Panoramic Images [10]

\subsection{Panoramic and Omni-directional Lenses}

There are different models of $360^{\circ}$ lenses used. One example is the omni-directional lenses that have an extremely wide field of view of up to a $360^{\circ} \times 210^{\circ}$. Another example is the panoramic lenses that have a cylindrical $360^{\circ}$ field of view with a limited vertical range of view. Panoramic lenses are mostly catadioptric lenses while omnidirectional lenses are mostly fish-eye lenses. The catadioptric lenses have reflections and refractions, but the fish-eye lenses have refractions only. Both types of lenses are discussed next.

\subsubsection{Fish-Eye Lenses}

A fish-eye lens is composed of a combination of convex and concave lenses as shown in Figure 9. The multiple levels of refraction allow the fish-eye lens to capture a wide field of view. However, such lens construction introduces a design complexity along with increasing image distortions. 


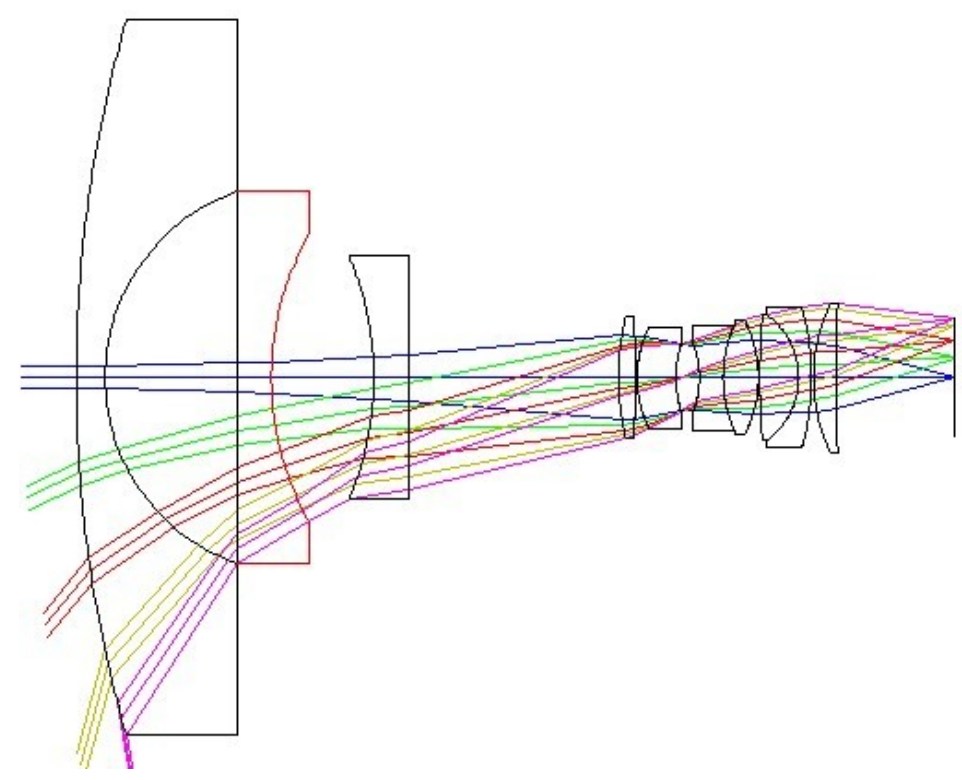

Figure 9: Jollystar Fisheye Lens

\subsubsection{Catadioptric Lenses}

Catadioptric optical system is based on a combination of optical reflections and

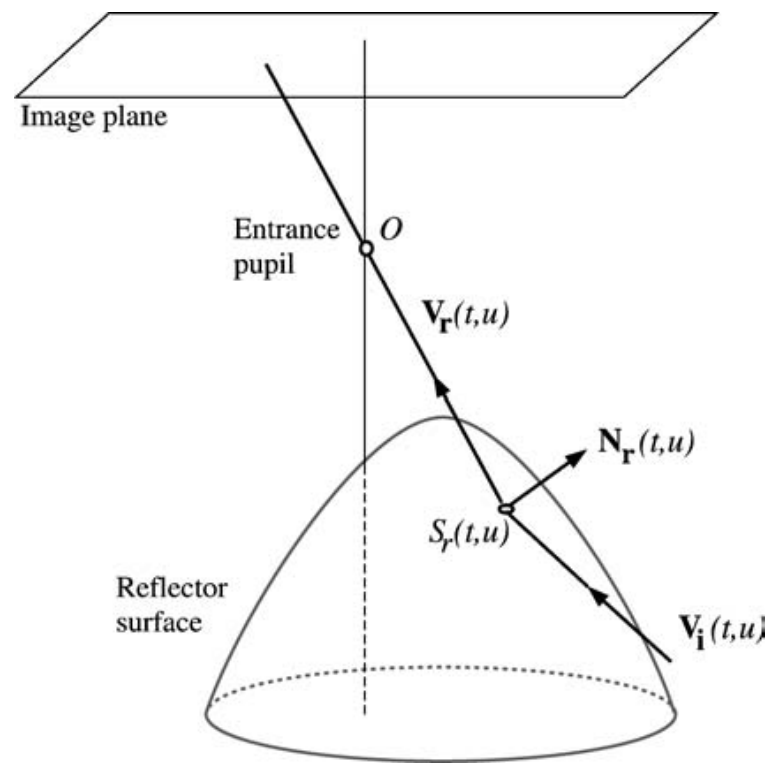

Figure 10: Single Mirror Panoramic Lens [11] 


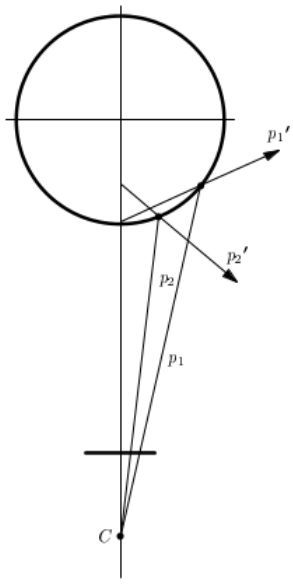

(a) Spherical mirror and a perspective camera. The reflected optical rays do not intersect in a unique point and the sensor suffers from spherical aberration.

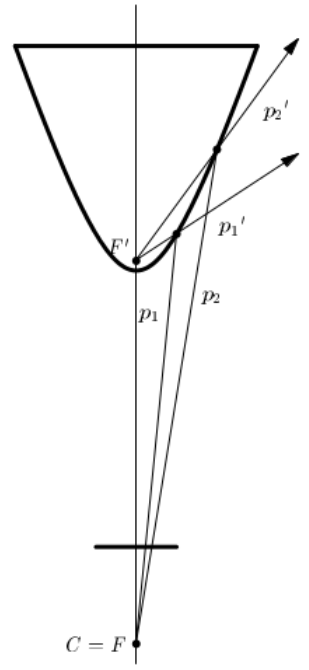

(b) Hyperbolic mirror and a perspective camera. The reflected optical rays intersect in the focus of the hyperboloid.

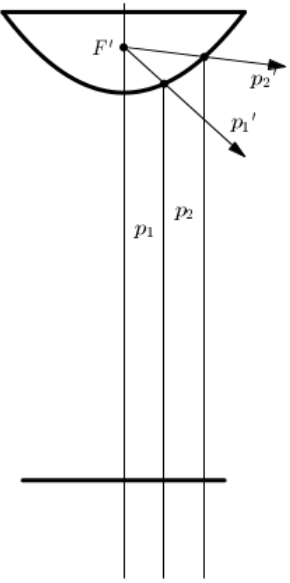

(c) Parabolic mirror and a perspective camera. The reflected optical ravs intersect in the focus of the paraboloid when orthographic projection is assumed.

Figure 11: Panoramic Mirrors [12]

refractions. In particular, single-viewpoint catadioptric lenses have conic shape mirrors. Such mirrors reflect the incident light rays towards a desired location as shown in Figure 10. These mirrors might have different structures, i.e., hyperboloid, spheroid, and/or paraboloid. Each of these structures has advantages and disadvantages as discussed below.

\section{- Hyperbolic Mirrors}

Hyperboloid mirrors constitute a category of mirrors that are used in catadioptric lenses. This shape of mirrors introduces a design complexity because it uses a specially designed aspheric mirror that is expensive and difficult to make. Moreover, the calibration process is more complex and difficult because the viewpoint of such camera must be mounted accurately at a precise location and orientation that depends on the hyperboloid design. Despite its complexity, hyperbolic mirros have compact size with a wide field of view as shown in 


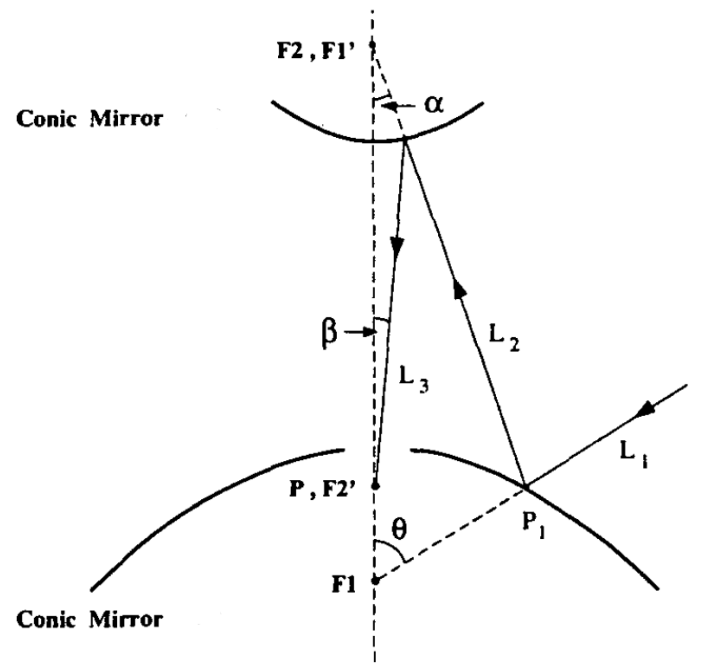

Figure 12: Folded Panoramic Lens [9]

Figure 11.

\section{- Spherical Mirrors}

Spheroid mirrors are another category of mirrors used in catadioptric lenses. Such lens structure does not have a single viewpoint and provides a good resolution at high elevations up to 90 degrees. However, below the horizontal elevation, the resolution significantly deteriorates.

\section{- Parabolic}

Paraboloid mirrors are easier to use in Catadipotric lenses. This type of lenses reflects parallel rays of light along the lens optical axis. In particular, those light rays can be captured from any distance as long as the optical axis of both, the camera and the lens, are aligned. However, a major drawback of using such mirrors is the size. In particular, such systems require the camera lens to be as wide as the parabolic mirror. 


\subsection{Folded Panoramic Lenses}

Folded panoramic lenses are another types of catadioptric lenses. They are composed of multiple mirrors and/or lenses as shown in Figure 12. Such type of lenses is compact in size and better in performance. In addition, they provide a customizable field of view and thus can be designed to fulfill specific tasks or requirements.

\subsection{Omni-directional Camera Calibration}

The calibration process of stereo omni-directional cameras is used to define the intrinsic and extrinsic camera parameters. The intrinsic camera parameters includes the distortion parameters of the used conventional cameras plus the omni-directional lens optical characteristics. Moreover, the extrinsic camera parameters are the parameters representing the relative $3 \mathrm{D}$ position and orientation of the stereo panoramic cameras. In comparison to the conventional camera calibration, omni-directional cameras have an additional calibration step for defining the optical characteristics of the used panoramic lenses.

\subsubsection{Single Camera Calibration}

The calibration approaches for omni-directional cameras can fall into two categories. The first category is based on calibrating omni-directional images in the presence of a predefined scene patterns, while the second is based on the usage of unknown scenes with feature correspondences and epipolar constraints.

\section{First Category}

One example on the calibration approaches of this category is the work presented in [27]. In this work, the authors used a Taylor series expansion to define the intrinsic 
camera projection model. To do so, they used different perspectives of a calibration pattern, i.e., different views of the calibration pattern. In contrast, the authors of [3] used predefined 3D coordinates of the calibration board corners and their correspondent $2 \mathrm{D}$ representation on the captured image to define the optical transformation of the omni-directional lens.

\section{Second Category}

One example of this category used visual odometry and ego-motion that depends on camera motion [29]. In particular, they used a camera that is mounted on a moving robot. Another method is the work presented in [30]. In this work, the authors developed a calibration approach that is dependent on the epipolar constraints of the camera lens, and the linearization of the fish-eye lens model.

\subsubsection{Stereo Calibration}

The calibration of stereo omni-directional cameras is highly dependent on the underlying stereo setup. In particular, there exists two different setups for multiviewpoint stereo systems. One is co-axial while the other is side-to-side. However, in practical applications, the co-axial setup of stereo omni-directional cameras is commonly used since it allows for easier rectification and feature matching. This is because, co-axial omni-directional images are rectified on a column by column basis. However, this format requires a relatively large height allowance for the robotic platform and thus might not be readily implementable in some applications, e.g., indoor robotic vacuum cleaners. Unlike the previously discussed stereo setups which have two cameras, there exists another stereo setup that uses a combination of a camera and a sensor, e.g., a laser sensor. Next, examples of the stereo calibration approaches are provided for these three setups. 


\section{Accuracy Enhancement of Omni-directional Camera Calibration}

In the provided work in [31], the authors introduced a calibration approach for omni-directional cameras. In their approach they used an omni-directional movable camera along a certain plane to resemble a side-to-side omni-directional setup. This camera is observing a pre-defined 3D calibration pattern, i.e., trihedron. However, this model is over constrained and needs special conditions to be applicable. In particular, it requires a special setup for the camera calibration process.

\section{Co-axial Stereo Calibration}

One approach for the calibration of co-axial cameras was proposed in [32]. In this work, the calibration was performed by using a calibration pattern along with the epipolar geometry constraints. In [33], a calibration method was proposed for a specially designed co-axial stereo setup. In particular, in this setup they used a single camera that was pointed towards two omni-directional lenses. To calibrate this model, the authors used a pre-defined calibration pattern. However, this special coaxial setup has a major drawback. In particular, it has a limited stereo baseline, i.e., the distance between the two lenses. This small stereo baseline reduces the system ability to estimate the distance of far objects.

\section{Calibration of an Omni-directional Camera and a 2D Laser Rangefinder}

In [34], the authors introduced an approach for calibrating a combination of a laser scanner and an omni-directional camera. This approach used a pre-defined 3D calibration board. In this approach, the calibration board features are matched using the discontinuity in the depth of the 3D calibration board. In particular, since laser scanners can only detect the depth, the authors introduced a special calibration board that contains different depths. 


\subsection{Mobile Robots Positioning}

Mobile robot positioning requires accurate depth estimation of environment features. There exists different approaches in the literature for environment feature positioning using omni-directional cameras. One approach is based on tracking the optical fields of a single camera. Two examples of this approach are visual odometry and sensor fusion, in which the outputs of two sensor types are integrated, e.g., a camera and tackometer or laser range finder. Another approach is based on using conventional or omni-directional stereo vision systems that provide different perspective of a 3D scene and subsequently allows depth estimation. In the following sections, examples of the above positioning approaches are provided.

\section{Virtual Compass}

In [35], the authors introduced a technique called virtual compass that utilizes a single omni-directional camera model. This camera was pointed towards the ceiling and was used to detect three parallel features of the 3D scene. These features were then used to estimate the rotation and the translation of the mobile platform. However, this technique does not work if there are not enough parallel features in the ceiling.

\section{Color Matched Features}

In [36], a positioning technique for side-to-side stereo omni-directional vision system was proposed. This technique is based on the extraction of colored landmarks in a controlled environment. However, the practicality of this positioning approach is questionable since it requires an ideal workspace with specific lighting conditions. 


\section{Dynamic Objects Detection Using one Omni-directional Camera}

In [37], the authors had introduced a method for dynamic objects positioning and tracking using the optical flow observed by a mobile robot. In this method, the flow vectors were clustered to determine the dynamic and static objects on the observed circular omni-directional scene. In addition, these vectors were also used to define the mobile robot center of gravity, which enables the robot to successfully follow the dynamic object.

\section{Mobile Robots Head Estimation Using Vanish Points}

In $[38,39]$, a navigation approach was developed using a single omni-directional camera. This approach was based on the robot positioning within parallel features, e.g., road lanes. To do so, the robot followed the vanish points (VPs) of the observed parallel features.

To detect the vanish points, the authors proposed the methods in [41,42]. In [41], the vanish points were detected using the longest observed line segments. It is noted that, due to the panoramic lens structure, these line segments were observed as curves on the image plane and thus their equations must be defined. To do so, the authors used Lagrange coefficients. Subsequently, the intersecting points of these curves were estimated and defined as vanish points. Finally, to enhance the estimation quality, the authors eliminated the noise by clustering the data using the Density-Based Spatial Clustering of Applications with Noise (DBSCAN) algorithm proposed in [40]. In [42], the authors developed another method for the detection of the VPs. This method identified the image line segments using canny edge detector [43]. Then, Random Sample Consensus (RANSAC) curve fitting was applied to define the curves and estimate the vanish points. Finally DBSCAN was used to cluster the extracted data to enhance the estimation quality of the VPs. 


\section{Biologically Inspired Navigation Using Landmark-Tree Maps}

In [44], the authors introduced a biologically inspired navigation method. This method used environment features and landmarks to localize the mobile robot during operation. These features were memorized in a tree data structure that forms a hierarchy, i.e., Landmark-Tree (LT) map, according to their distance from the robot. Using this map, the robot could implicitly identify its path without requiring direct depth measurement. The advantage of this LT map is that it reduces the memory usage of the mobile robot in long path operation and thus enables scalability. However, this method does not provide absolute positioning because it does not have metric distance information.

\section{Dynamic Objects Detection Using Ego-Motion}

In [45], the authors proposed a method for positioning the moving objects in dynamic environment. To do this, the method detects the optical flow from consecutive frames by Kanade-Lucas-Tomasi (KLT) optical flow tracker. Then, moving objects are clustered by Ego-Motion compensation to eliminate the optical flow caused by robot movement. However, in this work, this method was used for human detection by applying histogram vertical projection with specific threshold to define a moving human.

\section{Navigation of an Automatic Guided Vehicle Utilising Omnivision}

In [46], a technique for robot navigation using a single omni-directional camera was introduced. The authors used an area of vision interest to define the direction of robot motion. The proposed area of vision interest was the area with straightest lines in the perspective of robot motion. This robot was developed to navigate within indoor environments while following observed signs in the direction of robot motion. 


\section{Omni-vision Robot Localization Using Pattern Match System}

In [47], the author presented a method for robot localization in a pre-defined work space, i.e., a soccer playground. In this method, the robot used an omni-directional vision sensor along with a gyroscopic sensor for localization. In particular, the pattern of the playground is defined to be at a reference angle. This robot localization method used the gyroscopic sensor to define its orientation and the panoramic vision sensor to observe the patterns of the playground. Using this information, the robot then implicitly defined its location and orientation within the work space. However, this approach is applicable for predefined and controlled environments only.

\section{Intuitive 3D Maps for MAV Terrain Exploration and Obstacle Avoidance}

In [48], textured 3D mesh points were introduced to be used for terrain exploration and surface reconstruction. In this work, the authors showed the use of the 3D mesh in localizing and controlling the robot while doing the surface reconstruction simultaneously at minimal extra cost.

\section{Monocular Visual Odometry and Pixel Intensities}

In [49], a fast visual odometry approach was proposed. In particular, the authors used pixel intensities, which resulted in sub-pixel precision at high frame rate. They, also, used a probabilistic mapping approach to estimate the 3D points. These resulted in fewer outliers and more reliable points estimation. This approach was applied for conventional stereo vision systems.

\section{Stereo Omni-directional Vision System for Teleoperation of Mobile Robots}

In [50], the author introduced an approach for stereo wide-angle vision systems. 
This system was composed of a wide-angle camera pointed forward and another horizontally co-axial catadioptric lens. This unconventional setup enabled its application in low profile robots.

\section{Optimal Design and Placement of Omni-Cameras}

To assess depth measurement precision, the authors in [51] developed formulas describing the 3D depth estimation error propagation. In addition, they introduced an algorithm for determining the optimum stereo configuration. To do so, they used raw omni-directional images. The proposed algorithm was applied for human-computer interaction framework.

\section{Moving Objects Detection Using Omni-directional Camera and Wheels Odometry}

In [52], a method for positioning dynamic objects in an observed scene was provided. This method fused the visual odometry captured by an omni-directional camera and wheels odometry. In particular, they used the wheels odometry to eliminate the optical flow of the static scene objects. However, this method is subject to errors due to the wheels slipping.

\section{1-Point-RANSAC by Exploiting Non-holonomic Constraints}

For wheeled vehicles, the authors in [53] provided a method for using 1 point RANSAC structure from motion with a single omni-directional camera mounted over a rover. This was done by exploiting the non-holonomic constraints of the wheeled rover motion. However, the proposed method is subject to accumulative odometry errors. 


\section{Active Vision}

Another technique for mobile robot positioning was proposed in [54]. This technique was based on active vision that is independent of environment textures. In particular, it worked by projecting a laser pattern on the environment. These projections were then detected by the camera and used to define the depth of environment features.

\section{Global Path Planning with Ceiling Mounted Camera}

In [55], a technique for mobile robot positioning was developed. Unlike the previous techniques, the omni-directional camera in this technique was mounted on the ceiling rather than on the platform of the robot. However, this approach is not desirable because mobile robots are intended to have the ability to work in any given environment without prior preparation.

\section{Omni-directional Multi Perspective System}

In [56], a positioning approach using omni vision system was proposed. This approach used multiple projection cameras on a spherical plan. The usage of these perspectives enabled depth estimation of environment features. However, In this paper, the approach was applied in a surveillance applications that used 44 static cameras.

\subsection{The Proposed Approach}

\subsubsection{Calibration}

Unlike previous work, the proposed approach calibrates the unwrapped panoramic images. In particular, the spherical geometry is used with a folded lens model that 
have constant vertical and horizontal angular steps. By exploiting these constraints, the calibration approach targets obtaining two variables, i.e., the observed horizon of the panoramic field of view and the vertical field of view with its correspondent angular step size. In order to define the horizon of the provided perspective, the vanish points of two observed parallel features in the unwrapped image frame need to be defined. In particular, a chessboard with extra lines added at its far ends is used to allow the observation of two physically parallel curves at higher slope. This results in enhancing the accuracy of the calibration process and reducing the correspondent errors. However, this approach requires the parallel features to be parallel to the horizon of the given perspective. Therefore, this approach imposes a constraint on the orientation of the calibration board. In particular, it requires the calibration board to be parallel to the horizon of the panoramic lens. This condition is satisfied when each column of physical chessboard corners is checked to be sharing a specific column of pixels in the image frame. In addition, the vertical angular step size is defined by using the curve equation of the observed parallel features. It is noted that the two calibration variables are defined in pixel coordinates.

\subsubsection{Stereo Correspondence and Positioning}

Similar to previous work, a predefined calibration pattern is used to estimate the relation between the two panoramic perspectives. In particular, it helps in defining the epipolar geometry that allows for more robust features matching. Unlike the previous work, an epipolar geometry model for the unwrapped side-to-side stereo omnidirectional cameras is proposed. In particular, this model is based on the spherical geometry of the given panoramic perspectives and is proven to generate a sinusoidal epipolar curve. In order to evaluate the quality of the proposed approach, depth estimation analysis is performed for different setup dimensions and different image qualities. This analysis proved the feasibility of this approach for the proposed robotic 
vacuuming application in indoor environments, i.e., relatively small size environment. However, more research is required to enable autonomous feature matching along the provided epipolar curve. 


\section{Chapter 4}

\section{Panoramic Camera Calibration}

\subsection{Panoramic Image Unwrapping}

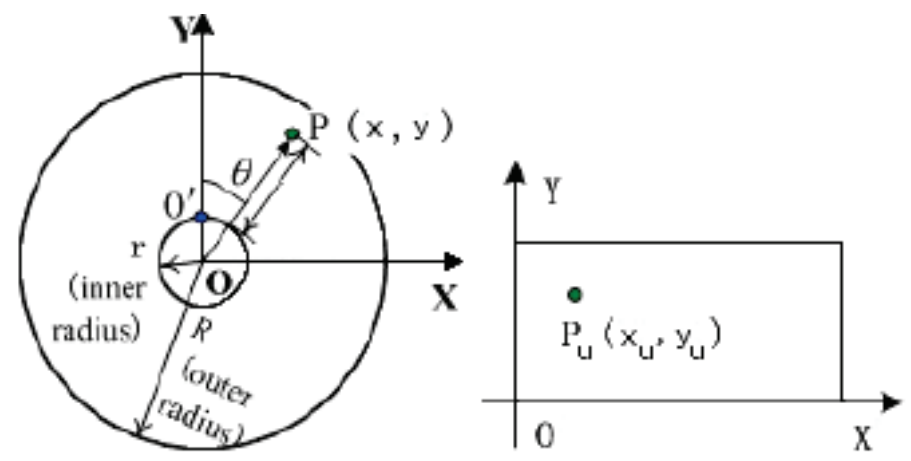

(a) circularity panorama

b) cylinder panorama

Figure 13: Image Unwrapping [13]

Image unwrapping is the process of transforming the circular image frame to an unwrapped cylindrical image frame. This process is a primary prerequisite for the proposed calibration approach of panoramic cameras. This is because the unwrapped images provide simpler system visualization. An illustrative example of the image unwrapping process is depicted in Figure 13 and the details of the unwrapping process is provided in the following sections. 


\subsubsection{Circular Panoramic Image Parameters}

Panoramic lenses capture a cylindrical image of the real world. The projection of this cylindrical image is a circle on the image plane. One example of the output of the panoramic lens and its circular projection is provided in Figure 13. To perform the unwrapping process, it's essential to identify the circle parameters, i.e., the outer radius, inner radius, and the circular image center. These parameters are illustrated graphically in Figure 13(a). One approach for identifying these parameters is the Hough-circle transform, which is described next.

\section{Hough Transform}

Hough transform is a commonly used approach for the detection of shapes in a given image. To do so, Hough transform uses edges, i.e., pixels with high contrast, as the raw input data. In particular, Hough transform uses the required shape's parametric equations to define the existence of this shape and and define its parameters. This is done by performing exhaustive search over all found edges in the image for the satisfaction of the parametric equation of the required shape.

One example of the Hough transforms is the Hough line. This transformation is used to find all possible lines within a given image by utilizing the line parametric equation

$$
r(\theta)=x \cos \theta+y \sin \theta
$$

Where $(x, y)$ are the cartesian coordinates of a pixel in a given image, $\rho$ is the length of the normal from the origin to a line containing the point $(x, y)$, and $\theta$ is the angular orientation of the normal, $\rho$. For any given straight line, there is only one unique solution of $(\rho, \theta)$ that satisfies the parametric equation as shown in Figure 14. Using this fact, the presence of line segments can be detected. This is done by inspecting all found edges within an image. If any of these edges satisfy the 

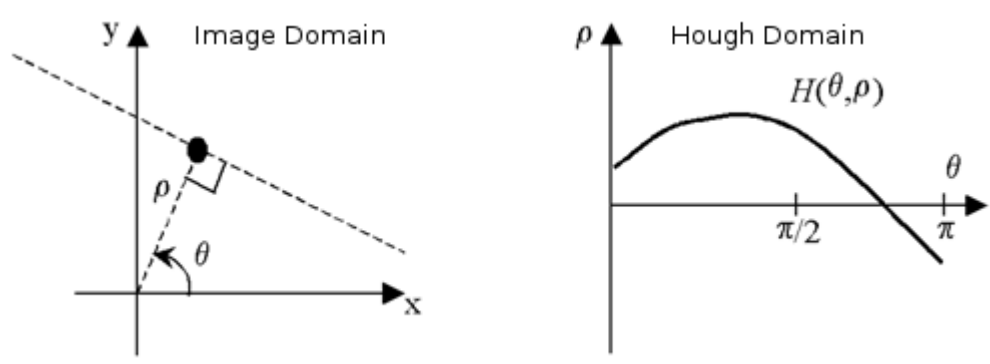

Figure 14: Hough Line Transform ${ }^{1}$

line parametric equation presented above, it will be identified as a straight line.

Another example is the Hough circle transform. This transform can be used for the identification of circles within an image. Unlike the Hough line, Hough circle detects circles by identifying 3 parameters. Theses parameters are the coordinates of the found circle's center, i.e., $\left(x_{c}, y_{c}\right)$, and the radius of this circle, i.e., $r$. This is done by using the circle parametric equation

$$
\left[\begin{array}{l}
x \\
y
\end{array}\right]=\left[\begin{array}{c}
x_{c}+r \times \cos \theta \\
y_{c}+r \times \cos \theta
\end{array}\right]
$$

where $\theta$ is an iterative angle between $0^{\circ}$ and $360^{\circ}$. It is noted that the three circle parameters, i.e., $\left(x_{c}, y_{c}\right)$ and $r$, are unique for any given circle.

Following the same principles of the Hough line transform, the Hough circle can detect the presence of circles within an image. This is done by inspecting all found edges within an image for the satisfaction of the circle parametric equation. In addition, the computational efficiency of the hough-circle transform can be increased by setting a range of radius $r$ that prevents from the detection of circle with undesired sizes and reduces the computational cost.

\footnotetext{
${ }^{1}$ http: //www.exelisvis.com/docs/HOUGH.html
} 


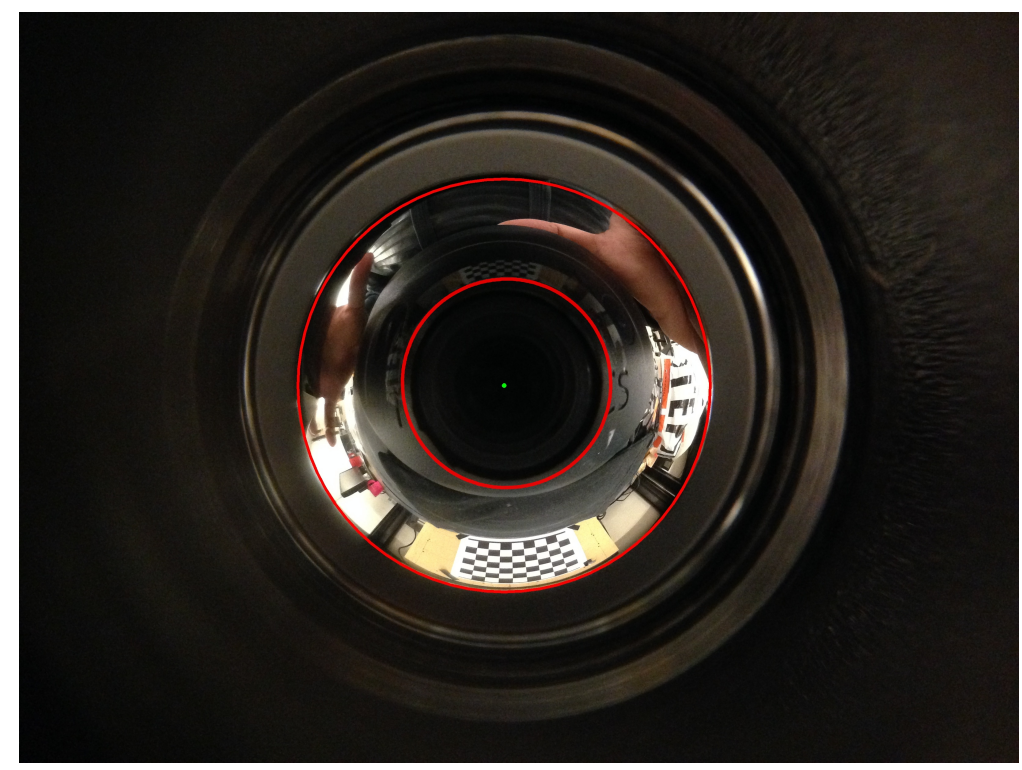

Figure 15: Application of Hough Circle Transform

\subsubsection{Image Unwrapping}

As discussed above, the raw output image of a panoramic lens is a circular image. When this image is unwrapped, each pixel will correspond to a specific azimuth and altitude angle. In other words, each pixel of the unwrapped image can be linearly mapped to a specific observation of the real world as shown in Figure 16.

It is noted that, due to the structure of the panoramic lens, the output raw circular image has a fragment that does not correspond to any physical world observation. This is a result of the fact of capturing a cylindrical perspective of the physical world on a rectangular image sensor. Thus, to save the processing power, this part has to be ignored during the image unwrapping process. This can be done by defining the minimum and maximum circles radius, $R_{\min }$ and $R_{\max }$. Moreover, in this work, the inner radius $R_{\min }$ and the outer radius $R_{\max }$ are expressed in pixel coordinates.

The process of image unwrapping involves two steps. First, the minimum and maximum circumferences of the circular image are defined. These circumferences are used to identify the length of the circular slices of the observed circular image. 


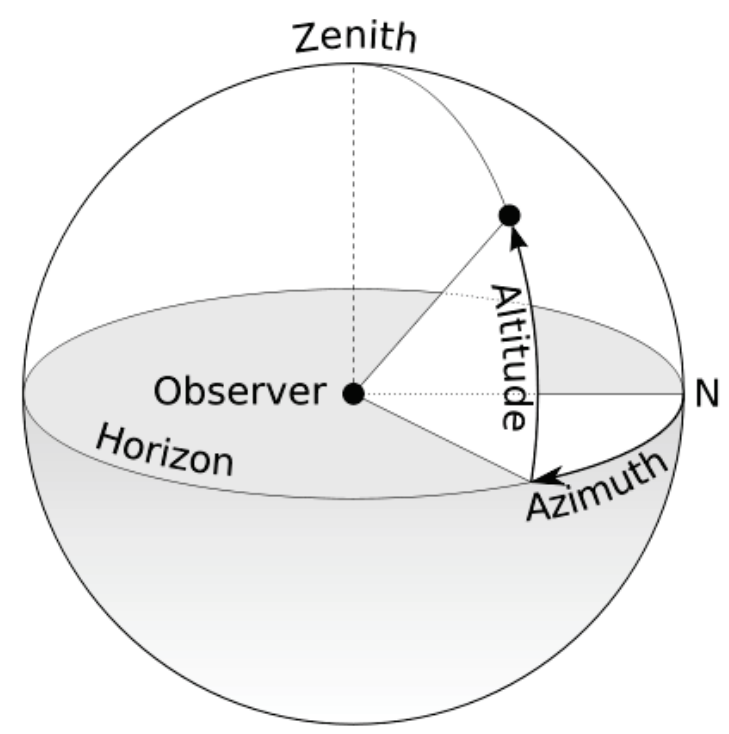

Figure 16: Circular Geometry of Omni-directional Images ${ }^{2}$

Second, each of these slices is mapped to a row in the rectangular unwrapped image frame. However, due to the discrepancies in the lengths of these circumferences, the number of pixels in each circular slice is different. Therefore, a problem arises when these circular slices are mapped to the rows of pixels in the unwrapped image. In particular, all circular slices except the one corresponding to the maximum radius will need to be stretched if the maximum circumference is chosen as a reference for the unwrapped image length, or all slices except the one corresponding to the minimum radius will need to be compressed if the minimum circumference is chosen as a reference for the unwrapped image length. Since the camera sensors have a 2-D array of discrete pixels, the stretching and compression processes will be translated into pixels repetition and skipping, respectively. Therefore, this has a direct effect on the unwrapped image quality. In particular, if the maximum circumference is chosen as the unwrapped image length, valuable image definitions will be lost from the minimum circumference end. Similarly, if the minimum circumference is chosen

\footnotetext{
${ }^{2}$ http://en.wikipedia.org/wiki/Azimuth\#mediaviewer/File:Azimuth-Altitude_ schematic.svg
} 
as unwrapped image length, the image definitions will be lost from the maximum circumference side. Subsequently, this limits the system ability to detect the image features. To mitigate this drawback, in this work, the average circumference is chosen to be the length of the unwrapped image. This reduces the maximum stretching and compression errors on both ends and, as a result, increases the unwrapped image quality. In this work, the average circumference is denoted by $K$, and evaluated it by

$$
K=2 \times \pi \times J
$$

where $J$ is the average radius and it's given by

$$
J=\frac{R_{\max }+R_{\min }}{2}
$$

Using the value of $K$, the pixels mapping between the circular raw image of the panoramic lens and the unwrapped rectangular image is given by

$$
\left[\begin{array}{l}
x_{u}^{i j} \\
y_{u}^{i j}
\end{array}\right]=\left[\begin{array}{l}
x_{c}+R_{j} \times \cos \theta_{i} \\
y_{c}+R_{j} \times \sin \theta_{i}
\end{array}\right],
$$

where $\left(x_{u}^{i j}, y_{u}^{i j}\right)$ are the coordinates of the unwrapped image pixel $P_{u}$ at row $i \in$ $\{1, \ldots, K\}$ and column $j \in\left\{0, \ldots,\left(R_{\max }-R_{\min }\right)\right\}$. The value of $R_{j}$ is equal to

$$
R_{j}=j+R_{\text {min }}
$$

and the value of $\theta_{i}$ is equal to

$$
\theta_{i}=360 \times \frac{i-1}{K}
$$




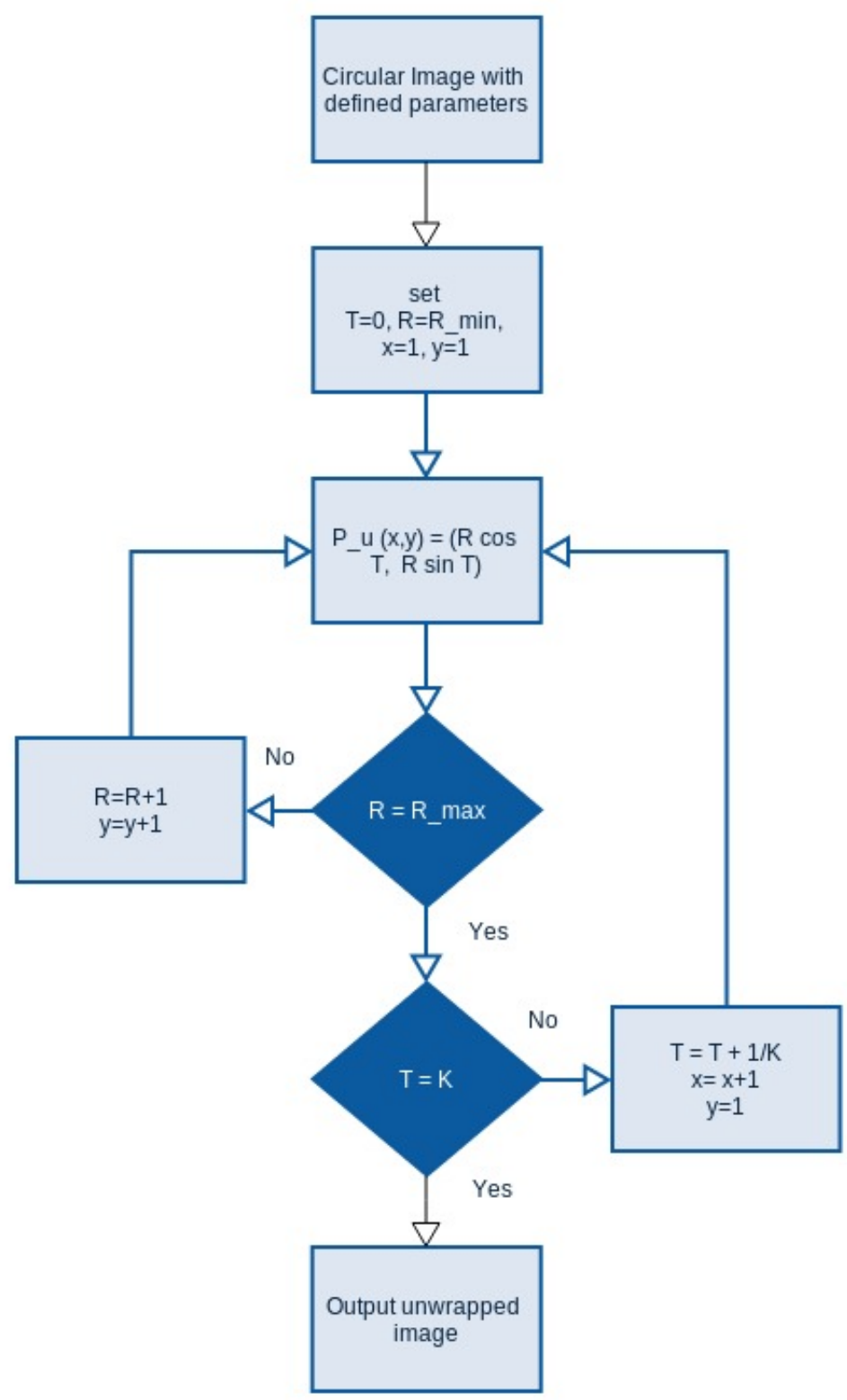

Figure 17: Image Unwrapping Flowchart

To further illustrate the unwrapping process, a flowchart of the proposed unwrapping process is provided in Figure 17.

\subsubsection{Experiments}

In this section, the performance of the image unwrapping approach is evaluated by 
experiments. In the following experiment, Hough circle transform is applied to detect the panoramic circular image parameters, i.e., $\left(x_{c}, y_{c}\right), R_{\max }$, and $R_{\min }$. The values of $R_{\max }$ and $R_{\min }$ of the circular image were found to be 571 and 289 , respectively. Subsequently, the values of $K, J$, and $\theta_{i}$ can be calculated by Equations (13), (14), and (17) as

$$
\begin{gathered}
K=2 \times \pi \times J=2701, \text { and } \\
J=\frac{571+289}{2}=430 \\
\theta_{i}=360 \times \frac{i-1}{2710}=0.1333 i
\end{gathered}
$$

In the provided setup, two identical cameras are used with identical panoramic lenses. Hence, both cameras have the same $R_{\max }$ and $R_{\min }$ values. However, due to the assembly imperfections, the coordinates of the of the two observed circular images are not identical. In particular, the center of the two circular images are found to be at $(1240,943)$ and $(1214,979)$.

By substituting in the unwrapping relation provided in Equation (15), the unwrapping function of the two cameras can be given by

$$
\begin{aligned}
& {\left[\begin{array}{l}
x_{u}^{i j} \\
y_{u}^{i j}
\end{array}\right]=\left[\begin{array}{c}
1240+(j+289) \times \cos (0.1333 i) \\
943+(j+289) \times \sin (0.1333 i)
\end{array}\right],} \\
& {\left[\begin{array}{l}
x_{u}^{i j} \\
y_{u}^{i j}
\end{array}\right]=\left[\begin{array}{c}
1214+(j+289) \times \cos (0.1333 i) \\
979+(j+289) \times \sin (0.1333 i)
\end{array}\right],}
\end{aligned}
$$

Finally, the raw circular images of the panoramic camera are provided in Figure 18 


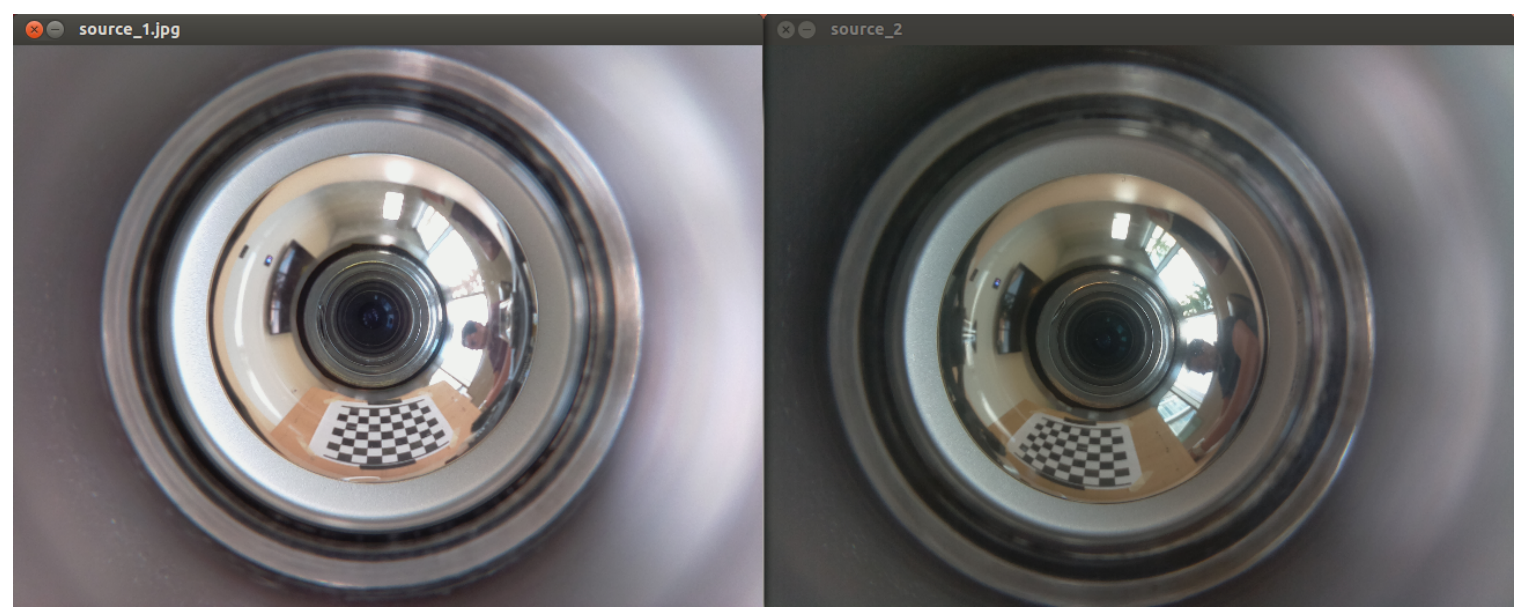

Figure 18: Source Images

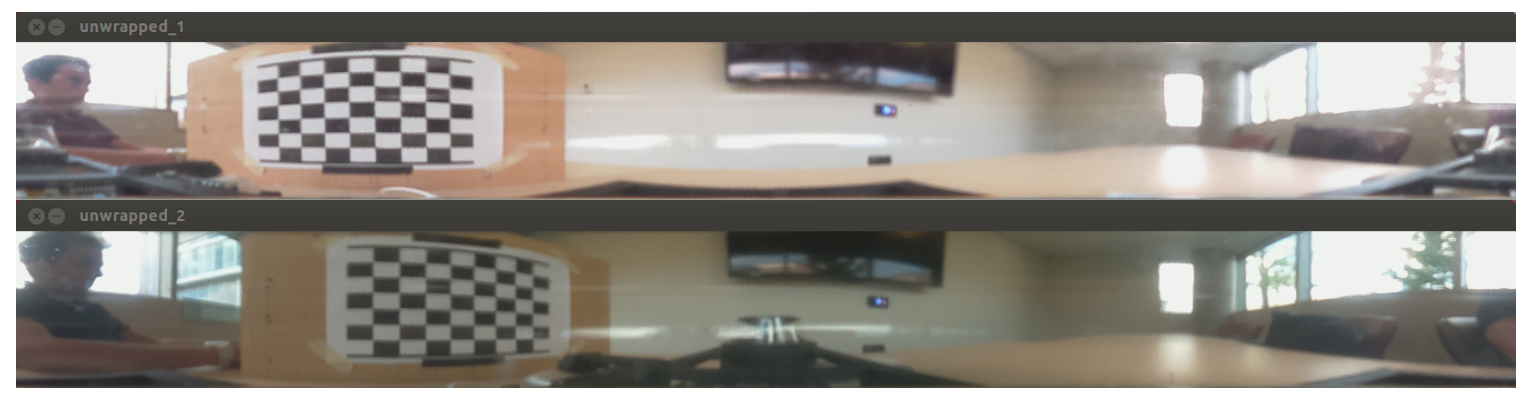

Figure 19: Unwrapped Images

and their correspondent unwrapped images obtained by the previous unwrapping simulation are provided in Figure 19.

\subsection{Geometric Model}

The geometric model of the vision systems illustrates how cameras capture light from their surrounding environment. In particular, the panoramic imaging system geometric model consists of the azimuth and altitude angular fields of view, where the field of view is defined as the maximum horizontal or vertical extent of an observable 3D scene that can be seen at any given moment.

In this work, the altitude and azimuth angles are denoted by $\gamma$ and $\theta$, respectively. 
In panoramic lenses, the azimuth angular step is constant along the panoramic view and the altitude angular step is dependent on the lens model. In addition, the horizontal field of view, i.e., azimuth angle of view, is known to have $360^{\circ}$ visual field, while the vertical field of view, i.e., altitude angle of view, can have different ranges of view based on the panoramic lens construction.

Since in this work the unwrapped images are considered to represent a cylindrical visual field, the horizontal straight lines in the physical 3D world will be observed as curves in the unwrapped images. In order to be able to manipulate the unwrapped panoramic images, the model that relates the horizontal straight lines with their correspondent curved perspectives in the unwrapped images is derived.

The curved perspectives of the observed straight features will form concave and convex curves in the upper and lower horizontal fields of view. This observations is essential since it ensures that there always exists a peak of an observed horizontal feature in the unwrapped image frames. This peak corresponds to the normal distance between the lens center of projection and the observed horizontal lines of the physical world.

Before describing the details of the geometric model, it is noted that the objective of this model is to relate the angles $\gamma, \gamma^{\prime}$ and $\theta$, where $\gamma$ is the altitude angle of the normal distance between the center of projection and the horizontal feature, i.e., the peak of the observed curve, $\gamma^{\prime}$ is the altitude angle of any given point along the observed curve, and $\theta$ is the azimuth angle between the projection of $\gamma$ and $\gamma^{\prime}$. Then, the relations between the physical world and its observed perspectives shown in Figure 20 are provided. These relations are given by

$$
\begin{gathered}
\cos \theta=\frac{Z^{\prime}}{Z}, \\
\tan \gamma^{\prime}=\frac{H}{Z^{\prime}}, \text { and }
\end{gathered}
$$




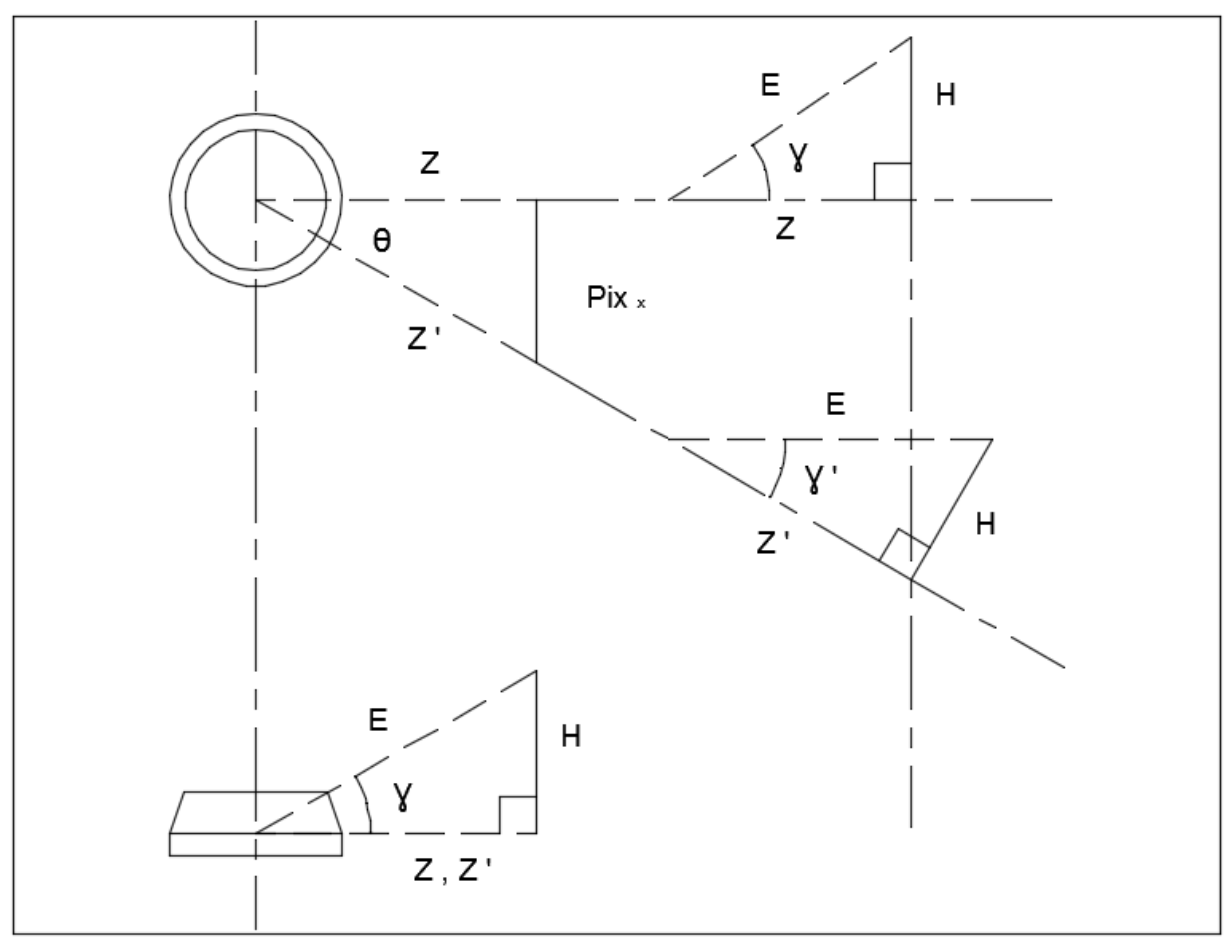

Figure 20: Single Camera Geometric Model

$$
\tan \gamma=\frac{H}{Z}
$$

Where $Z$ is the physical normal distance between the center of projection and the observed horizontal line alont the horizontal plane, $Z^{\prime}$ is the distance between the center of projection and any point on the observed curve along the horizontal plane, and $H$ is the physical elevation of the observed horizontal line. It is noted that the elevation reference is the elevation of the horizon of the lens projection. Based on the above equations, the relation between $\theta, \gamma$ and $\gamma^{\prime}$ is shown to be equal to

$$
\frac{\tan \gamma}{\tan \gamma^{\prime}}=\frac{Z^{\prime}}{Z}=\frac{1}{\cos \theta}
$$

As noted above, the rows of pixels in the unwrapped image correspond to the altitude angles of the given perspectives. This correspondence is assumed and validated to be linear for the used panoramic lens. In particular, the angular step size is 


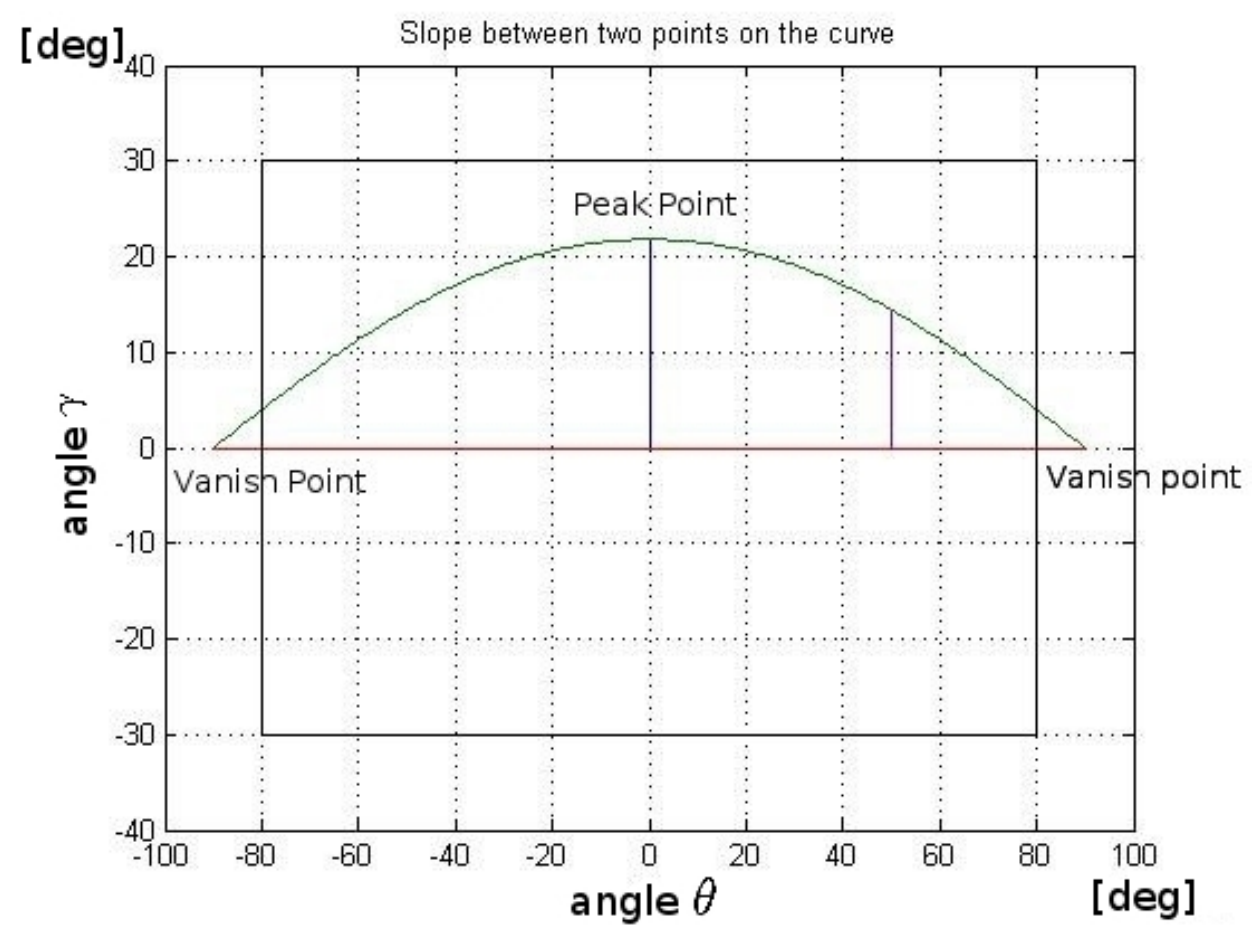

Figure 21: Vertical Angular Steps

constant along the vertical field of view. This vertical angular step size is evaluated per pixel by

$$
\text { angular step size per pixel }=\frac{\gamma}{b-F} \text {, }
$$

where $b$ is the horizon of the lens projection and $F$ is the vertical pixel's coordinate of the observed curve peak.

\subsection{Calibration}

The calibration process is essential for all vision systems because it identifies the cameras' intrinsic parameters. These parameters can be utilized to eliminate distortions in captured images. Intrinsic camera parameters depend on the geometry and design of the cameras and their corresponding lenses. In this section, the attention is focused on panoramic image calibration, i.e., identifying the intrinsic parameters of 


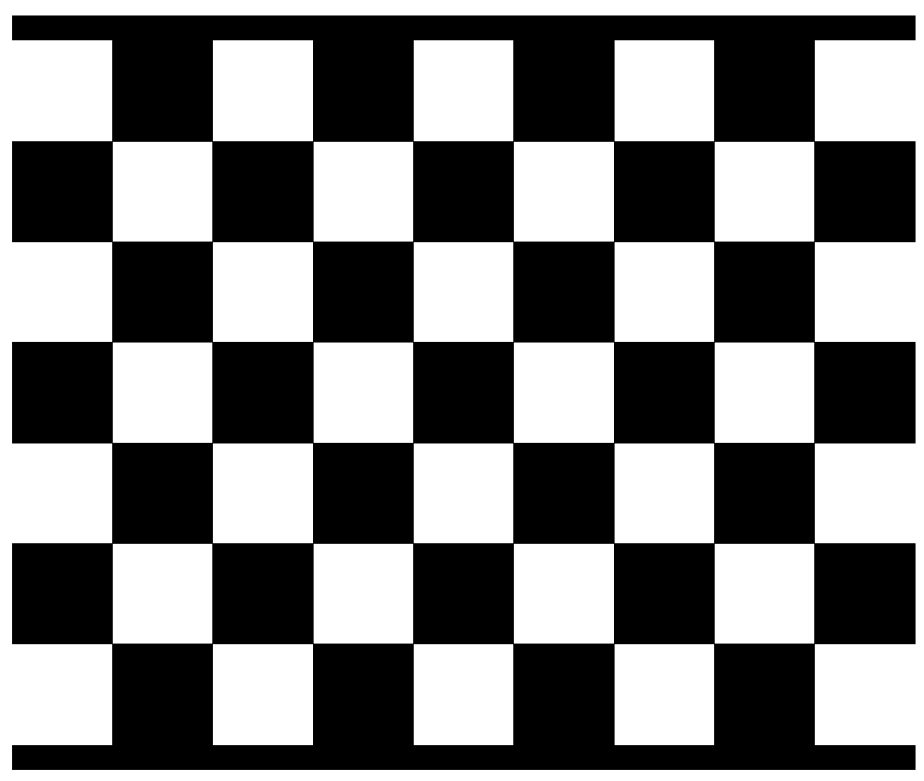

Figure 22: Calibration Board

a panoramic lens. The calibration of the conventional cameras is neglected and they are considered to be calibrated before the assembly of the panoramic lens.

The used folded lens has constant steps along the horizontal and vertical fields of view. Therefore, the intrinsic camera parameters of the panoramic lens are the horizon of the vertical visual field and the vertical angular step size. These parameters are independent of the vision system orientation.

To facilitate the calibration process, in this work, a new specially designed calibration board is proposed for calibrating unwrapped panoramic images. This calibration board is shown in Figure 22. and virtually simulated in figure 23. Unlike the conventional calibration boards, which have only corners, the proposed one has a combination of lines and corners. In particular, the unconventional top and bottom horizontal lines facilitate the identification of the intrinsic camera parameters through the usage of the geometric model proposed above.

Before performing the calibration, the board must be positioned in parallel with the vertical lens axis, whereas its horizontal lines must be physically perpendicular 


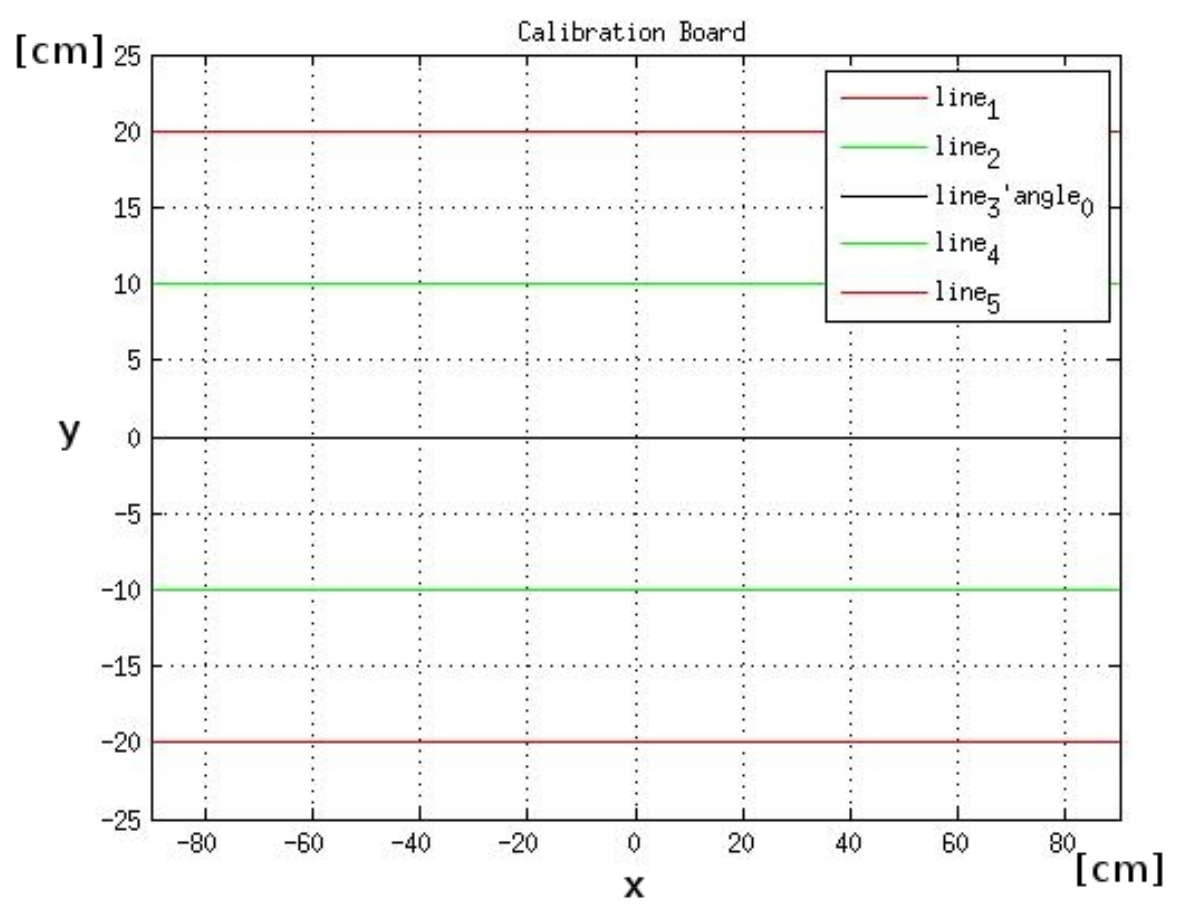

Figure 23: Horizontal Calibration Board Lines

to the vertical axis of the panoramic lens. To ensure that these conditions are met, every physical column of corners must share the same column of pixels on the unwrapped image frame. Assuming that these conditions are met, the horizontal lines of a calibration board are simulated in Figure 23 and their corresponding curves in the unwrapped image perspective in Figure 24.

Since the altitude angle is assumed to have constant steps in this work, it can be concluded that $\gamma$ is directly proportional to the vertical displacements $b-y$, where $b$ is the location of the horizon of the vertical visual field and $y$ is the vertical coordinate of a point observed at angle $\gamma$. Both $b$ and $y$ are presented in the image frame. This relation is illustrated in Equation (28), where $b-y$ and $b-y^{\prime}$ represent the vertical coordinates of two points along a curve at a physical altitude angle $\gamma$,

$$
\frac{b-y^{\prime}}{b-y} \times \gamma=\gamma^{\prime}
$$




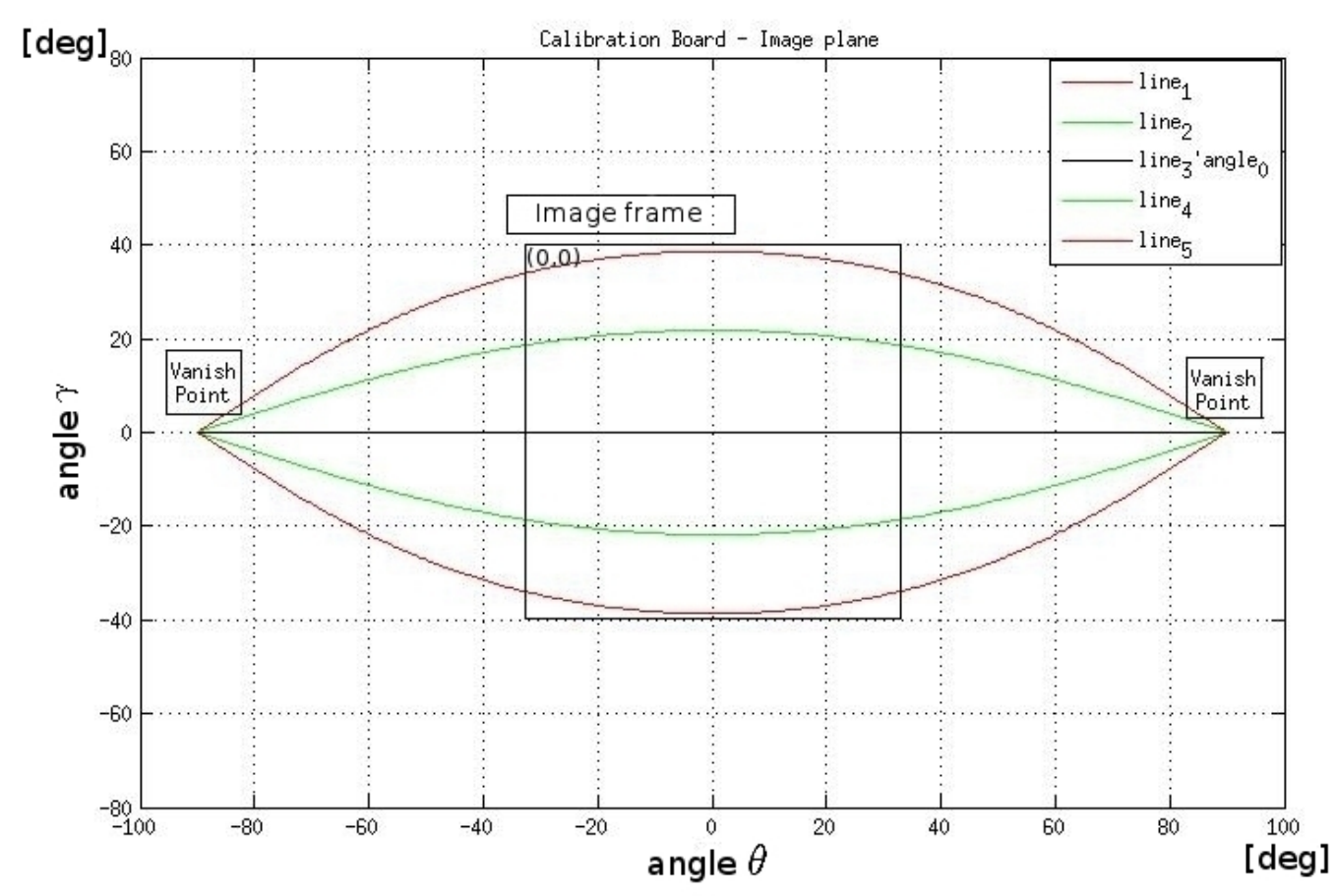

Figure 24: Horizontal Lines Perspectives

Subsequently, by substituting $\gamma^{\prime}$ in the geometric model of Equation (26), we obtain the relation

$$
\frac{\tan \gamma}{\tan \left(\frac{b-y^{\prime}}{b-y} \times \gamma\right)}=\frac{1}{\cos \theta} .
$$

Then this equation is rewritten to be

$$
\frac{\tan \gamma \times \cos \theta}{\tan \left(\frac{b-y^{\prime}}{b-y} \times \gamma\right)}-1=0
$$

In particular, this relation enables the evaluation of the altitude angular step size and the corresponding horizon of the vertical visual field $b$ that are essential for any further panoramic image processing. The proposed approach has proven to be very effective in calibrating panoramic view images and identifying their intrinsic parameters from their unwrapped panoramic view. 
In order to verify Equation (29), a virtual calibration board and its corresponding unwrapped image are simulated in this section. Using the provided equations, the angular perspective of the horizontal lines $\gamma$, horizon of the vertical visual field $b$, and the angular step size of the vertical field of view can be determined. Note that the main advantage of using simulations in this work is to allow the validation of the approach while neglecting the effects of other parameters, e.g., blurring effects, discrete pixel size, and feature detection errors. However, real life experiments are provided in the next section to validate the practicality of this equation.

In order to evaluate the performance of the proposed approach, it is initially tested on a simulated calibration board and its corresponding unwrapped view shown in Figure 24. Then Equation (29) was applied to simulate the observation of the horizontal lines of the calibration board as shown in Figure 24. In this simulation, the coordinates $y$ and $y^{\prime}$ were obtained from Figure 24 while Equation (28) is satisfied. In particular, the angles $\gamma$ and $\theta$ are directly proportional to the unwrapped image frame pixel coordinates. Therefore, the following is obtained:

$$
\begin{gathered}
y=40-38.66=1.34 \\
y^{\prime}(\theta=20)=40-36.93=3.07 \\
y^{\prime}(\theta=30)=40-34.72=5.28
\end{gathered}
$$

where the maximum observable vertical field of view along the unwrapped image frame is simulated at $80^{\circ}$. Moreover, the peak of the observed horizontal calibration board lines simulation is found at $38.66^{\circ}$.

Those output values were then substituted in Equation (30) and the resulting output was plotted in Figure 25. The variables $b$ and $\gamma$ were found to match the actual values shown in the Figure 24 . 


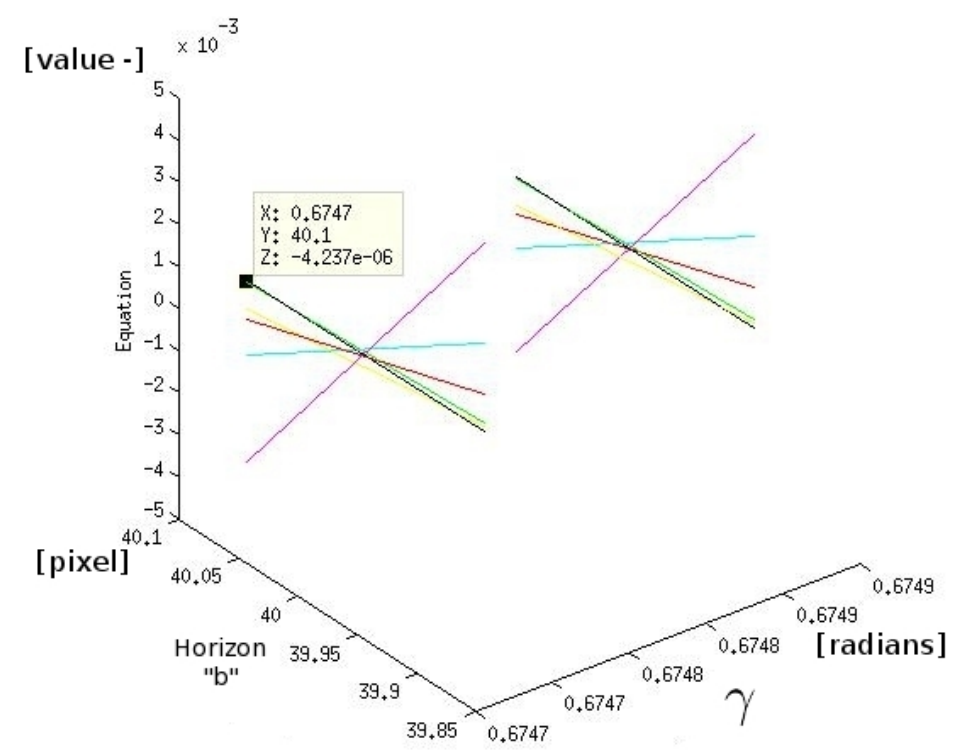

Figure 25: Image Parameters detection simulation

Each of the presented lines in Figure 25 corresponds to plane generated by a set of points on the curve of the unwrapped image view. In particular, every set of points is composed of the curve peak, i.e., $y$, and any point along the curve, i.e., $y^{\prime}$. Finally the combination of variables $b$ and $\gamma$ that satisfies the condition found in Equation (30) are determined by defining the planes points of intersection.

\subsubsection{Experiments}

In the provided experiment, a raspberry-pi camera module is used. This module has a 5 mega pixel sensor, i.e., $2592 \times 1944$ pixels, with a pixel size of $0.0014 \mathrm{~mm} \times 0.0014 \mathrm{~mm}$. The lens has a diameter of $1.25 \mathrm{~mm}$ that is found to provide a $53^{\circ}$ horizontal field of view and a $40^{\circ}$ vertical field of view. Moreover, this camera generates Joint Photographic Experts Group (JPEG) image extensions.

A folded catadioptric lens with undefined parameters, shown in Figure 27, is 


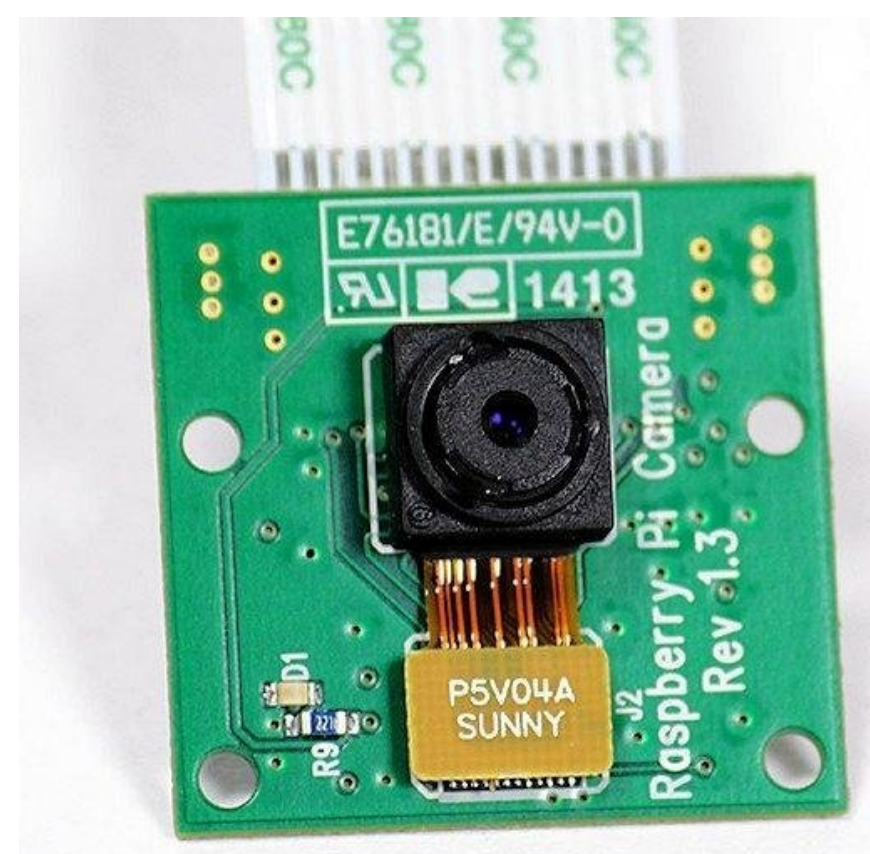

Figure 26: Raspberry-Pi Camera Module

used with the raspberry-pi camera module. However, the vertical angular steps of the unwrapped panoramic image are tested and verified to be constant along a $60^{\circ}$ vertical field of view. Moreover, the provided setup allows us to prove the feasibility of the calibration approach.

The primary and secondary perspectives of the calibration board corners were found to be as follows:

Primary image corners: 


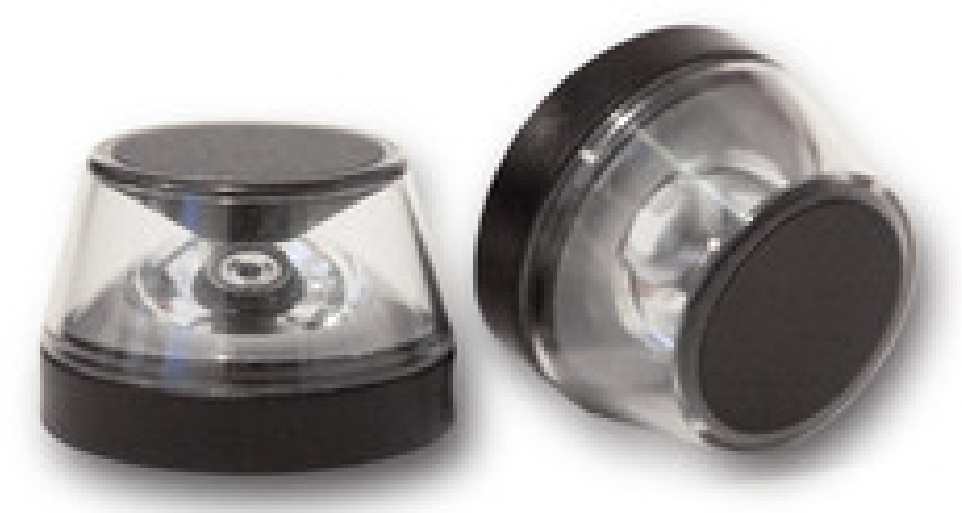

Figure 27: Kogeto Dot Panoramic Lens

\begin{tabular}{lcccccccc} 
row $/$ col & 1 & 2 & 3 & 4 & 5 & 6 & 7 & 8 \\
\hline 1 & 406,63 & 443,75 & 484,51 & $528,45.5$ & $577,40.5$ & 629,37 & 682,36 & 734,37 \\
2 & 406,87 & 443,83 & 484,77 & 528,73 & 577,70 & 629,66 & 682,65 & 734,66 \\
3 & 406,113 & 443,110 & $484,105.5$ & $529,101.5$ & $578,98.5$ & 629,97 & 682,95 & 735,97 \\
4 & 407,140 & 444,137 & $485.5,134$ & $529,131.5$ & 578,130 & 629,129 & $682.5,129$ & 735,128 \\
5 & 407,164 & 444,163 & $485.5,162$ & $529.5,161$ & 578,161 & 630,160 & $683,160.5$ & 736,160 \\
6 & $407.5,189$ & 444,190 & $485.5,190$ & $529.5,190$ & 579,191 & $630,191.5$ & $683.5,191.5$ & 736,191 \\
\hline
\end{tabular}

Secondary image corners: 


\begin{tabular}{lcccccccc} 
row/col & 1 & 2 & 3 & 4 & 5 & 6 & 7 & 8 \\
\hline 1 & 572,42 & 624,39 & 678,40 & 730,38 & 782,42 & 831,45 & 875,50 & 916,56 \\
2 & 573,71 & 624,69 & 678,67 & 731,68 & 782,69 & 832,74 & 877,76 & 917,83 \\
3 & 573,101 & 625,99 & 680,100 & 731,99 & 784,101 & 832,102 & 877,105 & 917,109 \\
4 & 574,133 & 625,132 & 680,131 & 733,132 & 784,131 & 833,134 & 878,134 & 918,138 \\
5 & 575,165 & 627,164 & 679,165 & 732,164 & 785,165 & 834,163 & 879,166 & 918,165 \\
6 & 576,194 & 628,195 & 681,195 & 734,196 & 786,194 & 834,194 & 880,192 & 919,194 \\
\hline
\end{tabular}

The calibration board is checked to be parallel to the lens vertical axis. Therefore, every physical column of corners have to share the same column of pixels. However, a small variation of up to 4 pixels found in the two perspectives is neglected. Moreover, the peak point of one of the two curves, i.e., the upper and lower curves, is chosen along with any two points on the curve. These two sets of points are used to define two planes from Equation (29). The intersection of these planes allows us to define the vertical field of view at the peak point as well as the center of the vertical visual field. However, the accuracy of the chosen calibration points coordinates is affected by the size of pixels, contrast, and the unwrapping accuracy. Therefore, the furthest detectable points along the curve are chosen to increase the curve slope and reduce the percentage of the calibration errors. Then, the angle $\gamma$ and the horizon of the vertical visual field $b$ of the two images are defined with two sets of points in each image as shown in Equation (29) while the dimensions of the unwrapped images are $2701 \times 281$.

For the first image, the peak curve point has the coordinates $(675,4)$ and the two other chosen curve points have coordinates $(481,21)$ and $(440,29)$. These coordinates are substituted in Equation (29) as follows:

$$
\frac{\tan \gamma}{\tan \left(\frac{b-21}{b-4} \times \gamma\right)}=\frac{1}{\cos \left(\frac{675-481}{2701} \times 2 \times p i\right)}
$$




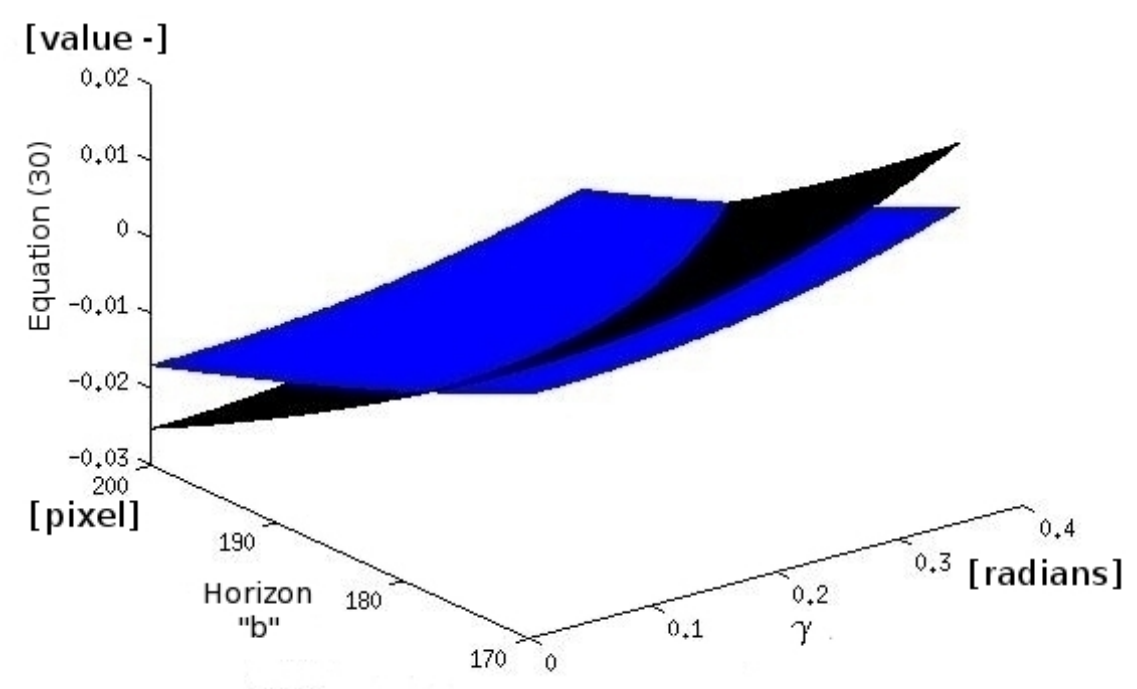

Figure 28: First Camera Intrinsic Parameters

$$
\frac{\tan \gamma}{\tan \left(\frac{b-29}{b-4} \times \gamma\right)}=\frac{1}{\cos \left(\frac{675-440}{2701} \times 2 \times p i\right)}
$$

From previous equation substitutions, Figure 28 is generated and the angle $\gamma$ is defined to be in the range 0.2 to 0.4 and coordinate $b$ to be in the range between 180 to 190 .

For the second image, the Equation (29) is substituted with the curve peak point $(675,8)$ and the curve points $(815,14)$ and $(876,23)$ as follows:

$$
\begin{aligned}
& \frac{\tan \gamma}{\tan \left(\frac{b-23}{b-4} \times \gamma\right)}=\frac{1}{\cos \left(\frac{675-876}{2701} \times 2 \times p i\right)} \\
& \frac{\tan \gamma}{\tan \left(\frac{b-14}{b-4} \times \gamma\right)}=\frac{1}{\cos \left(\frac{675-815}{2701} \times 2 \times p i\right)}
\end{aligned}
$$

The second set of equations generate Figure 29. These equations define the angle $\gamma$ to be in the range 0.3 to 0.8 , and the coordinate $b$ to be in the range between 175 


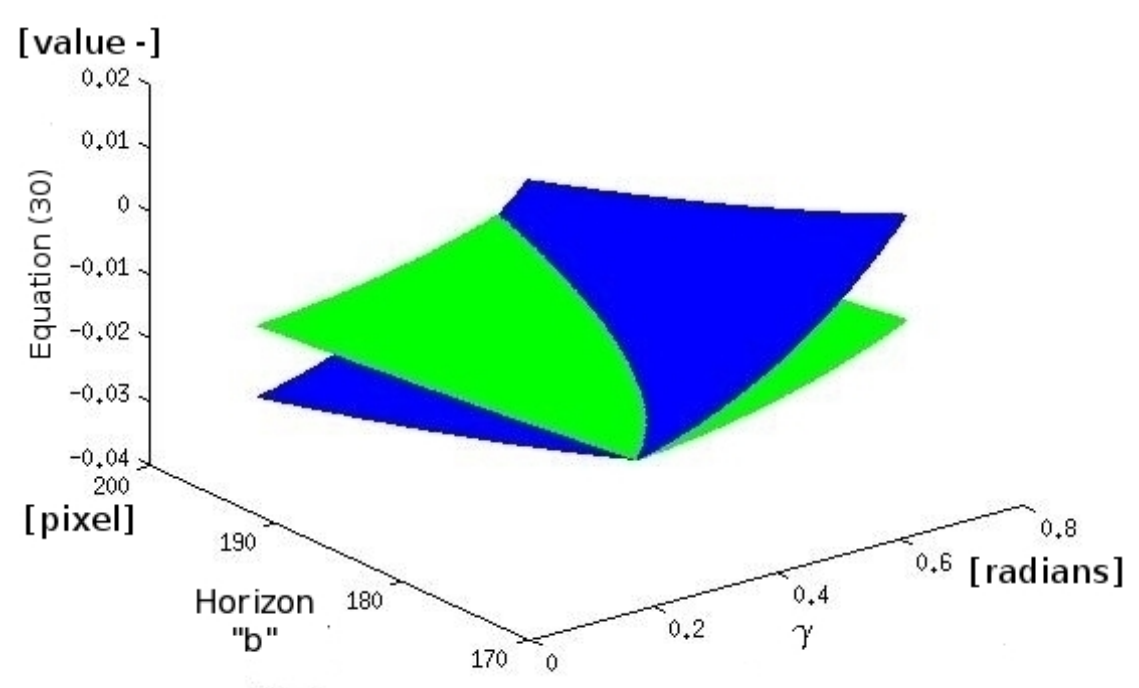

Figure 29: Second Camera Intrinsic Parameters

to 200 .

From previous simulations, it is concluded that the curve points identification process is essential for the camera calibration process. In particular, the quality and size of the image are essential to reduce the errors and increase the accuracy of the coordinates identification process. However, the points far from the curve peak are the best points to choose because these points decrease the percentage of error in the calibration process by increasing the slope of the curve.

Moreover, the flowchart shown in Figure 30 is provided to further illustrate the calibration process. 


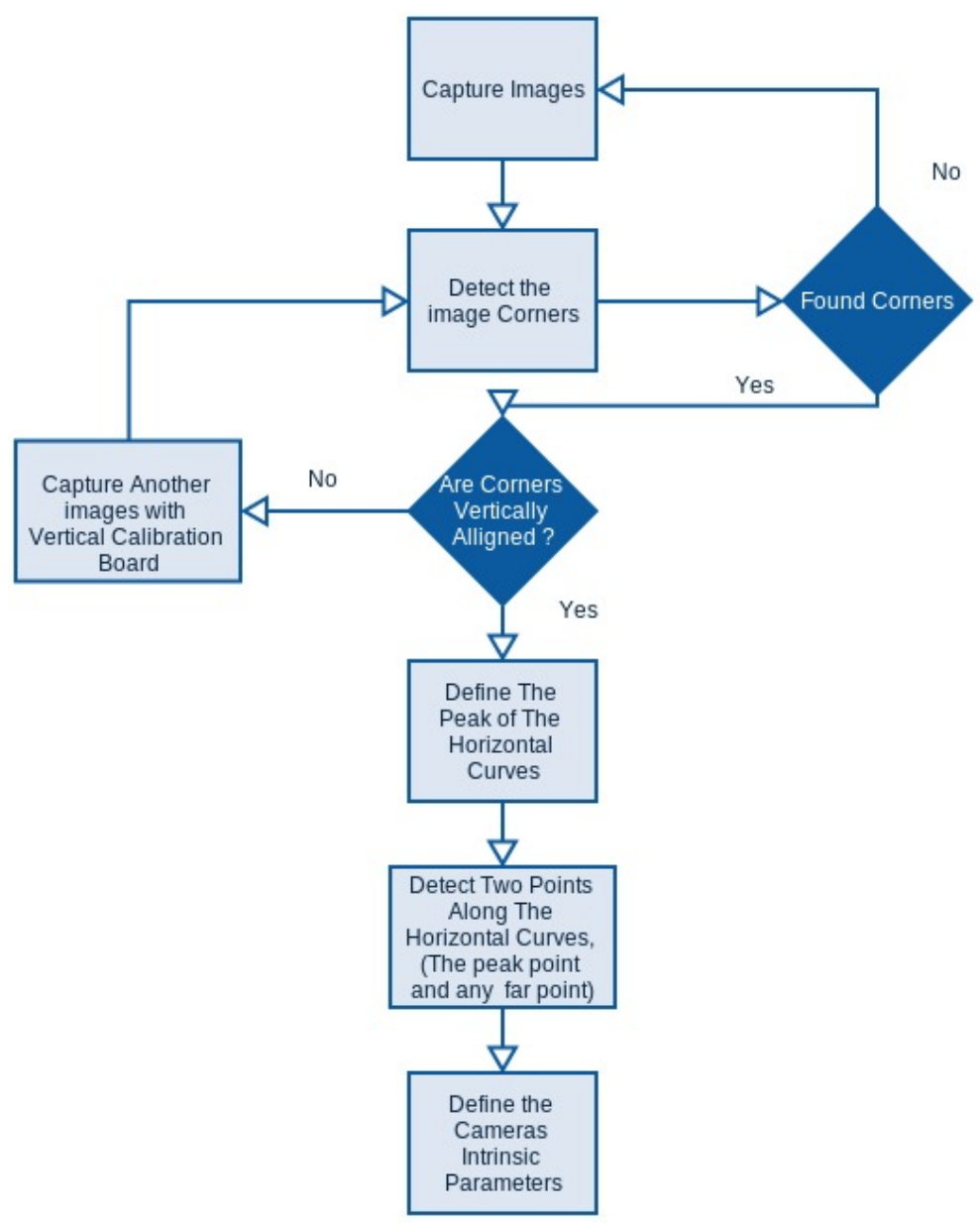

Figure 30: Calibration Flowchart 


\section{Chapter 5}

\section{Stereo Panoramic Imaging}

\subsection{Introduction}

Stereo vision systems allows the robots to acquire 3-D virtual maps of their surroundings. The provided 3D map allows the robot to sense its workspace objects and to navigate safely within its surrounding environment. Conventional stereo vision systems capture a 3D map of their surroundings in a specific perspective. In some circumstances, mobile robots can face a featureless perspective. In such a case, the perception system will face the problem of losing the observed features correspondence. Unlike the conventional systems, panoramic stereo vision systems provide the robot with a panoramic 3D map of its environment. The panoramic 3D map allows the robot to continuously capture the environment information from different perspectives during its operation and thus reducing the probability of facing a featureless scene. Stereo panoramic imaging systems have different setups with different properties. In particular, such systems have different formats, i.e., either a side-to-side or a co-axial. These formats are discussed next.

\subsubsection{Coaxial Format}

The coaxial format of stereo panoramic vision systems capture two panoramic 


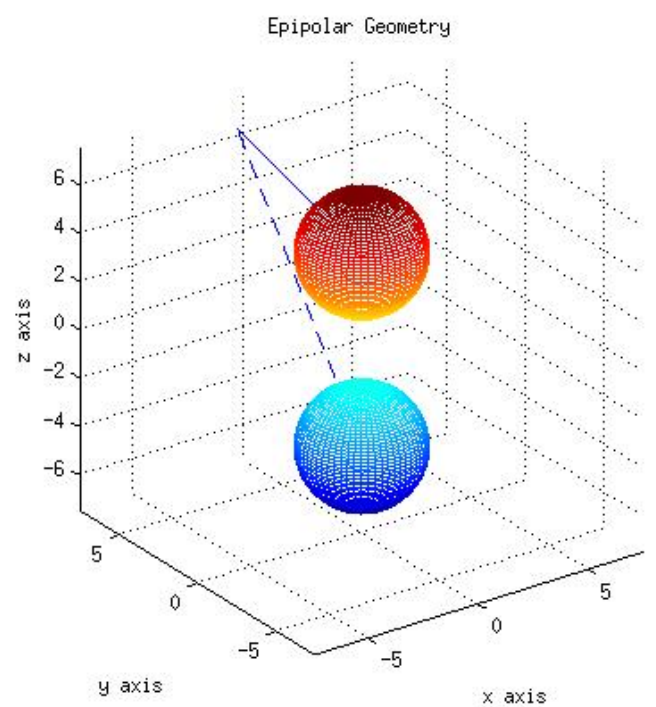

Figure 31: Epipolar Geometry Model - Coaxial Format

images from different altitudes. Such systems have a common panoramic lens axis. Moreover, coaxial formats have an advantageous epipolar constraints, i.e., the observed features are rectified vertically, as shown in Figure 31. Using this format, the 3D environment reconstruction can be provided by vertically triangulating the correspondent features disparity in the provided panoramic perspectives.

\subsubsection{Side-To-Side Format}

Side-to-side stereo panoramic vision systems capture panoramic images from different orientations at a common altitude. Such systems can be used for low profile robot systems. One example of such application can be the indoor service robots, e.g., vacuum cleaning robots.

\section{$5.2 \quad$ Epipolar Geometry}

Epipolar geometry is the geometric relation between two independent perspectives 


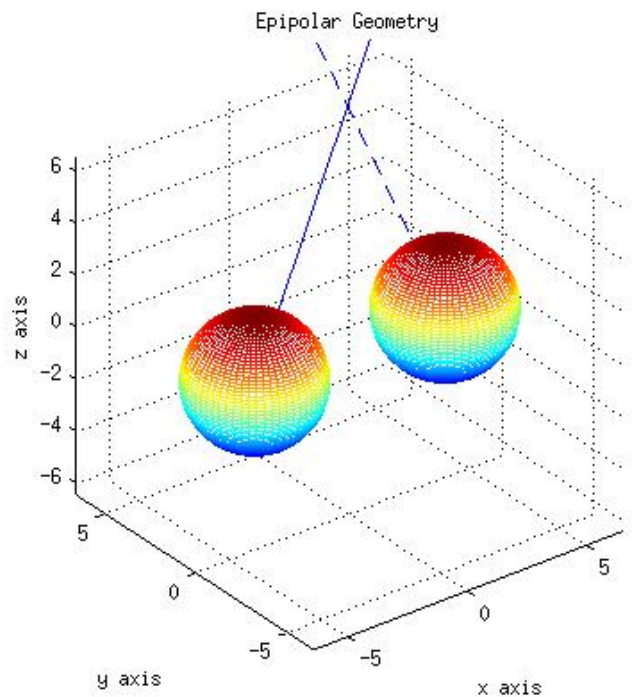

Figure 32: Epipolar Geometry Model - Side-To-Side Format

of a 3D scene. In this work, the proposed geometric model is based on two unwrapped image frames captured from a side-to-side stereo panoramic vision system. In the considered system, the azimuth angle of the primary and secondary lenses are denoted by $\theta_{1}$ and $\theta_{2}$, respectively. Similarly, the altitude angles of the primary and secondary lenses are denoted by $\gamma_{1}$ and $\gamma_{2}$. These four angles will be projected from the two lenses towards a feature in the 3D scene.

To search for the desired feature, the proposed approach sets a projection of $\theta_{1}$ and $\gamma_{1}$ and then searches for all possible combinations of $\theta_{2}$ and $\gamma_{2}$ that satisfy the geometric model shown in Figure 33. The search plan of $\theta_{2}$ and $\gamma_{2}$, i.e., the epipolar curve, is derived from the geometric model in Figure 33 as follows:

From the rule of sines

$$
\frac{L_{1}}{\sin \theta_{2}}=\frac{L_{2}}{\sin \theta_{1}}=\frac{d}{\sin \left(180-\theta_{1}-\theta_{2}\right)} .
$$


Using this equation, we arrive at

$$
\begin{gathered}
L_{1}=\frac{d \times \sin \theta_{2}}{\sin \left(180-\theta_{1}-\theta_{2}\right)}, \text { and } \\
L_{2}=\frac{d \times \sin \theta_{1}}{\sin \left(180-\theta_{1}-\theta_{2}\right)} .
\end{gathered}
$$

Using the 3D model shown in Figure 34, we arrive at

$$
\begin{gathered}
\tan \gamma_{1}=\frac{H}{L_{1}}, \\
\tan \gamma_{2}=\frac{H}{L_{2}} \text {, and } \\
H=L_{1} \tan \gamma_{1}=L_{2} \tan \gamma_{2} .
\end{gathered}
$$

Subsequently,

$$
\begin{gathered}
\gamma_{2}=\tan ^{-1}\left(\frac{H}{L_{2}}\right)=\tan ^{-1}\left(\frac{\left(\frac{d \times \sin \theta_{2}}{\sin \left(180-\theta_{1}-\theta_{2}\right)}\right) \times \tan \gamma_{1}}{\left(\frac{d \times \sin \theta_{1}}{\sin \left(180-\theta_{1}-\theta_{2}\right)}\right)}\right), \text { and } \\
\gamma_{2}=\tan ^{-1} \frac{\sin \theta_{2} \tan \gamma_{1}}{\sin \theta_{1}}
\end{gathered}
$$

From the above derivation, the epipolar curve was found to be a sinusoidal curve around the horizon of the unwrapped perspective, i.e., $\gamma=0$.

Using the four angles, $\gamma_{1}, \theta_{1}, \gamma_{2}$ and $\theta_{2}$, the depth of a 3D scene feature can be evaluated. In particular, a method for calculating the physical depth of a given feature in the $3 \mathrm{D}$ scene is developed in this work by using triangulation. Using this method, both the horizontal depth Depth $h_{x y}$ and vertical depth $D e p t h_{x z}$ of a given feature can be evaluated. The equations of these physical depths can be derived based on the model found in Figure 33 as follows: 


$$
\begin{gathered}
\tan \gamma_{1}=\frac{H}{L_{1}} \\
\tan \gamma_{2}=\frac{H}{L_{2}} \\
H=L_{1} \tan \gamma_{1}=L_{2} \tan \gamma_{2} \\
D e p t h_{x y}=d_{1} \tan \theta_{1}=d_{2} \tan \theta_{2}
\end{gathered}
$$

Where $d_{1}$ and $d_{2}$ are shown in Figure 33 .

To evaluate the Dept $h_{x y}$, four scenarios can occur. First, if $\left|\theta_{1}\right|$ is greater than $90^{\circ}$, then

$$
\begin{gathered}
d=L_{1} \cos \theta_{1}+L_{2} \cos \theta_{2}, \text { and } \\
\text { Depth }_{x y}=\frac{d \tan \theta_{2} \tan \theta_{1}}{\tan \theta_{1}-\tan \theta_{2}} .
\end{gathered}
$$

Where $d$ is the physical distance between the two cameras, i.e., stereo camera's baseline. Second, if $\left|\theta_{1}\right|$ is less than $90^{\circ}$, then

$$
\begin{gathered}
d=L_{2} \cos \theta_{2}-L_{1} \cos \theta_{1}, \text { and } \\
\text { Depth }_{x y}=\frac{d \tan \theta_{2} \tan \theta_{1}}{\tan \theta_{1}+\tan \theta_{2}} .
\end{gathered}
$$

Third, if $\left|\theta_{1}\right|=90^{\circ}$, then

$$
\operatorname{Depth}_{x y}=d \tan \theta_{2}
$$

Fourth, if $\left|\theta_{2}\right|=90^{\circ}$, then

$$
\operatorname{Depth}_{x y}=d \tan \theta_{1}
$$

irrespective of the value of $\theta_{1}$.

Similarly, when evaluating Depth $h_{x z}$, three scenarios can occur. First, if $\left|\theta_{1}\right|=90^{\circ}$, 


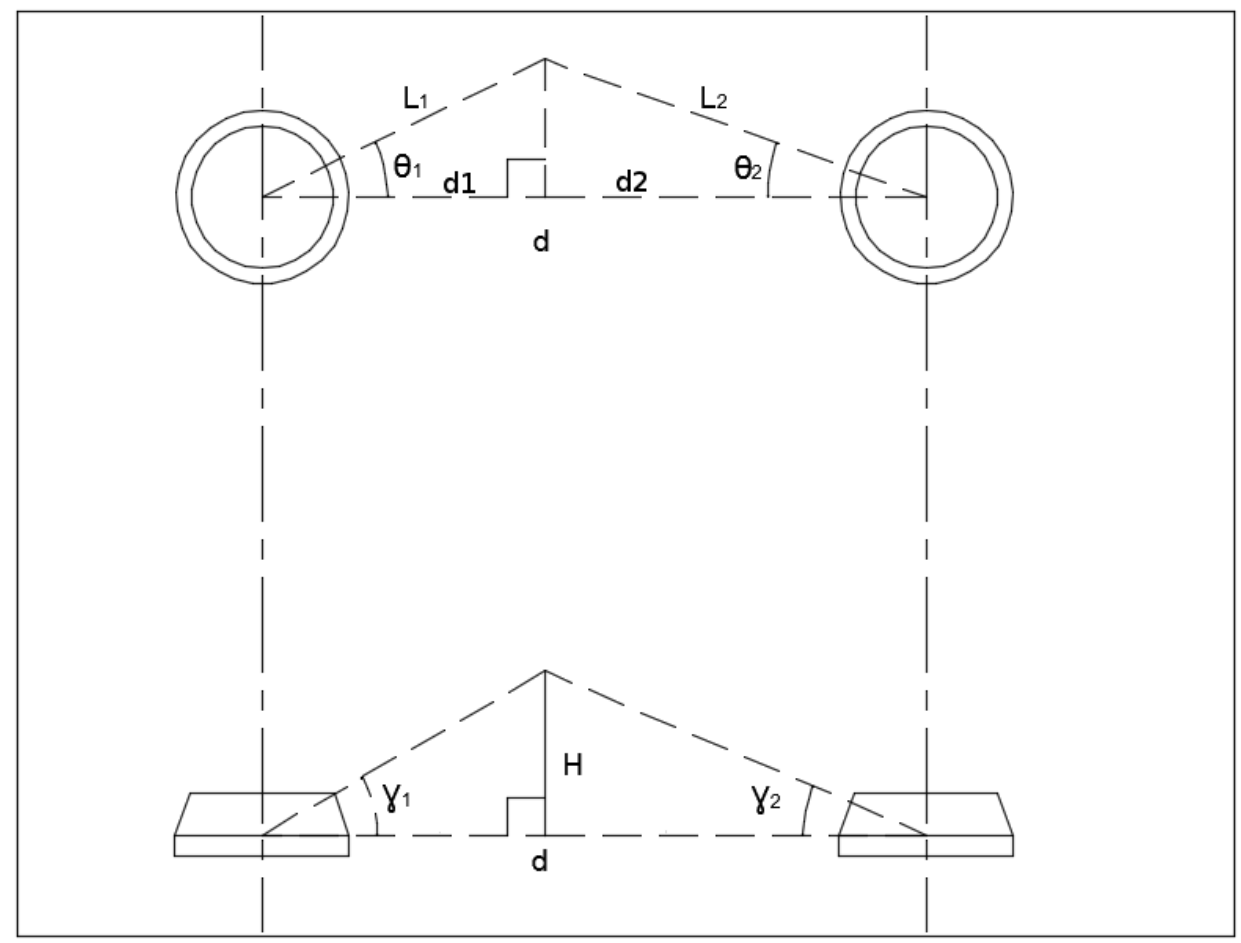

Figure 33: Panoramic Epipolar Geometry Model

then

$$
\operatorname{Depth}_{x z}=\tan \gamma_{2} \frac{d}{\cos \theta_{2}} .
$$

Second, if $\left|\theta_{2}\right|=90^{\circ}$, then

$$
\operatorname{Depth}_{x z}=\tan \gamma_{1} \frac{d}{\cos \theta_{1}} .
$$

Otherwise,

$$
\operatorname{Depth}_{x z}=\tan \gamma L_{1},
$$

where in this scenario, $L_{1}=\frac{\operatorname{Depth}_{x y}}{\sin \theta_{1}}$. 


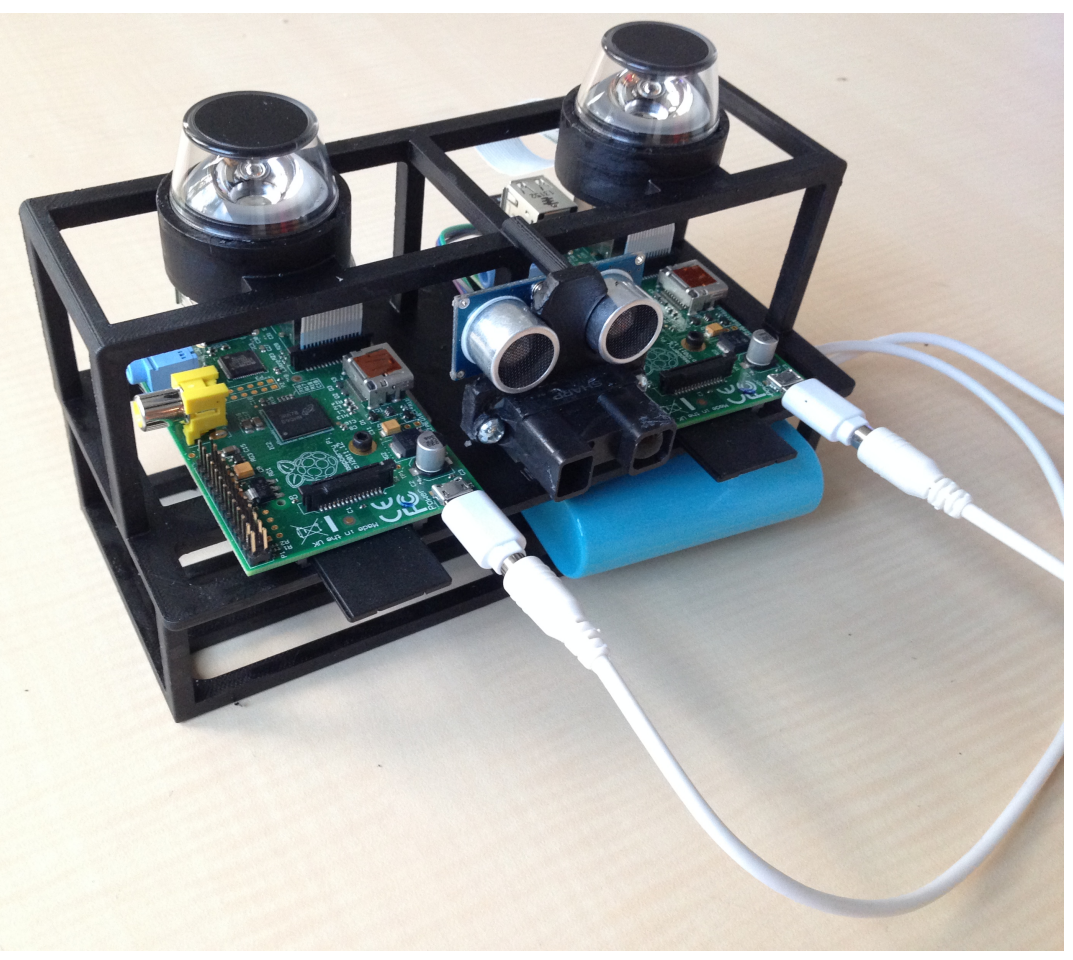

Figure 34: Camera Setup

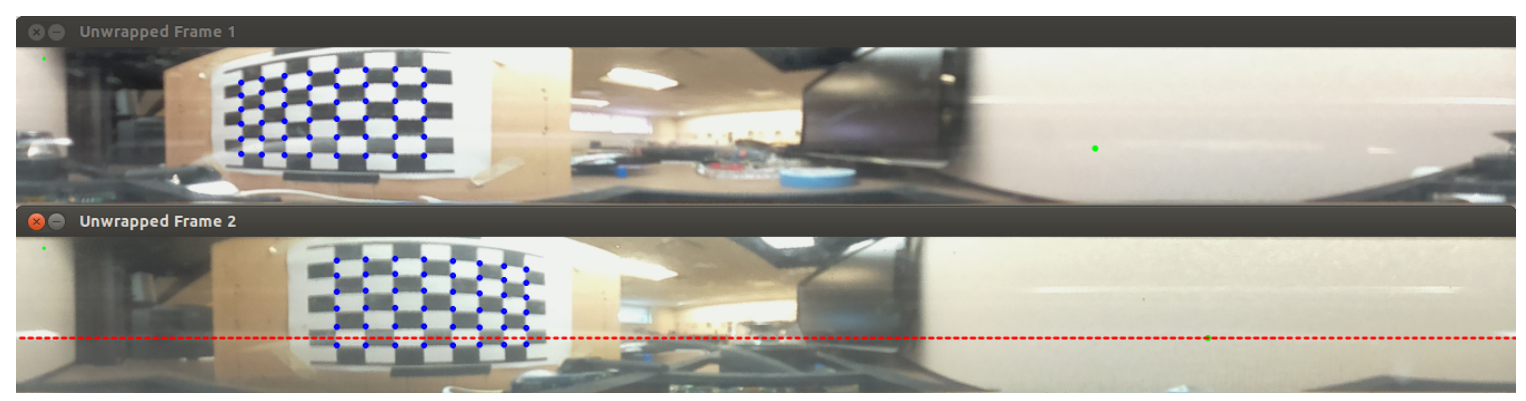

Figure 35: Corners Mask

\subsection{Stereo Transformation}

Stereo vision transformation is the process of finding the relation between different views on a stereo vision system by defining its extrinsic parameters. Extrinsic parameters are dependent on the relative orientations of the stereo setup. To find the transformation between the two images, two image perspectives observing a predefined calibration pattern are used. In particular, a reference image is chosen and 
the transformation matrix of the second images with respect to the reference image is defined. This transformation matrix is composed of translational and rotational components. The translation matrix, $T$, represents the axial motion of the lens center axis, whereas the rotation matrix, $R$, represents the rotation about the three axis. Both, the translational and rotational transformations are briefly discussed next.

\section{Translation}

Since any panoramic image provides a $360^{\circ}$ perspective of its surrounding environment, it can not have a translation in the $x-y$ horizontal frame. However, it can still have a translation along the $z$-axis, i.e., the center axis of the panoramic image. Along this axis, a translation matrix will represent a shift $t$ between the altitudes of the two panoramic lenses as shown in Figure 36. Therefore, the secondary image will have a translation matrix $T$. It is noted that, this translation is always associated with the secondary image since the primary image is taken as a reference. One example of a translation matrix can be given by

$$
T=\left[\begin{array}{l}
x=0 \\
y=0 \\
z=t
\end{array}\right] .
$$

\section{Rotation}

Rotations in a 3D world can have up to three degrees of freedom. Such rotations are usually denoted in robotics as roll, pitch, and yaw. In this system, the angle $\theta$ corresponds to the yaw rotation about $z$ axis, whereas the angle $\gamma$ corresponds to 


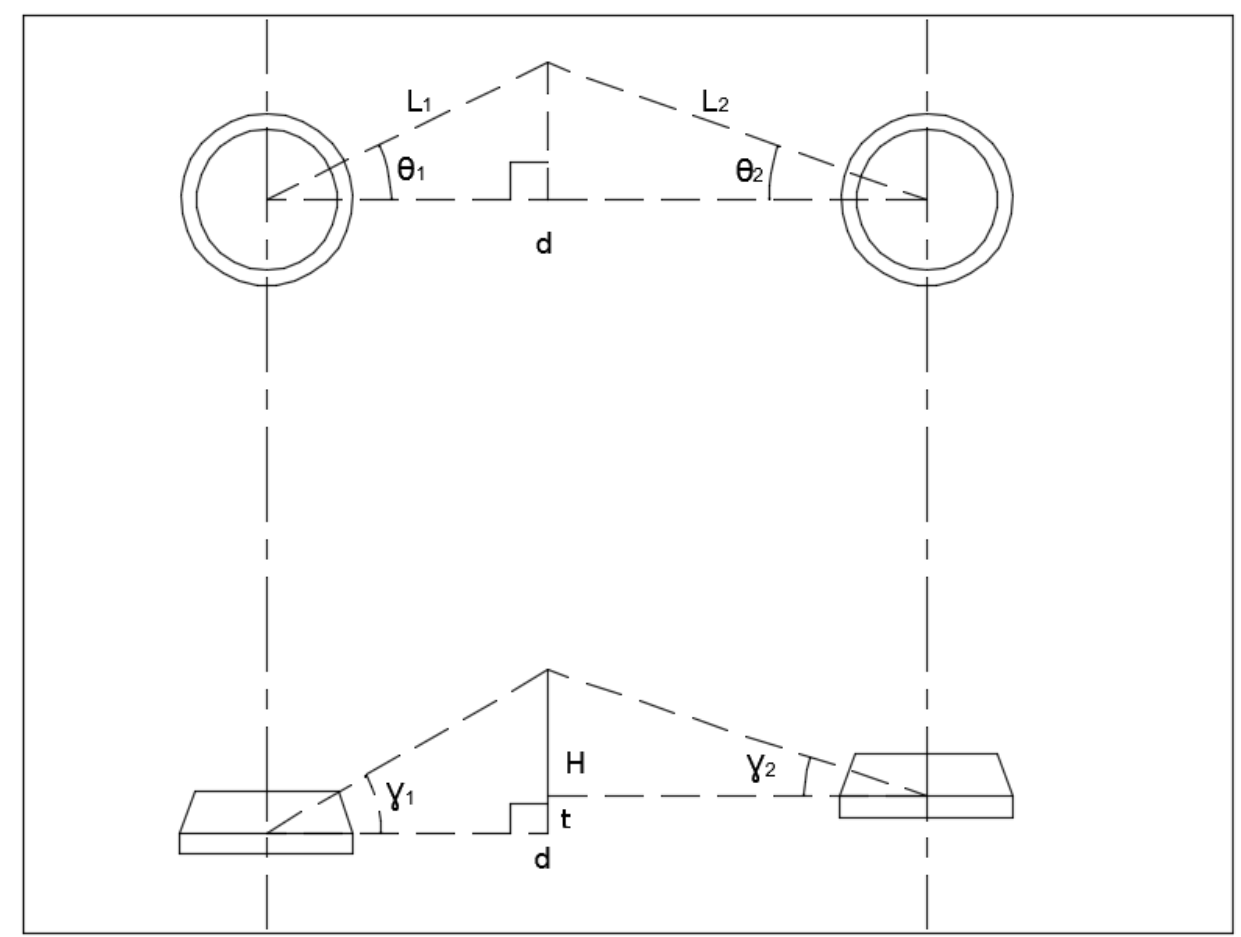

Figure 36: Detailed Geometric Model - Translation

the pitch angle. An example of a stereo vision system with rotation is provided in Figure 37.

Since the proposed system is free to rotate in the 3D world, one representation of its total rotation, denoted by $R_{\text {total }}$, can be given by

$$
R_{\text {total }}=R_{z}\left(\theta^{\prime}\right) \times R_{x}(\gamma) \times R_{z}(\theta)
$$

Where $\theta^{\prime}$ is the second rotation about the $z$ axis, and $R_{x}$ and $R_{z}$ represent the rotation about the $x$ and $z$ axis, respectively. These rotation matrices are expressed in terms of Euler angles as follows: 


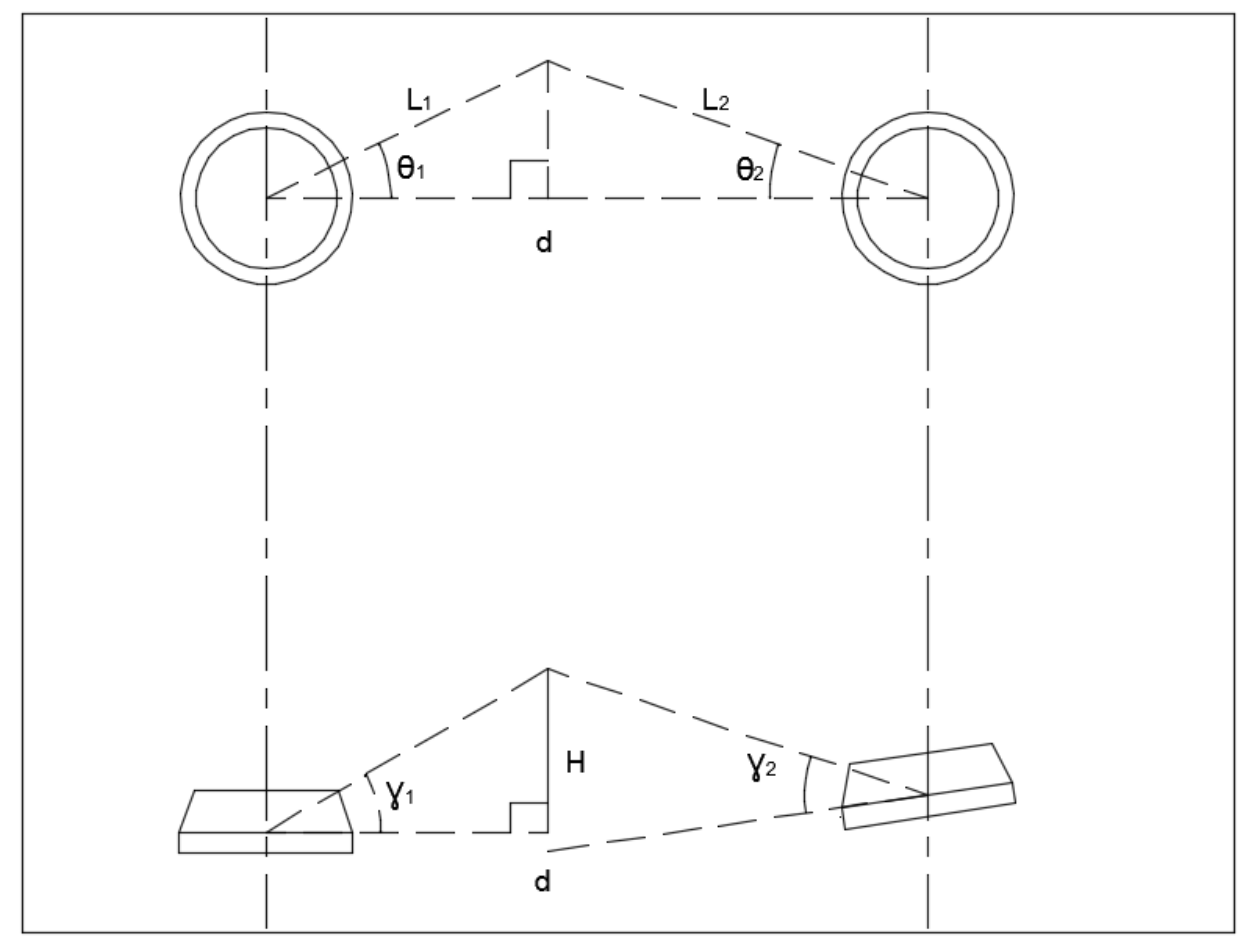

Figure 37: Detailed Geometric Model - Rotation

$$
\begin{aligned}
R_{x}= & {\left[\begin{array}{ccc}
1 & 0 & 0 \\
0 & \operatorname{Cos}(\gamma) & -\operatorname{Sin}(\gamma) \\
0 & \operatorname{Sin}(\gamma) & \operatorname{Cos}(\gamma)
\end{array}\right], \text { and } } \\
R_{z} & =\left[\begin{array}{ccc}
\operatorname{Cos}(\theta) & -\operatorname{Sin}(\theta) & 0 \\
\operatorname{Sin}(\theta) & \operatorname{Cos}(\theta) & 0 \\
0 & 0 & 1
\end{array}\right] .
\end{aligned}
$$

It is noted that, the above considered rotational transformation can be decomposed into three sequential rotations. The first rotation is about the $z$ axis with angle 
$\theta$, whereas the second rotation is about the $x$ axis with angle $\gamma$, and finally the third rotation is about the $z$ axis with angle $\theta^{\prime}$ in a direction opposite to that of the first rotation.

After applying the matrix multiplication in Equation (53), $R_{\text {total }}$ can be given by

$$
R_{z^{\prime} x z}=\left[\begin{array}{ccc}
C_{\theta^{\prime}} C_{\theta}-C_{\gamma} S_{\theta^{\prime}} S_{\theta} & -C_{\theta^{\prime}} S_{\theta}-C_{\gamma} C_{\theta} S_{\theta^{\prime}} & S_{\theta^{\prime}} S_{\gamma} \\
C_{\theta} S_{\theta^{\prime}}+C_{\theta^{\prime}} C_{\gamma} C_{\theta} & C_{\theta^{\prime}} C_{\beta} C_{\theta}-S_{\theta^{\prime}} S_{\theta} & -C_{\theta^{\prime}} S_{\gamma} \\
S_{\gamma} S_{\theta} & C_{\theta} S_{\gamma} & C_{\gamma}
\end{array}\right],
$$

where $C_{i}$ is $\cos (i)$ and $S_{i}$ is $\sin (i)$.

Due to design and assembly imperfections in practical panoramic stereo setups, a panoramic camera might undergo rotations and translations with respect to the other camera. The effects of these imperfections can be countered by applying a rotational and translational transformations as

$$
X_{\text {alligned }}=\left[R_{\text {total }}\right]\left[X_{\text {imp }}\right]+[T]
$$

Where $X_{\text {alligned }}$ and $X_{i m p}$ are the ideal and the misaligned orientations of the secondary panoramic camera with respect to the reference camera, respectively. In order to obtain these rotational and translational transformations, the observed calibration board correspondent corners are used with their estimated positions in the secondary image. In particular, at least four corresponding corners are required since four variables need to be defined, i.e., $\theta, \theta^{\prime}, \gamma$, and $t$.

Fortunately, the used 3D printed setup had a negligible misalignment error in the locations of the observed corners. This was proven by comparing the location where the corners were observed with the location where they are supposed to be found through applying an ideal mask of corners, i.e., a mask of corners at their 
estimated ideal positions. The proposed 3D printed setup has proven to be in the ideal orientation as shown in Figure 35. Subsequently, this eliminated the need for defining the rotational and translational transformation matrices in this setup.

\subsubsection{Rectification}

The images rectification process helps in reducing the complexity of identifying the feature disparity in a stereo setup. This is done through reducing the $2 \mathrm{D}$ correspondence problem to a $1 \mathrm{D}$ problem. For example, it rectifies the image frames captured by conventional stereo vision systems to be row aligned. Unfortunately, the usage of rectification in the proposed side-to-side panoramic vision system can not be computationally justified. This is because, in this study, the epipolar geometry shows that every column of pixels would have a special set of correspondent sinusoidal epipolar curves on the second image. For example, in the proposed system, the unwrapped images are of size 2701 by 289 pixels. Thus, in order to apply the stereo images rectification process, 2701 rectified images would be needed.

\subsection{Simulations and Experiments}

In the following simulations, numerical evaluations of the epipolar correspondence and depth efficiency are provided for different feature depths. In addition, experimental discussion on the effect of the 3D printed material transparency and the used camera's resolution on both the lens calibration process and the epipolar geometry correspondence are provided.

\subsubsection{D Print Material}

In this work, a 3D printed camera setup with a transparent ivory plastic material 


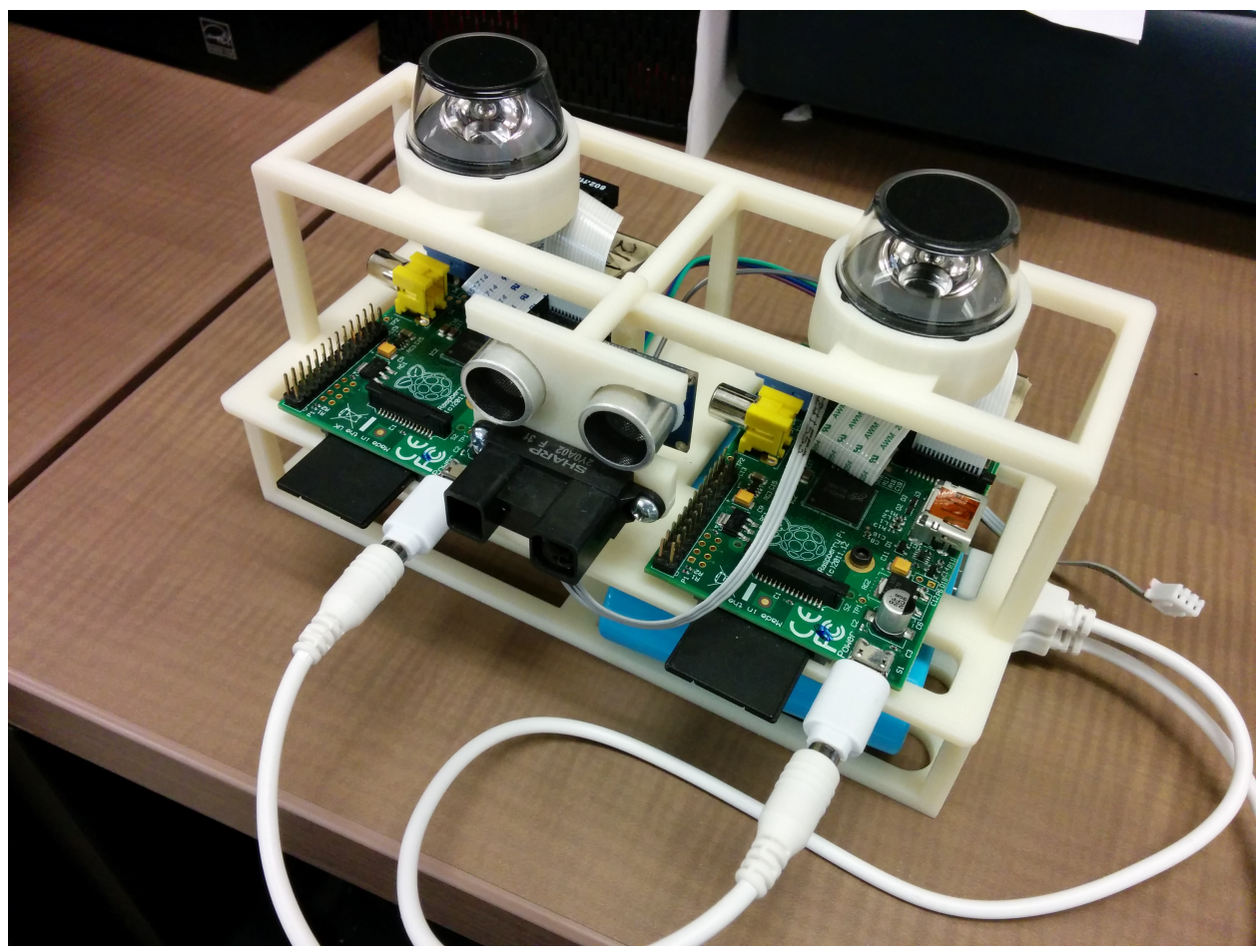

Figure 38: Stereo Camera Setup Before Being Applying Dark Paint

is used as shown in Figure 38. This transparency enabled light penetration through the plastic material which resulted in light related image distortions. To mitigate these distortions, a black paint was applied to the model to reduce or eliminate the light effects.

To show the effect of the proposed solution, a before and after captured images in Figure 39 are presented. From this figure, it can be easily noticed that the dark paint has been successful in significantly reducing the light effects. Subsequently, the captured image quality was improved.

\subsubsection{Resolution Effects}

In the proposed system, it was experimentally observed that the variation of the pixels coordinates in the provided image can reach up to 6 pixels. Since the provided images have a width of 2701 pixels and a height of 289 pixels, the error per pixel in 


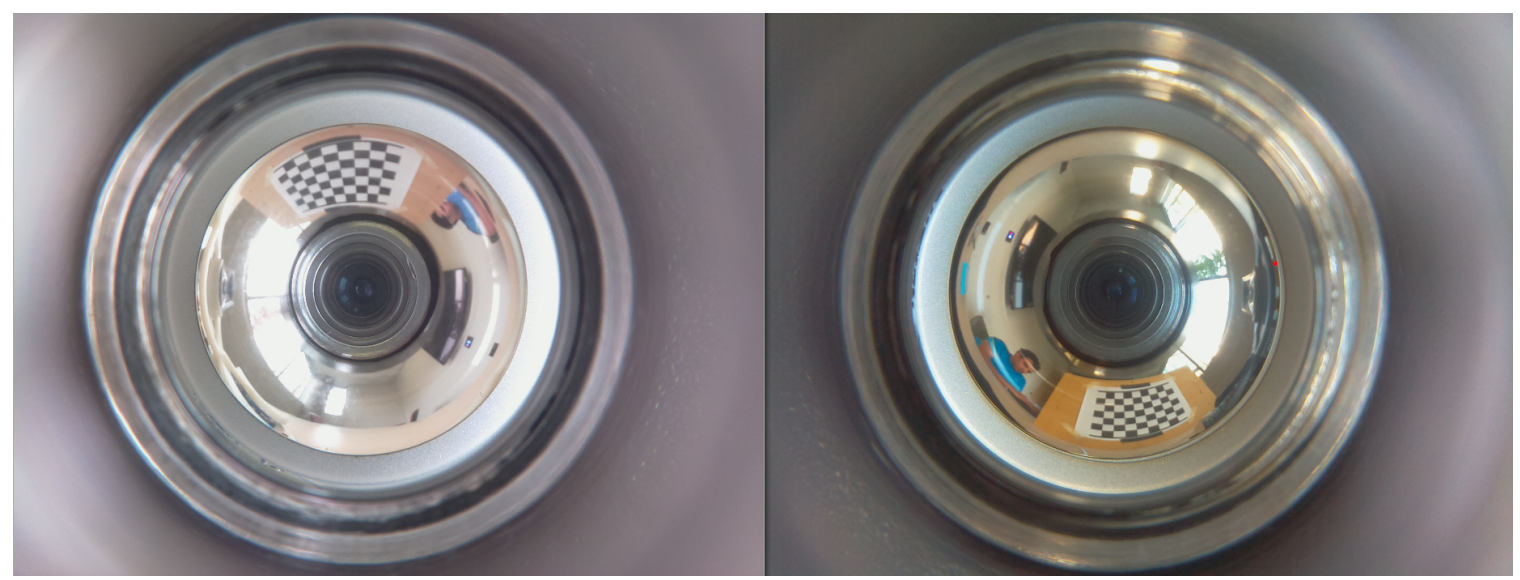

Figure 39: Effect of Applying Dark Paint on Enhancing Captured Image Quality

the detection of a feature's coordinates can reach up to $2.07 \%$ in height and $0.22 \%$ in width, i.e., 1.266 degrees on the vertical field of view and 0.8 degrees on the horizontal field of view. However, such errors have a huge effect on the calibration process that can reach a $150 \%$ error as shown in the following example, where the simulated image is known to have $\gamma=38^{\circ}, y=0, y^{\prime}=8, \theta=20$ and $b=182$.

In this analysis, a known angle $\gamma$ is assumed to be known in order to solve for the value $b$ by using Equation (29) as follows:

For vertical error effect,

$$
\begin{gathered}
\frac{\tan (38)}{\tan \left(\frac{b-(8+6)}{b-(0-6)} \times 38\right)}=\frac{1}{\cos (20)} \\
b=437.13
\end{gathered}
$$

For horizontal error effect,

$$
\begin{aligned}
\frac{\tan (38)}{\tan \left(\frac{b-(8)}{b-(0)} \times 38\right)} & =\frac{1}{\cos (20+0.8+0.8)} \\
b & =151.65
\end{aligned}
$$




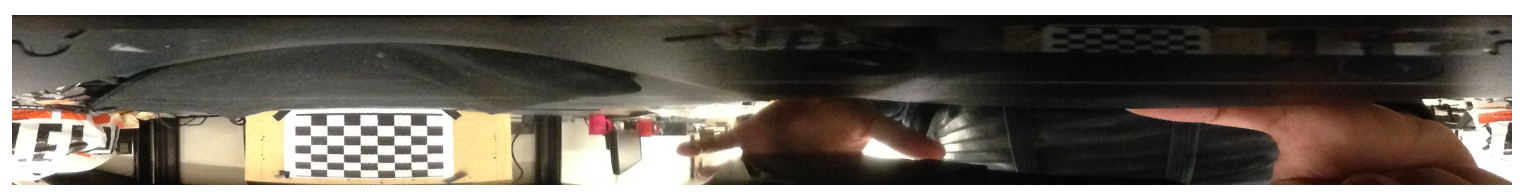

Figure 40: High Quality Image

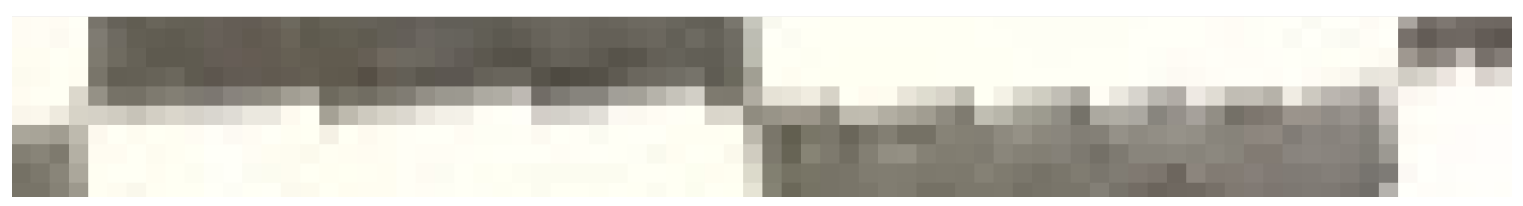

Figure 41: Found Corners From iPhone Camera

This provides an error of $140 \%$ in the identification of $b$ from the 6 pixels variation along the vertical field of view. However, the 6 pixels variation in the horizontal field of view provides an error of $16.7 \%$.

Therefore, the image calibration process is sensitive for the angular variations of the given perspectives. However, these variations does not affect the ideal epipolar geometry.

One example of a better performance camera can be shown in Figure 40. Such image is produced from a high quality iPhone 8 mega pixel camera. Moreover, a zoomed comparison between the raspberry pi 5 mega pixel image and the iPhone image can be seen in Figure 41 and Figure 42, respectively. iPhone image has proven to be of better quality with a much higher contrast. It has a maximum variation of 1 pixel along a larger unwrapped image frame that allows a significantly reduced angular errors.

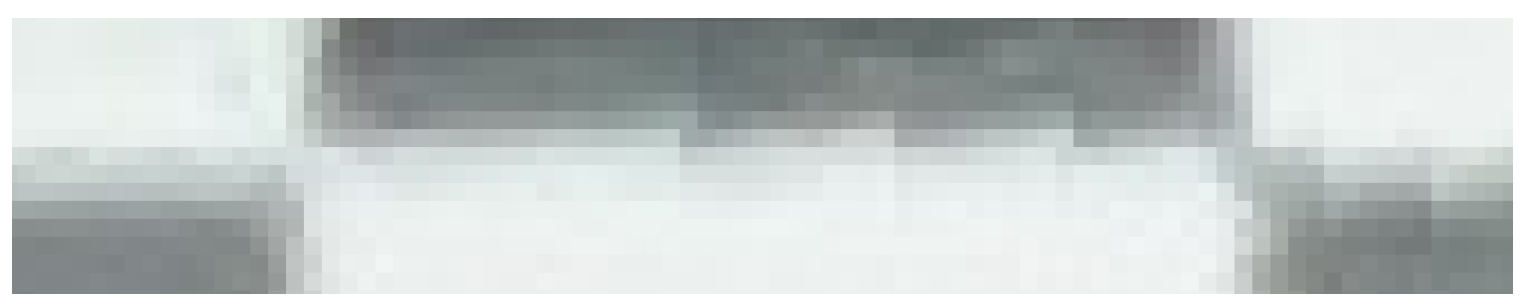

Figure 42: Found Corners From Raspberry-Pi Camera 


\subsubsection{Angular Step Size and Depth Estimation Performance}
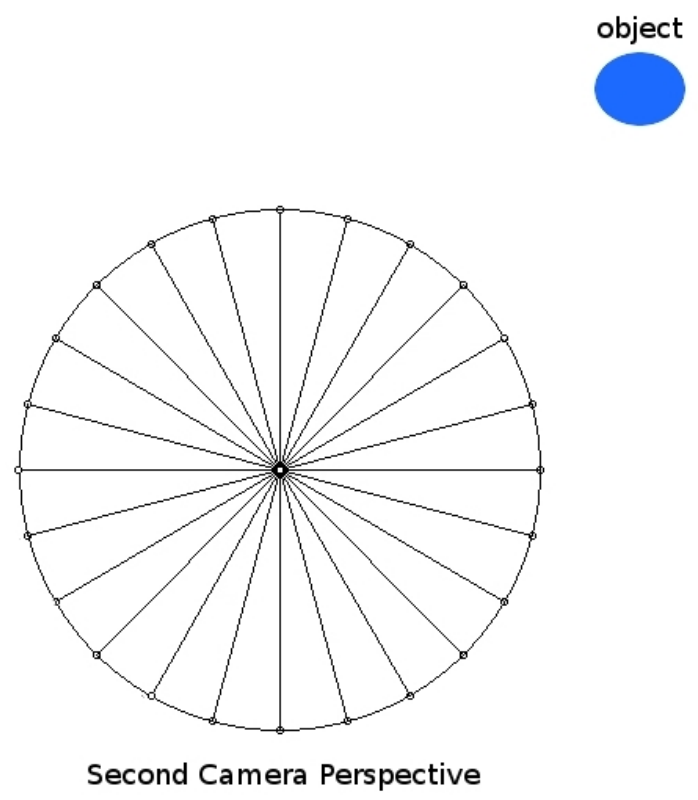

Figure 43: Angular Steps and Depth Estimation Errors

The depth estimation of the observed 3D scene features is the ultimate goal of the stereo vision systems. From the previous analysis on the side-to-side stereo panoramic vision systems, it is found that the accuracy of the depth estimation data decreases when the physical object depth increases. This happens due to the size of the angular step per pixel as shown in Equation (58) and Figure 43. Moreover, a simulation that shows the maximum applicable normal depth estimation errors is provided by using the captured 2701 column unwrapped image and a simulated 5000 column image as shown in Figure 44. This comparison illustrates the effect of the angular step size on the depth estimation quality. From Figure 44, it can be concluded that the percentage of depth estimation errors are exponentially increaseing when the physical depth of the observed feature increases. Therefore, the system's performance can be, significantly, enhanced by using larger and better quality images. Alternatively, the depth estimation quality can be improved by increasing the distance between the two 
cameras $d$ as shown in the simulations in Figure 45. In order to quantify this quality analysis, the following equation is derived for defining the maximum normal depth estimation error for features in the middle of the distance between the two cameras

$$
e=\frac{d}{2} \times \tan (\theta+S)-d e p t h
$$

where $e$ is the maximum depth estimation error, $d$ is the stereo camera's baseline, $S$ is the azimuth angular steps, and depth is the actual normal depth of the observed environment feature in the horizontal plane.

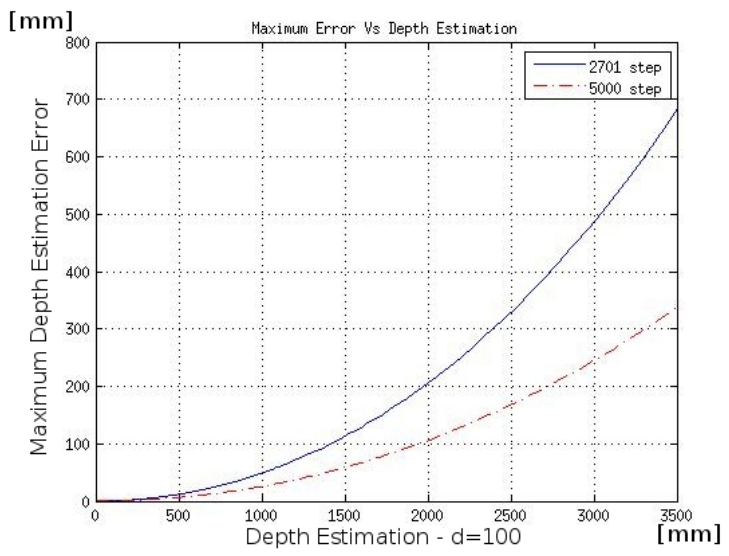

Figure 44: Maximum Depth Estimation Errors (at $d=100)$

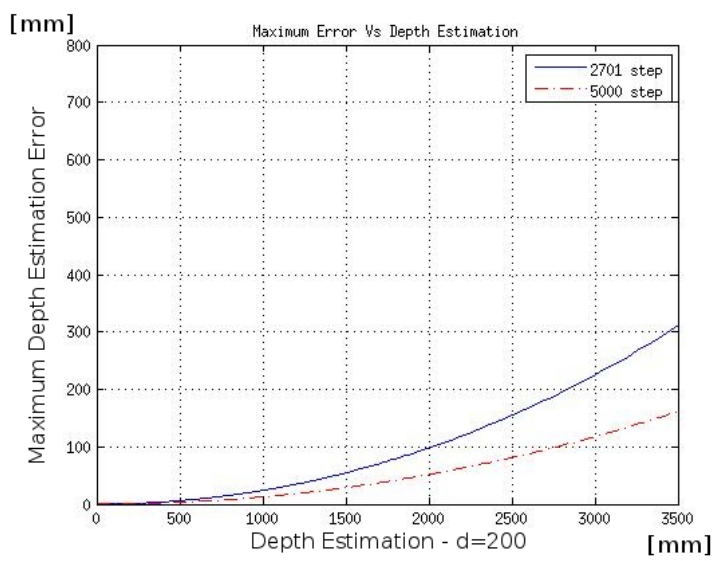

Figure 45: Maximum Depth Estimation Errors (at $d=200$ ) 


\subsubsection{Distance Measurement}

For the observation of the provided scene feature, a 3D printed experimental setup with mounted stereo panoramic cameras is used as shown in Figure 46. In this setup, the stereo cameras have a baseline $d=100 \mathrm{~mm}$. In particular, a measure tape is used with stereo panoramic images to define the physical depth and the estimated depth of a provided scene feature. In order to verify the depth estimation technique and its correspondent errors, experimental depth estimation results from different distances are provided as shown next. In particular, the horizontal normal depth is calculated using Equation (46) while the actual depth is defined by using a measure tape. Moreover, the maximum applicable error due to the angular step size is calculated using Equation (58)

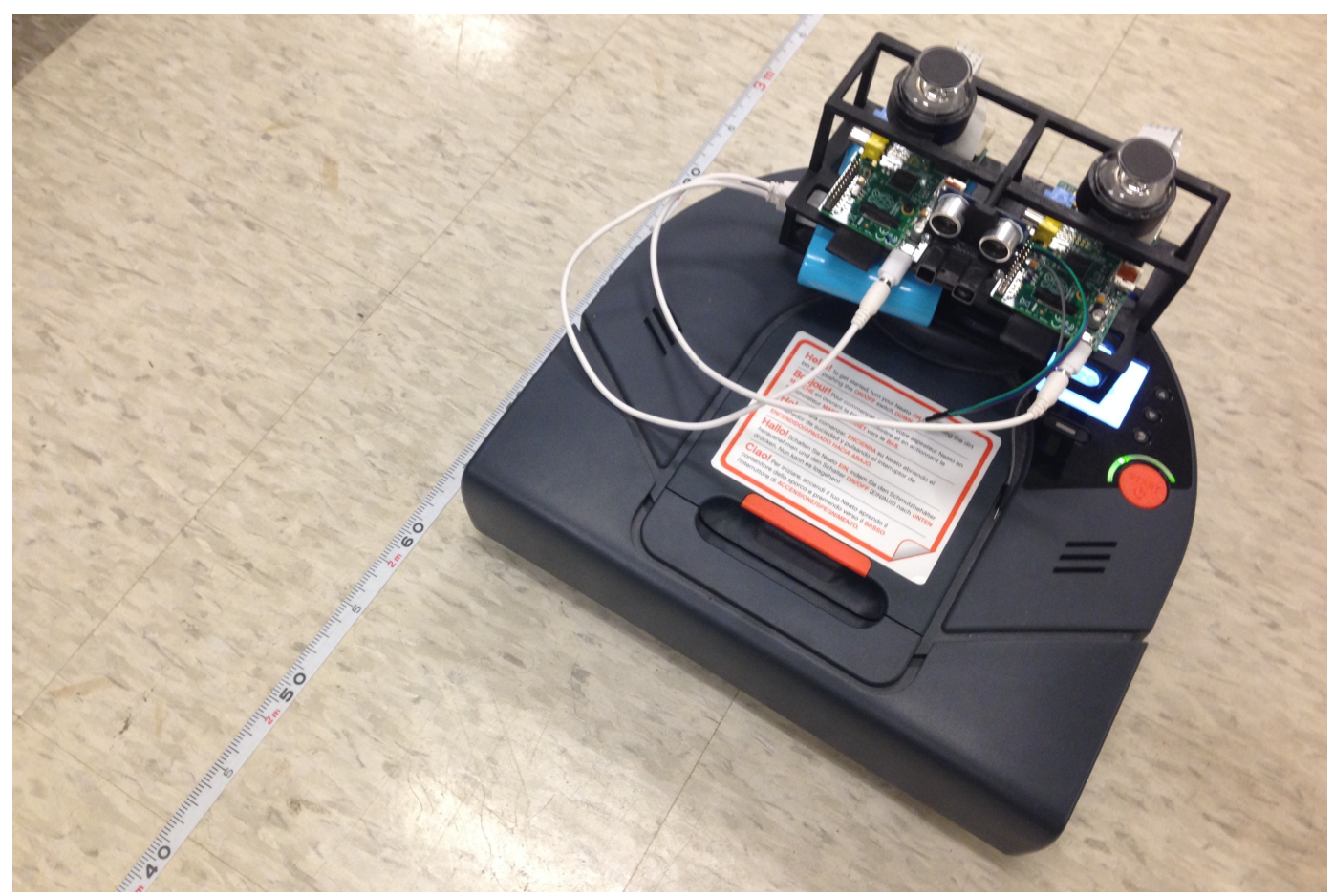

Figure 46: Defined Distance Measurement 


\section{Case A: 3.2 meters}

From the distance 3.2 meter shown in Figure 47, the panoramic scene is observed as shown in Figure 48. From the given perspectives, the 3D scene feature is found at a normal depth of $3.228 \mathrm{~m}$. This observation provides an actual error of $28 \mathrm{~mm}$ from the distance measured with the measure tape. However, the angular step size introduces a maximum depth estimation error of $559.88 \mathrm{~mm}$ for a feature observed at a physical depth of $3.2 m$, i.e., $17.5 \%$ error for one angular step variance.

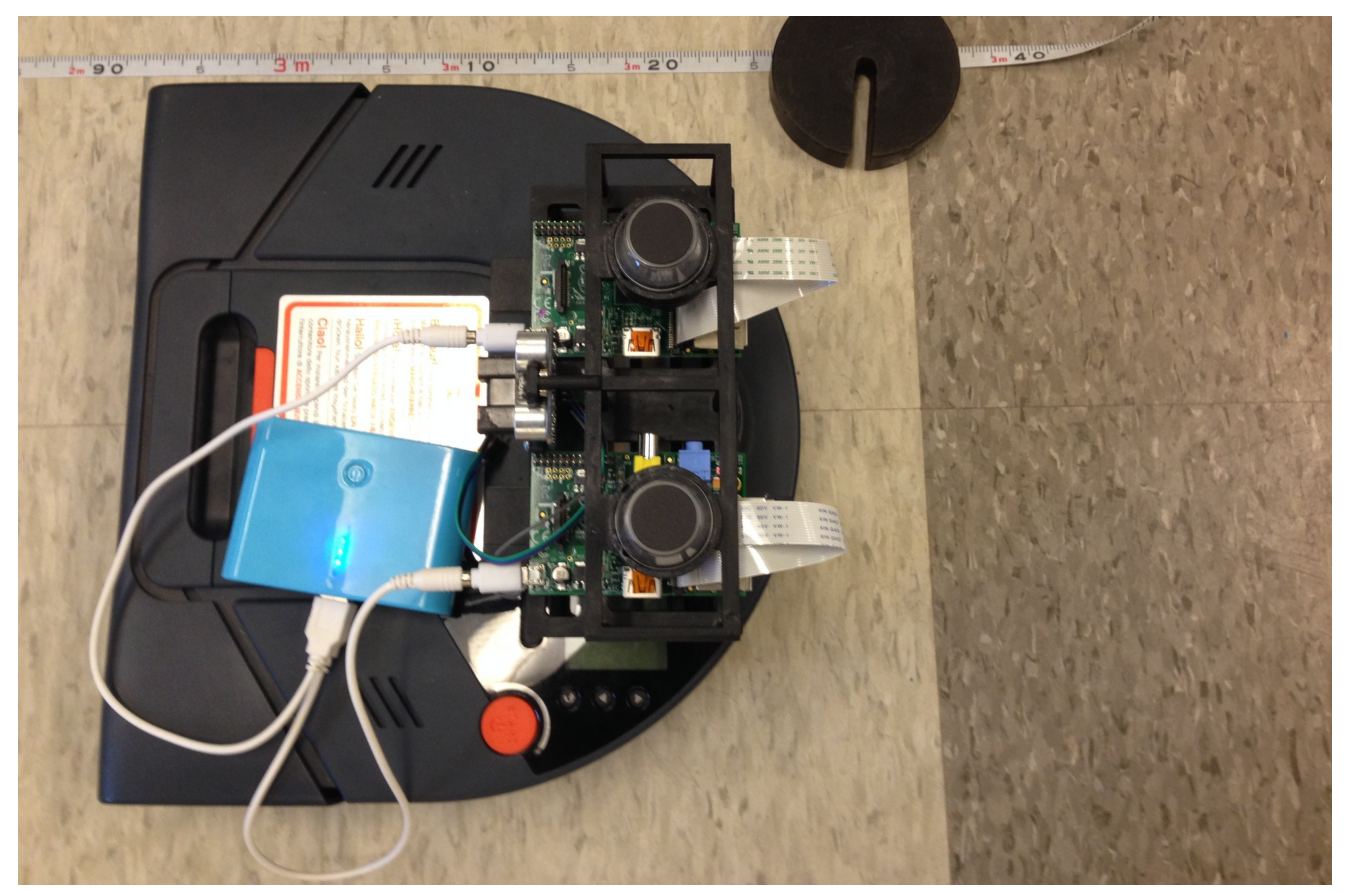

Figure 47: Robot at Distance 3.2 Meters

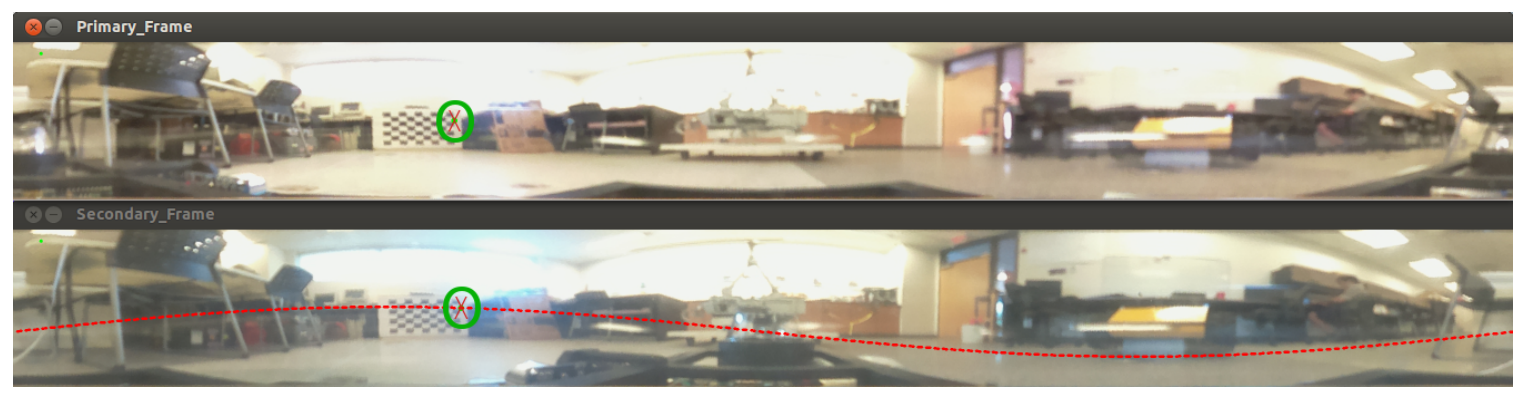

Figure 48: Epipolar Geometry Simulation - 3.2 Meters 


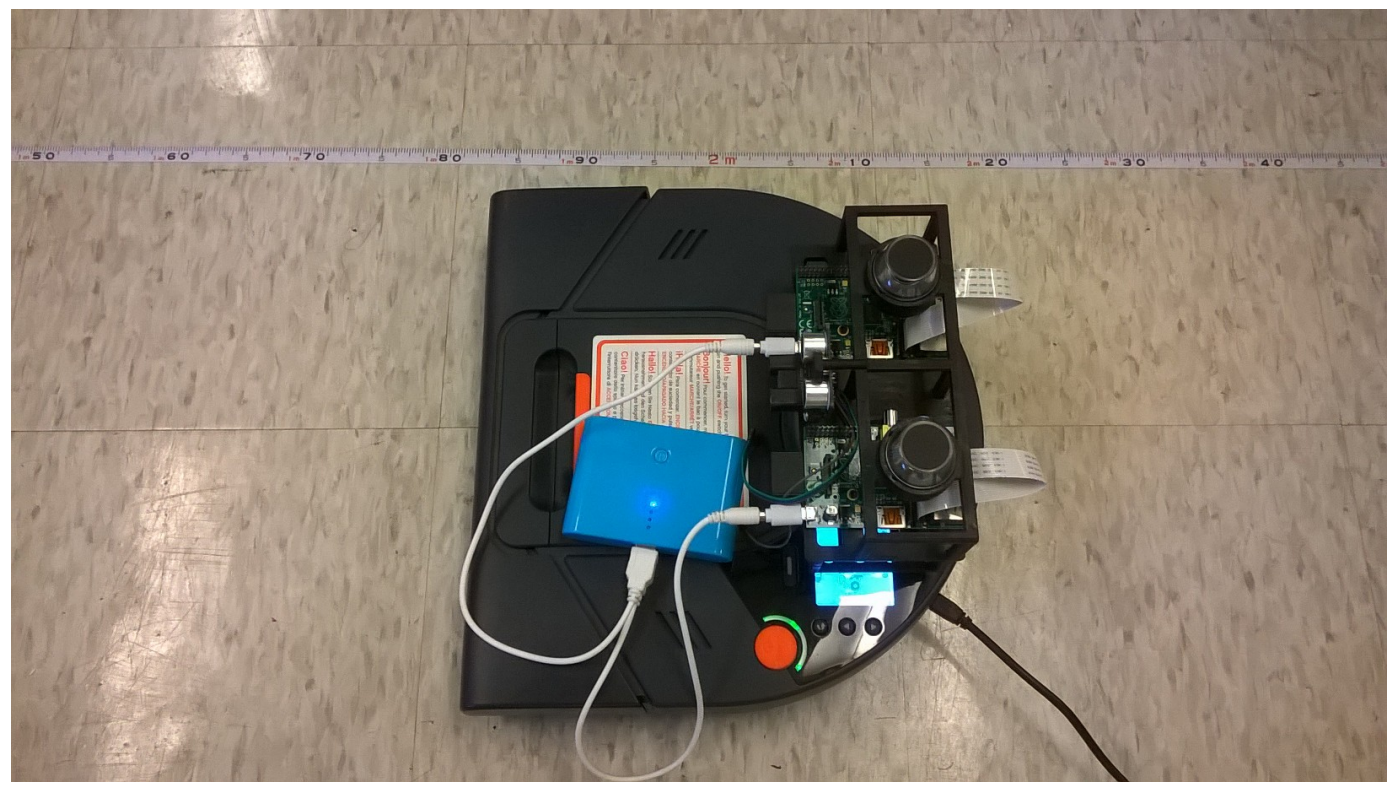

Figure 49: Robot at Distance 2.1 Meters

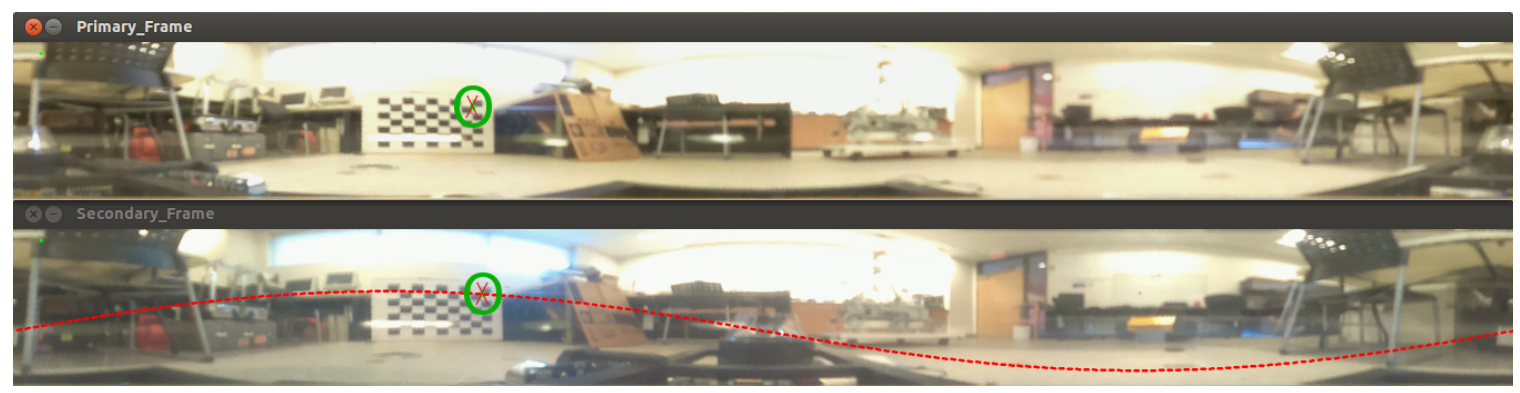

Figure 50: Epipolar Geometry Simulation - 2.1 Meters

\section{Case B: 2.1 meters}

From the distance 2.1 meters shown in Figure 49, the panoramic perspectives of the 3D scene are found as shown in Figure 50. From the provided perspectives, the $3 \mathrm{D}$ scene feature is found at a normal depth of $2.042 \mathrm{~m}$. This depth estimation has an observed error of $58 \mathrm{~mm}$ in the normal depth. However, the observation at such physical depth provides a maximum depth estimation error of $227.52 \mathrm{~mm}$, i.e., $10.83 \%$ error. 


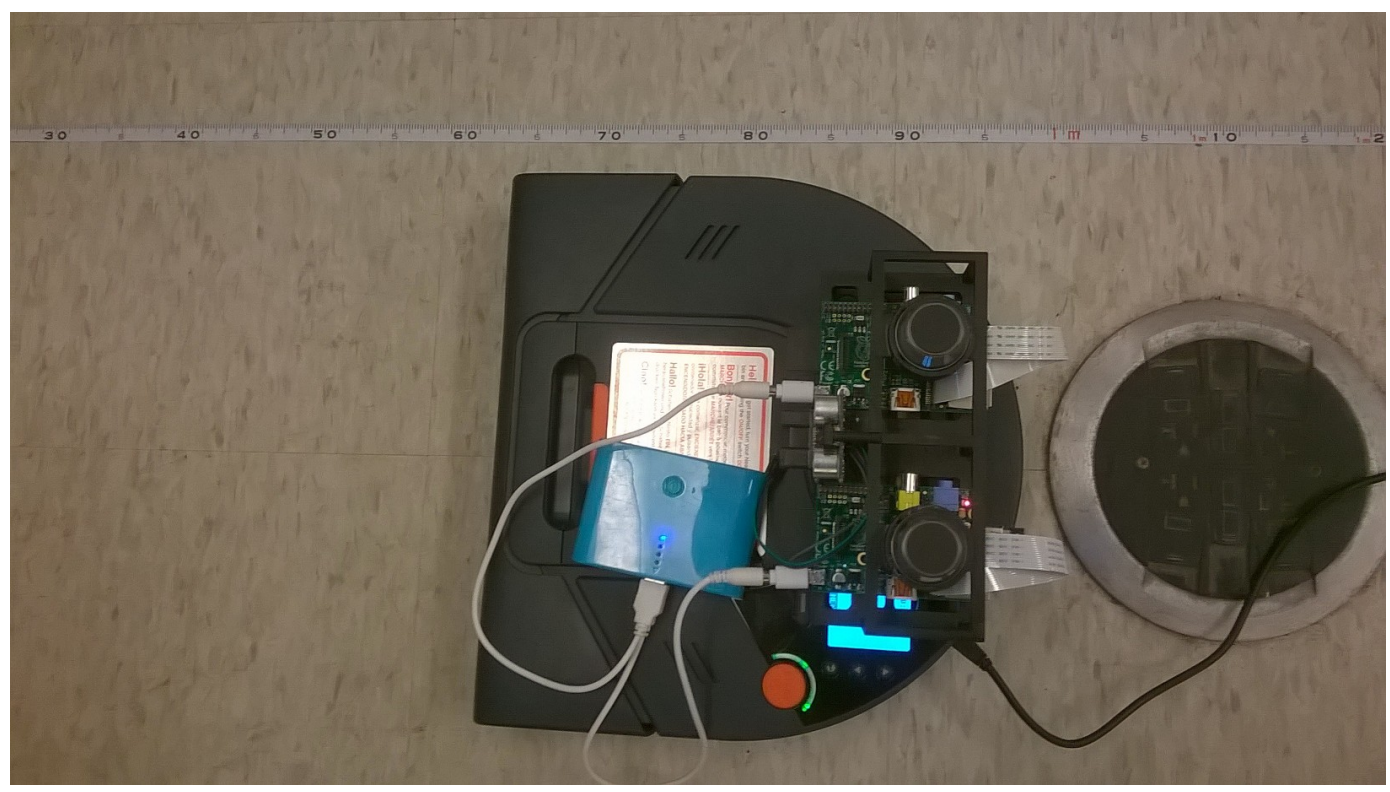

Figure 51: Robot at Distance $90 \mathrm{~cm}$

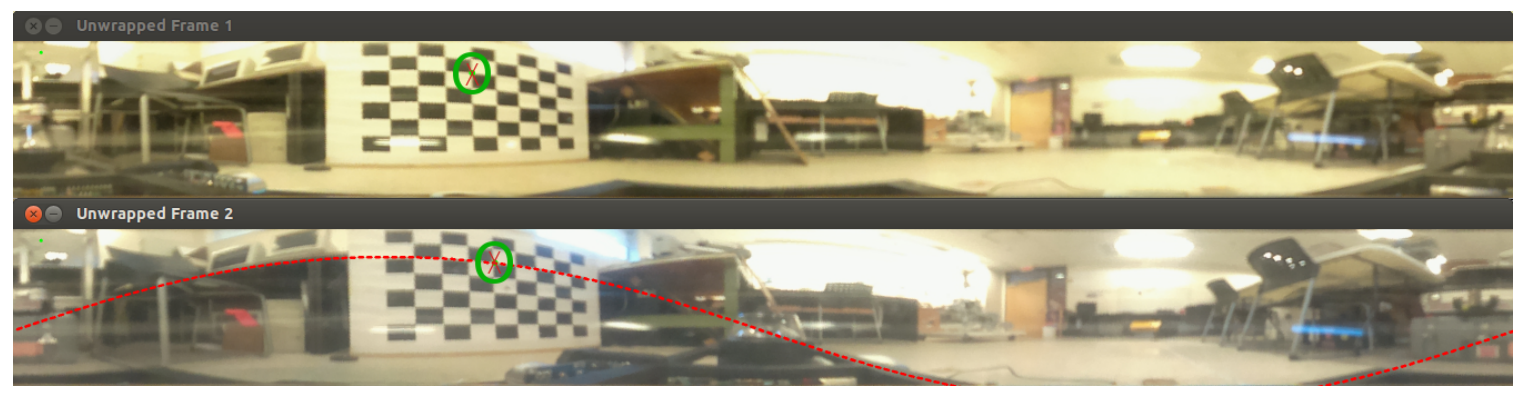

Figure 52: Epipolar Geometry Simulation - $90 \mathrm{~cm}$

\section{Case C: $90 \mathrm{~cm}$}

From the 90 centimeters distance shown in Figure 51, the 3D scene is observed as shown in Figure 52. From these given perspectives, the 3D scene feature is located at a normal depth of $90.7 \mathrm{~cm}$. This observation provides us with a depth estimation error of $7 \mathrm{~mm}$. However, the maximum depth estimation error in one step variation along the observed lens horizon is $39.453 \mathrm{~mm}$, i.e., $4.383 \%$ error. 


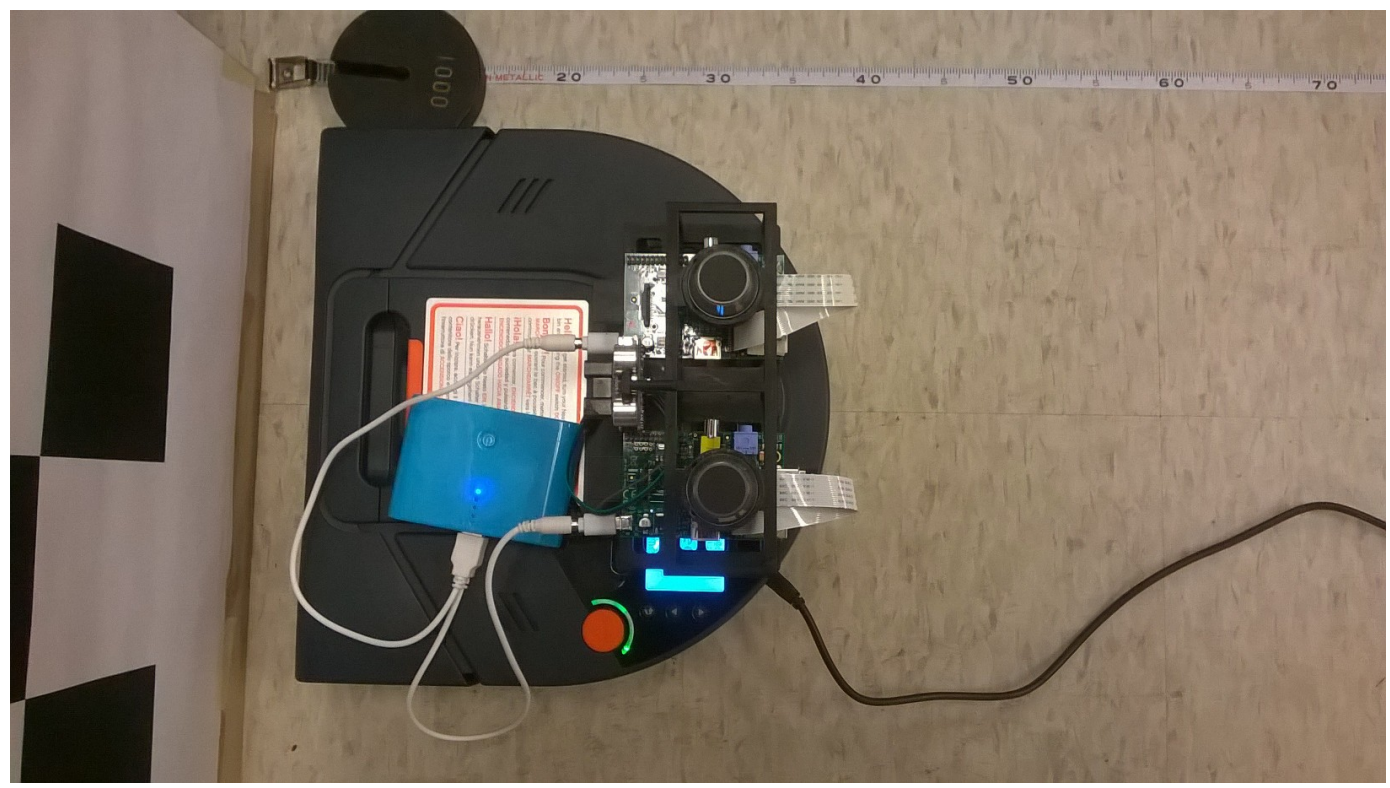

Figure 53: Robot at Distance $30 \mathrm{~cm}$

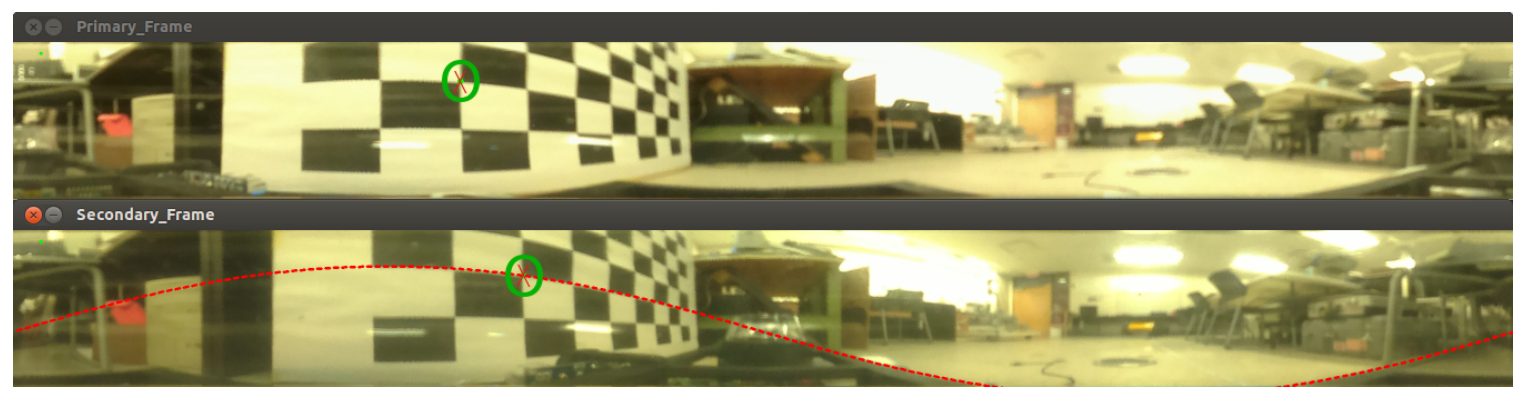

Figure 54: Epipolar Geometry Simulation - $30 \mathrm{~cm}$

\section{Case D: $30 \mathrm{~cm}$}

From the $30 \mathrm{~cm}$ distance shown in Figure 53, the panoramic scene is observed as shown in Figure 54. From these perspectives, the 3D scene feature is found to be at a normal depth of $30.135 \mathrm{~cm}$. This observation provides an actual depth estimation error of $1.35 \mathrm{~mm}$. However, one step variation along the panoramic perspective provides a maximum depth estimation error of $4.364 \mathrm{~mm}$, i.e., $1.45 \%$ error. 


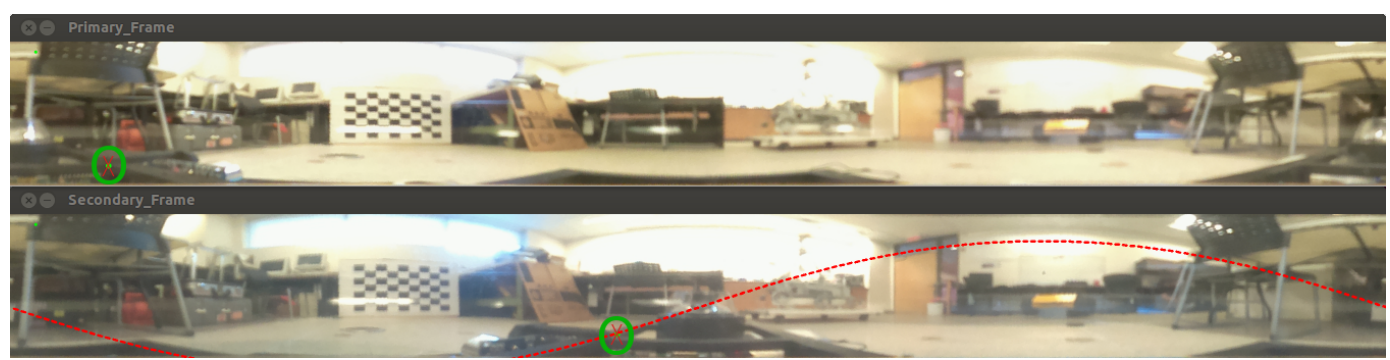

Figure 55: Epipolar Geometry Simulation - $215 \mathrm{~mm}$

\section{Case E: $2.15 \mathrm{~cm}$}

Finally, a very close image feature correspondence is tested to validate the system's depth estimations accuracy. An observable part of the 3D printed camera fixture is chosen as shown in Figure 55. This feature is measured to have a $21.5 \mathrm{~mm}$ normal physical distance from the two cameras. From this observation, the 3D scene feature is estimated to be at a normal depth of $21.52 \mathrm{~mm}$. This observation provides us with an error of $0.02 \mathrm{~mm}$. However, the maximum depth estimation error from one step variation at this physical depth is calculated to be $0.1379 \mathrm{~mm}$, i.e., $0.064 \%$ error. 


\section{Chapter 6}

\section{Summary, Future Work and Conclusions}

\subsection{Summary}

\subsubsection{Chapter 1}

In Chapter 1, an introduction about the types of mobile robots and their control architecture is provided, i.e, Unmanned Ground Vehicles (UGV). A brief explanation

on autonomous Simultaneous Localization And Mapping (SLAM) is provided. In particular, a brief discussion on the mapping methods, the localization approach, and the path planning categories. Moreover, a picture of the used mobile robot is provided with the thesis organization.

\subsubsection{Chapter 2}

Chapter 2 provides an explanation of the different types of range finding techniques, i.e., transceivers and stereo vision systems. Moreover, a detailed explanation on the conventional stereo vision systems is provided along with their calibration method, rectification approach, and correspondent epipolar geometry. 


\subsubsection{Chapter 3}

Chapter 3 introduces the advantages of omni-directional and panoramic vision systems. The different lens structures are introduced and discussed. Then, a relevant literature review on the omni-directional cameras calibration approaches. In addition, a literature review on the use of omni-directional cameras for mobile robot positioning is provided.

\subsubsection{Chapter 4}

In chapter 4, a new calibration approach for a side-to-side stereo panoramic vision system is introduced. This approach is based on using the unwrapped panoramic image frames with a specially designed calibration board. In order to validate the proposed approach, discussions supported with relevant simulations and experiments are provided in this chapter.

\subsubsection{Chapter 5}

Chapter 5 explains the different formats of stereo omni-directional vision systems. Then, an approach for defining the transformation matrix of stereo side-to-side omnidirectional cameras is provided. Moreover, a derivation for the epipolar geometry of the proposed setup is explained with relevant experiments. Finally, the effects of image distortions and panoramic image perspectives on the quality of correspondent features depth estimation are discussed with supportive simulations and experiments. 


\subsection{Future Work}

\section{Image Quality Enhancement}

The quality of the captured panoramic images directly affects the capability of detecting the features on a given image. In particular, the omni-directional images have a reduced quality due to its circular structure. Furthermore, the enhanced image quality would allow more precise and accurate feature detection and matching. Therefore, a research on enhancing the image quality is recommended to allow more robust robot localization.

\section{Processing Power Optimization}

The robots' stereo vision systems continuously capture, detects and matches the observed environment features from different perspectives during its operation. Therefore, this process consumes the robot memory and reduces its efficiency in long path operation. Therefore, the feature extraction process from the 3D observed scene must be optimized. This can be done by using the minimum number of features that is sufficient for robot localization. In the proposed vacuuming application, a low profile service robot is used, i.e, robotic vacuum cleaner. The proposed perception model of this robot is capable of capturing the ground environment information as well as the environment data at heights greater than its profile. Such information might be classified as excess data that consumes robot processing while not adding value to its mapping function. Therefore, a robust method to cluster the observed data required while being capable of choosing the correct minimum number of data.

\section{Autonomous Features Matching}

Conventional feature matching techniques use rectified images where the observed 
features are sharing the same row or column in the images of the stereo perspectives. Unlike conventional vision systems, the proposed unwrapped side-to-side panoramic stereo images may not be rectified because of their epipolar constraints, i.e., every column of pixels have a specially rectified image as discussed in chapter 5 . Thus, each pixel in the primary image has a special correspondent epipolar curve in the secondary image. Therefore, an autonomous feature matching technique along the provided epipolar curve is required.

\subsection{Conclusions}

The panoramic imaging systems have a great potential for mobile robots mapping and navigation. Unlike conventional vision systems that have a single projection, panoramic imaging systems have the capability of capturing a $360^{\circ}$ image from a single shot. This reduces the robot's possibility of losing its environment features during operation. However, the usage of panoramic images increases the calibration complexity. Therefore, a constrained calibration approach was introduced to reduce the complexity of the calibration process by reducing the number of variables. In order to use this approach, a new specially designed calibration board is introduced. In particular, the proposed calibration board has two horizontal lines added to the far ends of the conventional chessboard. These added lines help in defining the intrinsic lens parameters with high robustness as shown in Chapter 3. However, it was shown in this work that the quality of the sensor and the image blurring effects might cause unreliable image calibration. Therefore, the calibration process requires a high quality imaging sensor to provide reliable results.

For the considered low profile stereo panoramic setup, a successful epipolar geometry model for stereo unwrapped image frames was proposed. Unlike the epipolar lines of the conventional vision systems, the proposed setup was proven and experimentally 
verified to generate a sinusoidal epipolar curve along the unwrapped image frames. Furthermore, a depth estimation analysis was conducted for the proposed side-to-side panoramic imaging setup. In this analysis, different panoramic images with different sizes, i.e, different number of angular steps along the panoramic projection, were used to test the effect of the angular step size on the depth estimation quality. It was found that the angular step size has a small percentage error in a given angular perspective. However, this small percentage error in the angular perspective caused an increasing error in the depth estimation quality as shown in Chapter 5. Moreover, it was shown with simulations and experiments that depth estimation errors are exponentially proportional to the distance of the observed environment features. Finally, it was proven that the depth estimation errors for the proposed side-to-side panoramic vision system can be reduced by optimizing the stereo base line length and increasing the size and quality of the captured images. 
Appendices 


\title{
Appendix A
}

\section{Code for Unwrapping Panoramic Images and Defining the Epipolar Curves of Stereo Panoramic Images.}

\author{
\#include "opencv2/highgui/highgui.hpp" \\ \#include "opencv2/imgproc/imgproc.hpp" \\ \#include <iostream $>$ \\ \#include $<$ stdio.h $>$ \\ \#include "opencv2/features $2 \mathrm{~d} /$ features $2 \mathrm{~d}$. hpp" \\ \#include "opencv2/nonfree/features $2 \mathrm{~d}$.hpp" \\ \#include "opencv2/nonfree/nonfree.hpp" \\ \#include "opencv2/features $2 \mathrm{~d} /$ features $2 \mathrm{~d}$. hpp" \\ \#include "opencv2/core/core.hpp" \\ \#include "opencv2/opencv.hpp" \\ \#include $<$ time. $\mathrm{h}>$
}

\#define pi 3.14159265358979323846 


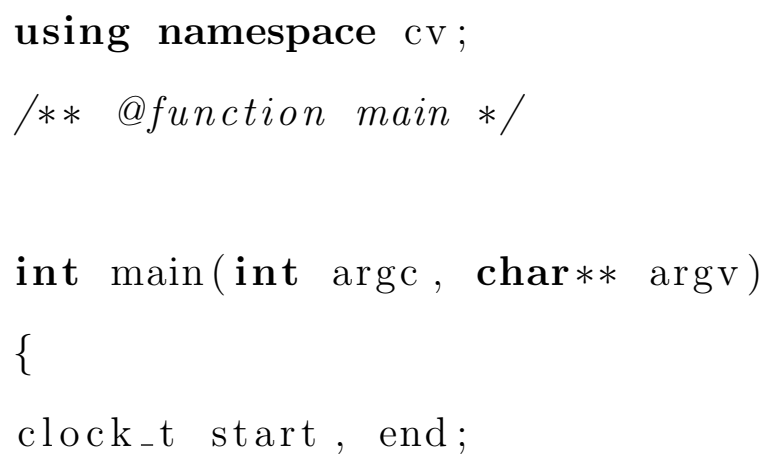

Mat src, uw, gray, edges, src_2 $_{-}, \mathrm{uw}_{-} 2$, gray_2, edges_2, can , uw_i,$\quad \mathrm{uw}_{-} 2{ }_{-} \mathrm{i}$;

int lowThreshold $=10$;

$\operatorname{src}=\operatorname{imread}(\operatorname{argv}[1]$, CVLOAD_IMAGE_COLOR $)$;

// Read the file

int $\mathrm{x}=\operatorname{atoi}(\operatorname{argv}[5])$;

if (! src. data )

// Check for invalid input

\{

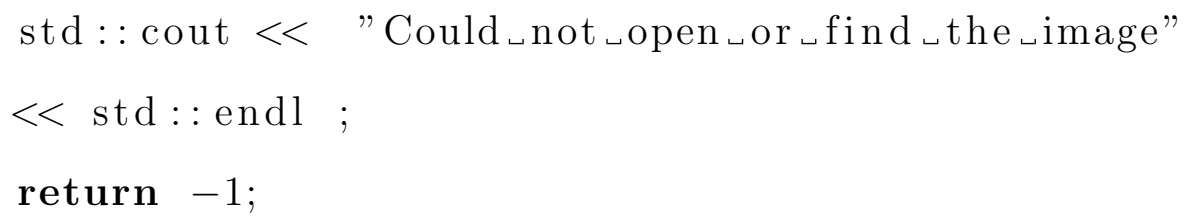


waitKey ( 0$)$;

//First Image

$$
\begin{aligned}
& \text { int } \mathrm{Pix}_{-} \mathrm{x}=1942 ; / / \operatorname{ato}(\operatorname{argv}[1]) ; \\
& \text { int } \mathrm{Pix}_{-} \mathrm{y}=50 ; / / \operatorname{atoi}(\operatorname{argv}[2]) ; \\
& \text { int } \mathrm{Pix}_{-} \mathrm{x} 1=50 ; / / \operatorname{ato} i(\operatorname{argv}[1]) ; \\
& \text { int } \mathrm{Pix}_{-} \mathrm{y} 1=20 ; / / \operatorname{ato} i(\operatorname{argv}[2]) ; \\
& \text { int Rmin, Rmax; }
\end{aligned}
$$

///Second Image

$$
\begin{aligned}
& \text { int } \mathrm{Pix}_{-} \mathrm{x}_{-} 2=50 ; \\
& \text { int } \mathrm{Pix}_{-} \mathrm{y}_{-} 2=20 ; \\
& \text { int } \mathrm{Pix}_{-} \mathrm{x}_{-} 2=50 ; \\
& \text { int } \mathrm{Pix}_{-} \mathrm{y}_{-} 2=20 ; \\
& \text { int } \mathrm{cx}, \mathrm{cy}, \mathrm{cx}_{-} 2, \mathrm{cy}_{-} 2 \\
& \text { int }
\end{aligned}
$$

///Intrinsic Camera Parameters

$$
\text { if }(\mathrm{x}==1080)
$$

\{

$$
\begin{aligned}
& \mathrm{cx}=889 ; \\
& \mathrm{cy}=528 ; \\
& \mathrm{cx}_{-} 2=871 ;
\end{aligned}
$$




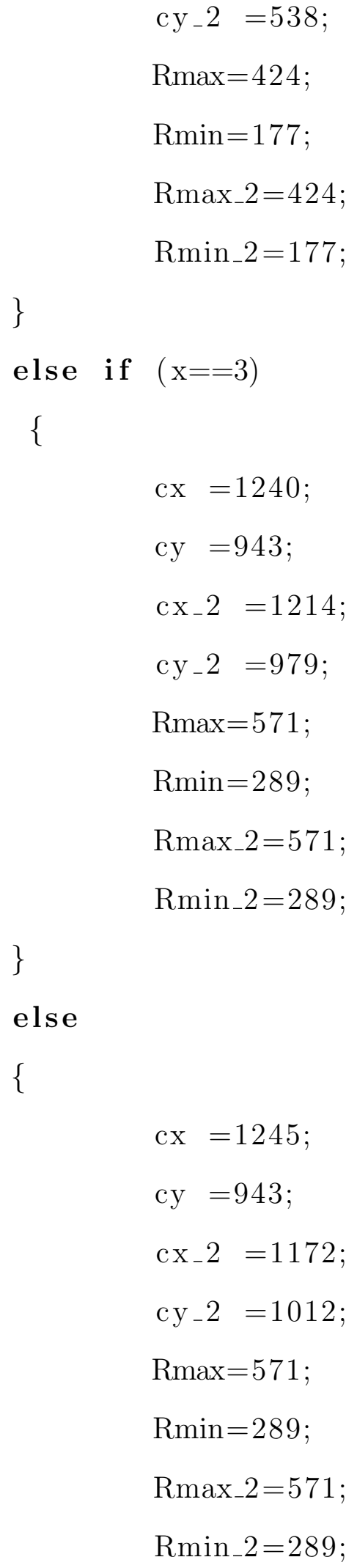


Point sq [4];

int $\mathrm{sqz}[4]$;

int $\mathrm{F}=38$;

int $R_{-}$center $=183$;

int $\mathrm{D}=100$;

int $\mathrm{sec}_{-}$theta $=286$;

int $\mathrm{DEPTH} 2=206$;

int $\mathrm{THETA} 1=66$;

int Hight $=115$;

\\Code Start

for $(; ;)$

\{

start $=\operatorname{clock}()$

int $\mathrm{k}$;

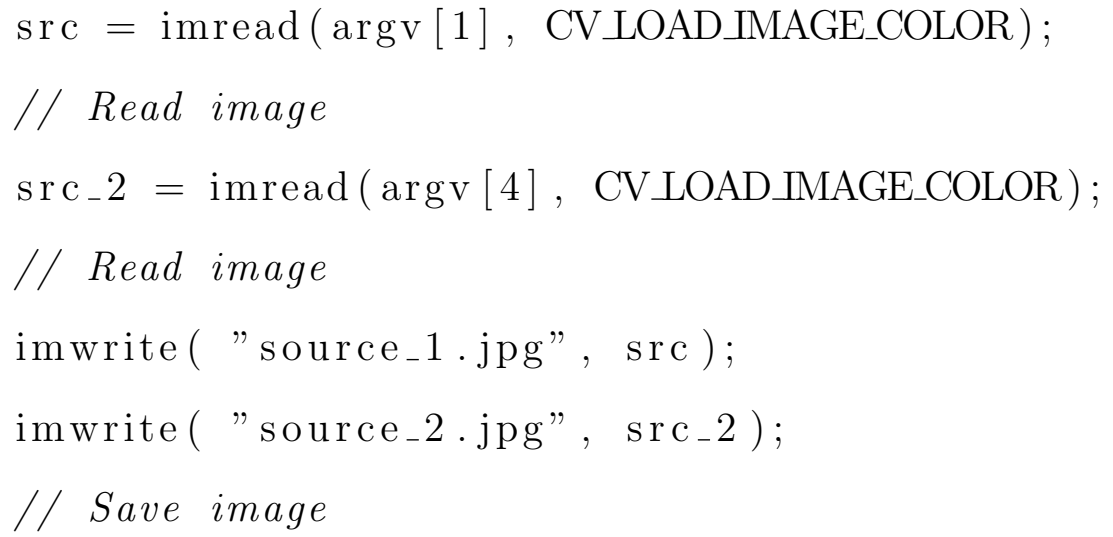


namedWindow ("Myчtrackbars", 1);

// Create a trackbar window

\section{//Points Used For Depth Estimation}

createTrackbar ("Second ${ }$ Theta", "My॰trackbars", \&sec_theta , 360 ); createTrackbar ("DEPTH2" , "My॰trackbars" , \&DEPTH2, 3000$)$; createTrackbar ("THETA1" , "Myьtrackbars" , \&THETA1, 360 ); createTrackbar ("HIGHT", "My॰trackbars" , \&Hight , 500);

\section{//First Image}

createTrackbar ("cx", "Myьtrackbars" , \&cx, 5000); createTrackbar ("cy", "Myьtrackbars", \&cy, 3000); createTrackbar ("Rmin" , "My॰trackbars" , \&Rmin, 5000); createTrackbar ("Rmax" , "My॰trackbars" , \&Rmax, 5000 ); createTrackbar ("pix_x", "My॰trackbars", \&Pix_x , 5000); createTrackbar ("pix_y", "My॰trackbars" , \&Pix_y , 400); createTrackbar ("pix_x1", "My_trackbars", \&Pix_x1, 5000); createTrackbar ("pix_y1", "My॰trackbars" , \&Pix_y1 , 5000); createTrackbar ("image center" , "My॰trackbars" , \&R_center , 3000);

\section{///Second Image}

createTrackbar ("cx_2", "My॰trackbars" , \&cx_2, 5000); createTrackbar ("cy_2", "My॰trackbars" , \&cy_2 , 3000); createTrackbar ("Rmin_2" , "My॰trackbars" , \&Rmin_2, 5000); createTrackbar ("Rmax_2" , "My_trackbars" , \&Rmax_2 , 5000 ); createTrackbar ("pix_- _ 2", "My॰trackbars", \&Pix_x_2, 5000$)$; 
createTrackbar ("pix_y_2" , "My॰trackbars" , \&Pix_y_2, 5000$)$; createTrackbar ("pix_x $1_{-} 2 "$, , "My॰trackbars" , \&Pix_x1_2, 5000); createTrackbar ("pix_y $1_{-} 2 "$, "My॰trackbars" , \&Pix_y $\left.1_{-} 2,5000\right)$; createTrackbar ("X", "My॰trackbars", \&sq[1].x, 500); createTrackbar ("Y", "My॰trackbars", \&sq[1].y, 500); createTrackbar ("Z" , "Myьtrackbars", \&sqz [1]，500); createTrackbar ("Field of $_{\llcorner}$View" , "My॰trackbars" , \&F,90); createTrackbar ( "Min Threshold:" , "Myıtrackbars" , \&lowThreshold, 100 );

Point pt( Pix_x, Pix_y $)$;

Point pt1(Pix_x1, Pix_y1);

Point pt_2( $\left.\mathrm{Pix}_{-} \mathrm{x}_{-} 2, \mathrm{Pix}_{-} \mathrm{y}_{-} 2\right)$;

Point pt $1_{-} 2\left(\mathrm{Pix}_{-} \mathrm{x} 1_{-} 2, \mathrm{Pix}-\mathrm{y} 1_{-} 2\right)$;

\section{/// IMAGE UNWRAPPING}

int Length $=2 * \mathrm{pi} *(\operatorname{Rmax}+\mathrm{Rmin}) / 2$;

int Length_2 $=2 *$ pi $*\left(\operatorname{Rmax}_{-} 2+\mathrm{Rmin} \_2\right) / 2$;

int Width= Rmax-Rmin;

int Width_2=Rmax_2-Rmin_2 ;

uw. create ( cv: : Size( (Length), (Width) ), src.type () ); uw_2. create ( cv::Size ( (Length_2), (Width_2) ), src_2.type ()$)$;

double a, r, iX, iY, R, theta;

for $(a=0 ; a<$ Length; $a++)$ 


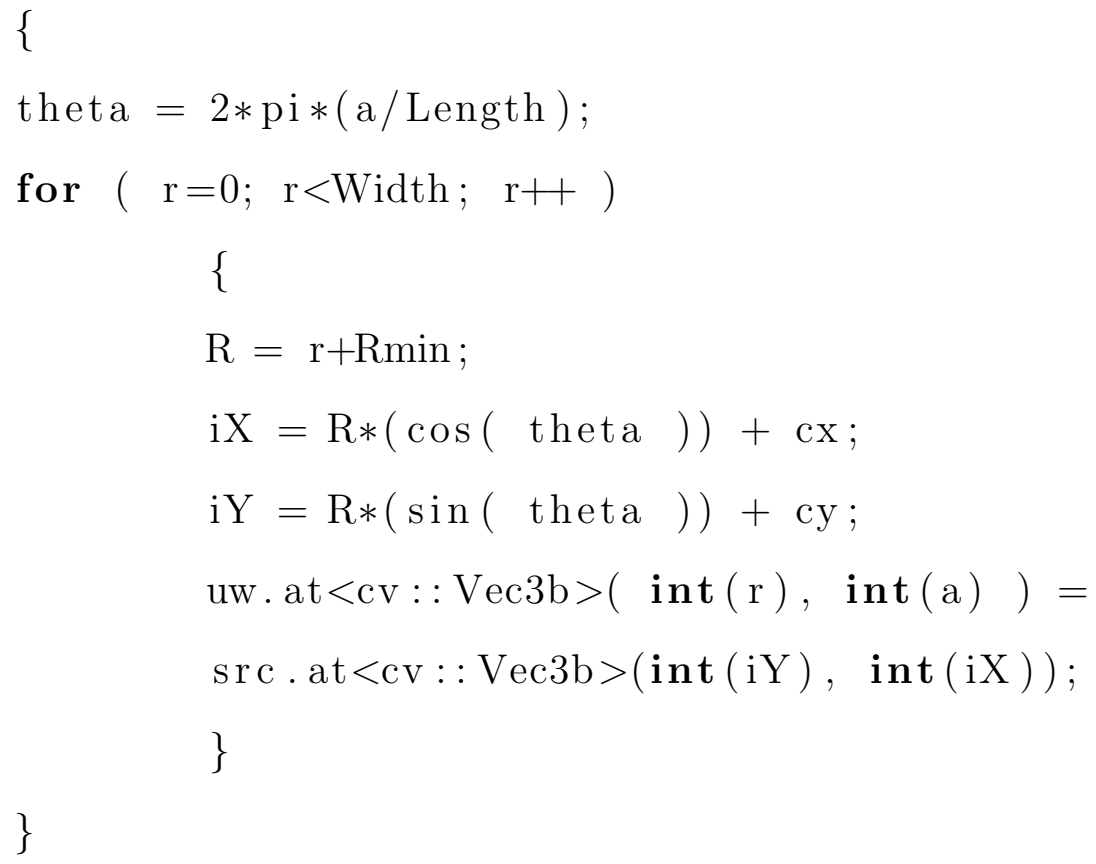

for $(a=0 ; a<$ Length_2; $a++)$

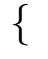




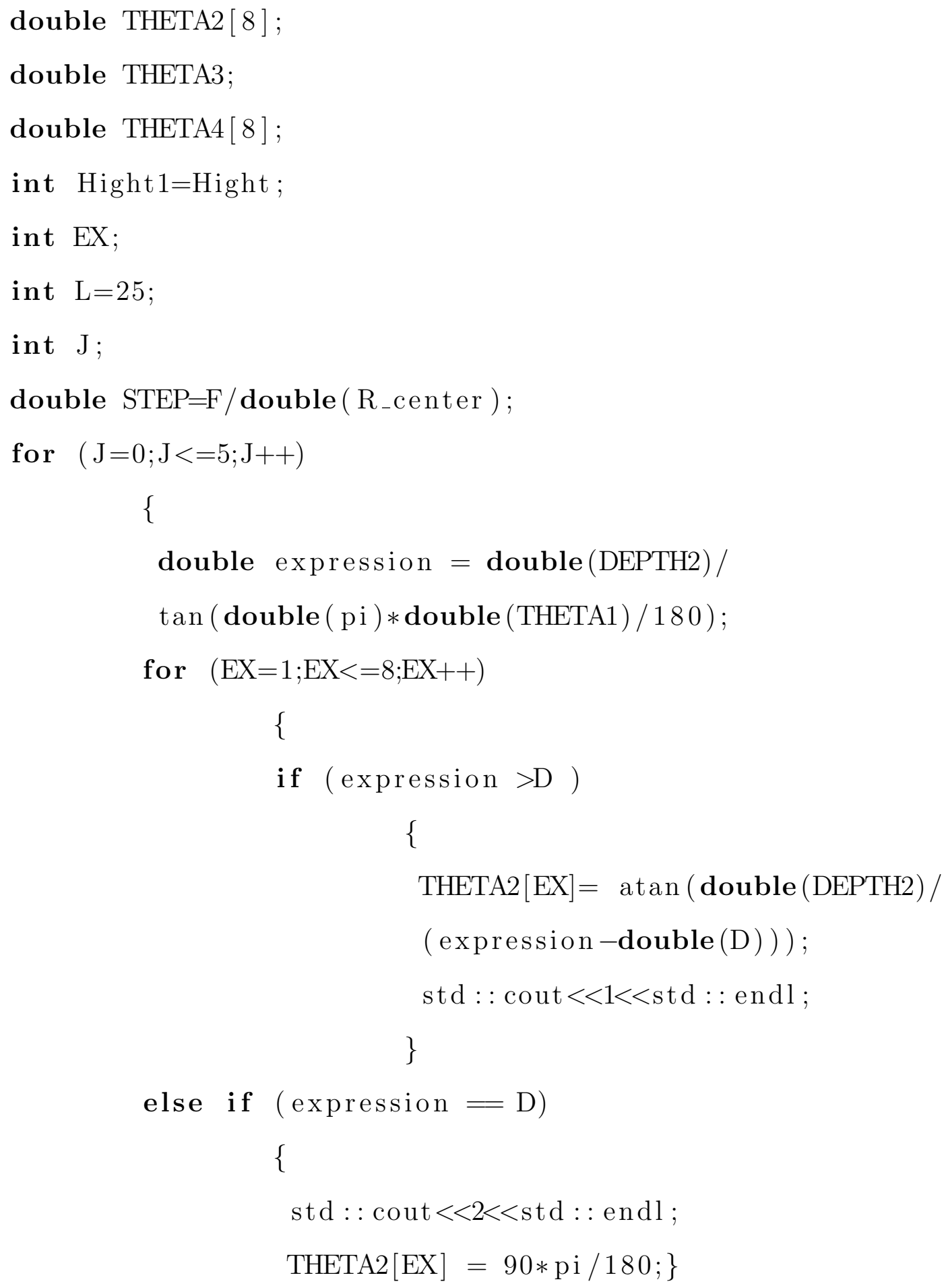


else

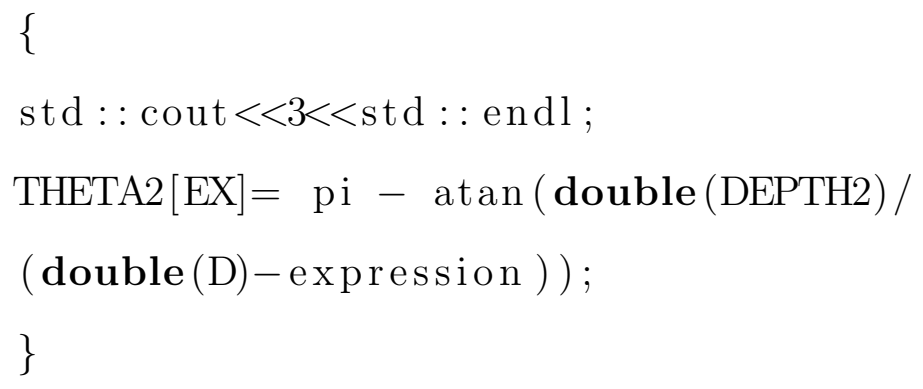

double $\mathrm{X}_{-}$position;

if $(\operatorname{atan}($ double $($ DEPTH2 $) /$ expression $)<0)$

$\mathrm{X}_{-}$position $=$

$($ pi $+\operatorname{atan}($ double $($ DEPTH2) / expression $)) *$

Length / $(2 *$ pi $)$;

else

$\mathrm{X}_{-}$position=atan $(\boldsymbol{d o u b l e}(\mathrm{DEPTH} 2) / \operatorname{expression})$

*Length / $(2 *$ pi $)$;

double HYP =

DEPTH2/sin ( $\mathrm{X}_{\text {_position }} * 2 * \mathrm{pi} /$ Length $)$;

double Y_position $=$

R_center-atan (Hight1/HYP) / (STEP*pi / 180);

double HYP2 =

double (DEPTH2) / s in (THETA2 [EX]) ;

double $\mathrm{X}_{\text {-position } 2}=$

THETA2 $[\mathrm{EX}] *$ Length $/(2 * \mathrm{pi})$;

double $\mathrm{Y}_{-}$position $2=$

R_center-atan (Hight1/HYP2)/(STEP*pi / 180);

Point PT3( X_position, Y_position ); 
Point PT4( $\mathrm{X}_{-}$position2, Y_position 2);

std : : cout $<<$ "Point1 $\lrcorner "<<$ EX $<<"$, ," $<<$ J

$<$ "

$\mathrm{Y}_{-}$position $<<$std : : endl;

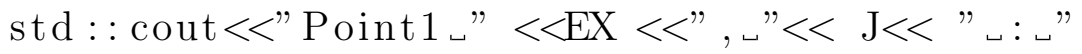

$<\mathrm{X}_{-}$position $2<", "<<\mathrm{Y}_{-}$position 2

$<<$ std : : endl ;

circle (uw, PT3, 3, cv: : Scalar $(255,0,0), 3,8,0)$;

circle (uw_2, PT4, 3, cv: : Scalar $(255,0,0), 3,8,0)$;

expression $=$ expression $-\mathrm{L}$;

\}

Hight1=Hight1 - 25;

\}

imwrite ( "Unwrapped_1.jpg" , uw );

imwrite ( "Unwrapped_2.jpg", uw_2 );

resize (uw, uw_i, Size ( ), 0.5, 0.5, INTER_LINEAR);

resize ( uw_2, uw_2_i , Size ()$, 0.5,0.5$, INTER_LINEAR);

imshow ( "unwrapped_1", uw_i );

imshow ( "unwrapped_2", uw_2_i );

///Chosen Points Of The 3D Scene Perspectives

circle ( uw, pt, 3, cv: : Scalar $(0,255,0),-1,8,0$ );

resize (src, src, Size ()$, 0.25,0.25$, INTER_LINEAR);

//Second Image Chosen Point

circle ( uw_2, pt_2, 3, cv: : Scalar $(0,255,0),-1,8,0)$; 
circle( uw, pt_2, 3, cv:: Scalar $(0,255,0),-1,8,0)$;

resize $\left(\operatorname{src} \_2_{2}\right.$, src_2 $_{2}, \operatorname{Size}(), 0.25,0.25$, INTER_LINEAR $)$;

imshow ( "source_1.jpg", src);

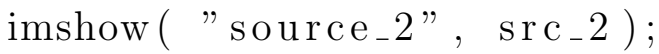

///EPIPOLAR GEOMETRY

double th1, th3;

int $\operatorname{th} 2{ }_{-} 0$;

double th $2[360]=\{\} ;$

double th $4[360]=\{\}$;

th $1=\left(\left(\operatorname{Pix}_{-} \mathrm{x} /(2 * \mathrm{pi} *(\operatorname{Rmax}+\mathrm{Rmin}) / 2)\right) * 2 * \mathrm{pi}\right) ;$

th $3=(\operatorname{pi} / 180) *\left(\operatorname{double}(\mathrm{F}) *\left(1-\left(\operatorname{double}\left(\mathrm{Pix}_{-} \mathrm{y}\right) /\right.\right.\right.$ double $\left(\mathrm{R}_{-}\right.$center $\left.\left.\left.)\right)\right)\right)$;

Point P1[360],P[360];

for $\left(\operatorname{th} 2 \_0=1 ;\right.$ th $2 \_0<361 ;$ th $\left.2 \_0++\right)$

\{

$\operatorname{th} 2\left[\operatorname{th} 2_{-} 0\right]=$ pi $-\left(\operatorname{th} 2 \_0 *(\operatorname{pi} / 180)\right)$;

$\operatorname{th} 4\left[\operatorname{th} 2_{-} 0\right]=\operatorname{atan}\left(\left(\sin \left(\operatorname{th} 2\left[\operatorname{th} 2_{-} 0\right]\right) * \tan (\operatorname{th} 3)\right) / \sin (\operatorname{th} 1)\right) ;$

$\mathrm{P} 1\left[\operatorname{th} 2{ }_{-} 0\right] \cdot \mathrm{x}=\left(\left(\mathrm{pi}-\operatorname{th} 2\left[\operatorname{th} 2 \_0\right]\right) /(2 * \mathrm{pi})\right) *(2 * \mathrm{pi} *(\mathrm{Rmax}+\mathrm{Rmin}) / 2)$;

$\mathrm{P} 1\left[\operatorname{th} 2_{-} 0\right] \cdot \mathrm{y}=\left(\left(\operatorname{double}(\mathrm{F})-\operatorname{th} 4\left[\operatorname{th} 2_{-} 0\right] *(180 / \mathrm{pi})\right) / \operatorname{double}(\mathrm{F})\right)$

$*$ double(R_center) ;

\}

int $\mathrm{X}_{-}$pos, $\mathrm{Y}_{-}$pos;

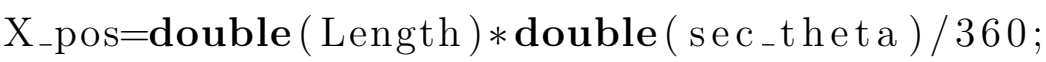

$\mathrm{Y}_{-}$pos $=\mathrm{P} 1\left[\right.$ sec_theta $_{-} \cdot \mathrm{y}$; 


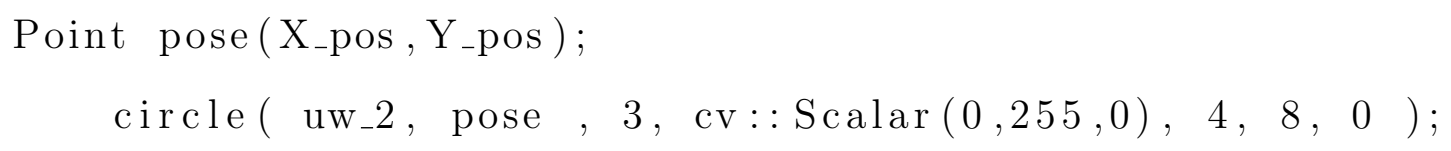

// /DEPTH ESTIMATION

double depth;

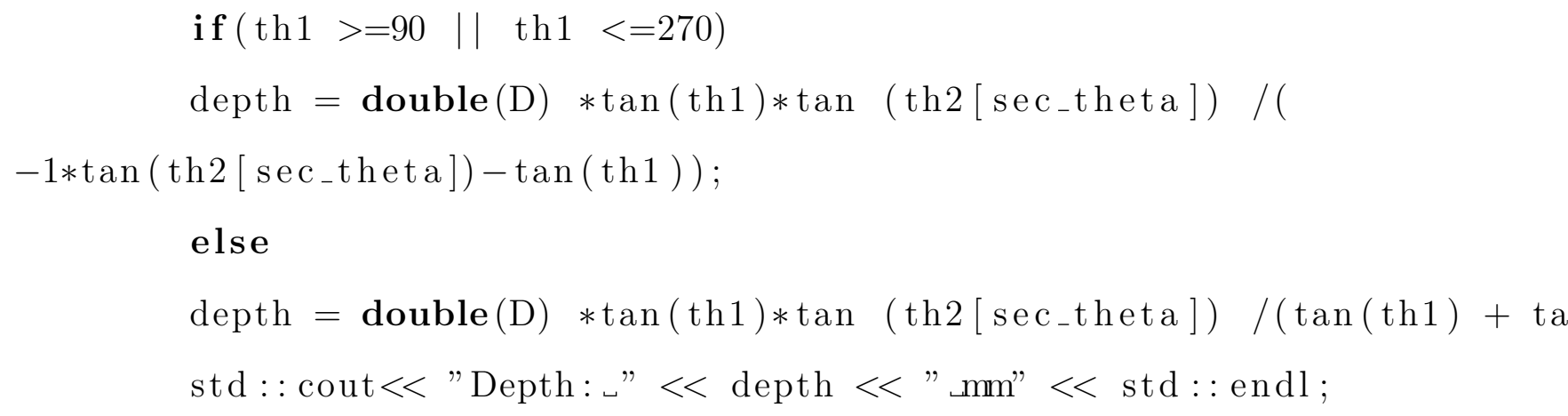




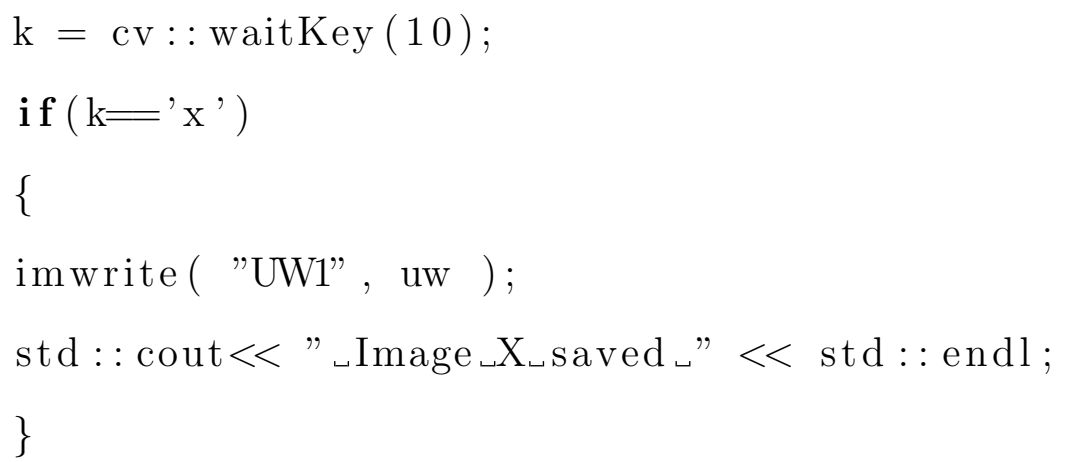

// /CORNERS DETECTION

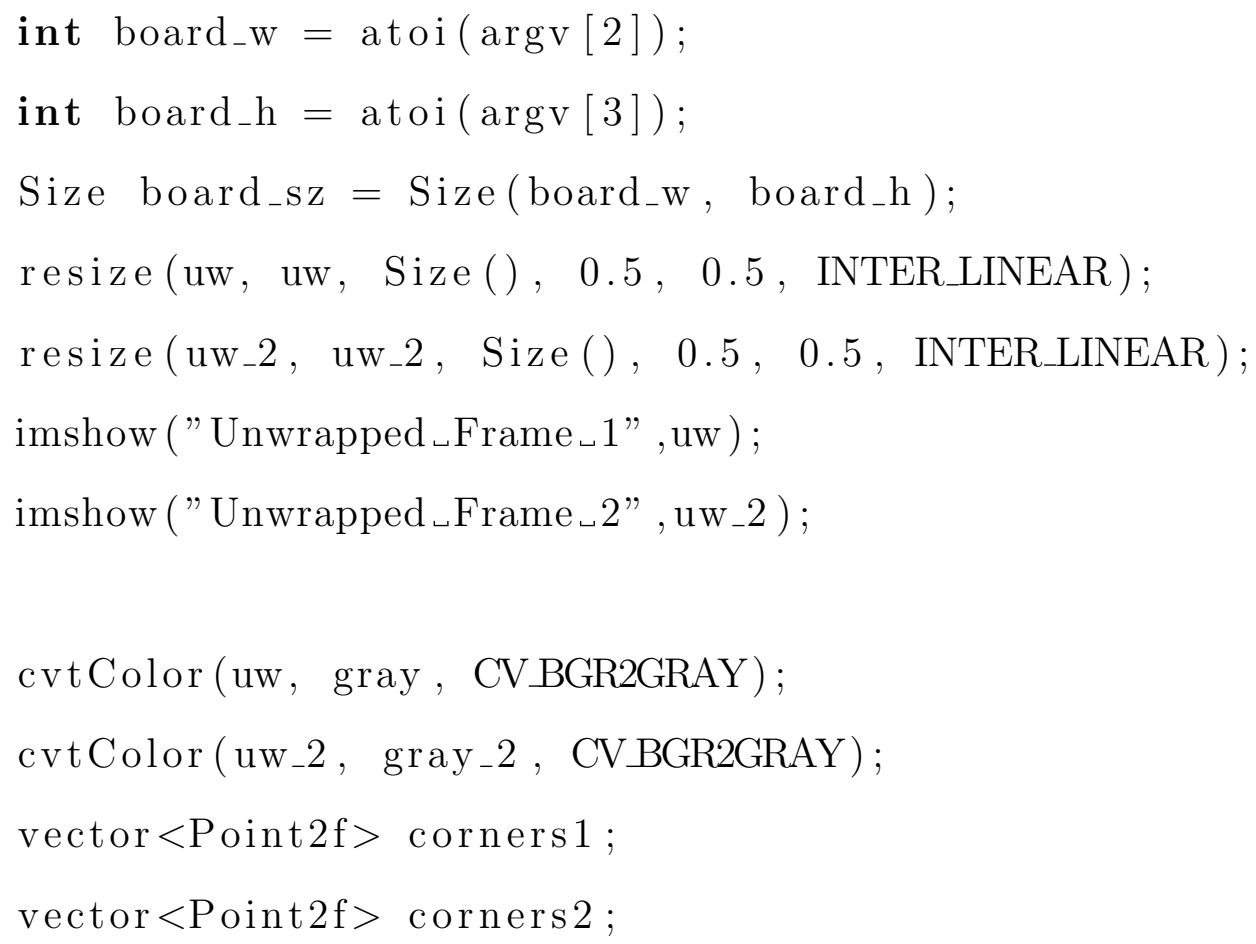


imshow("can", can); // Show canny edges for reference

found 1 = findChessboardCorners (uw, board_sz, corners1, CV_CALIB_CB_ADAPTIVE_THRESH + CV_CALIB_CB_FILTER_QUADS );

std : : cout $<<"{ }_{\sqcup}$ found ${ }_{\llcorner}$corners f $"<<$ corners $1<<$ std: : endl; //Print found corners drawChessboardCorners (gray, board_sz , corners1, found1); drawChessboardCorners (uw, board_sz, corners1, found1);

imshow ("Primary_Frame",uw);

imshow ("found_gray",gray );

if (found 1 )

\{

drawChessboardCorners (gray, board_sz, corners1, found1); drawChessboardCorners (uw, board_sz, corners1, found1); printf ("found $1 \backslash \mathrm{n} ")$;

resize (uw, uw, Size ()$, 2,2$, INTER_LINEAR);

imshow ("found_gray", gray);

imshow ("found_corners",uw);

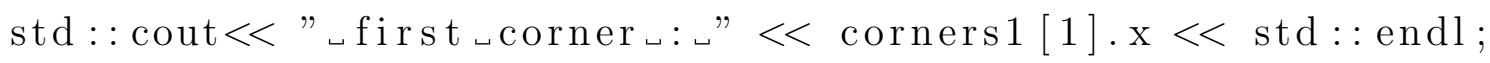
GaussianBlur (uw, uw, Size $(7,7), 1.5,1.5)$;

cvtColor (uw, edges, CVBGR2GRAY);

GaussianBlur (edges, edges, Size $(7,7), 1.5,1.5)$;

Canny (edges, edges, lowThreshold , (lowThreshold $* 3$ ), 3 ); imshow ("edges", edges ); 
///Image 2

bool found 2 false;

found 2 = findChessboardCorners (uw_2, board_sz, corners2, CV_CALIB_CB_ADAPTIVE_THRESH | CV_CALIB_CB_FILTER_QUADS );

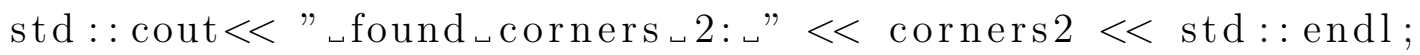

drawChessboardCorners(gray_2, board_sz, corners2, found2); drawChessboardCorners(uw_2, board_sz, corners2, found2); imshow ("Secondary_Frame", uw_2);

imshow (" found_gray_2", gray_2 );

if (found2)

\{

drawChessboardCorners(gray_2, board_sz, corners2, found2); drawChessboardCorners (uw_2, board_sz, corners2, found2); printf ("found $2 \backslash \mathrm{n} ")$;

resize (uw_2, uw_2, Size ( ), 2, 2, INTER_LINEAR);

imshow ("found_gray_2", gray_2 );

imshow ("found_corners_2", uw_2);

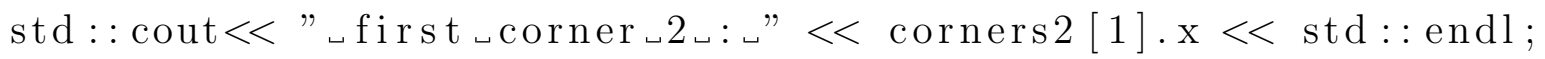

GaussianBlur (uw_2, uw_2, Size $(7,7), 1.5,1.5)$;

cvtColor (uw_2, edges_2, CVBGR2GRAY);

GaussianBlur (edges_2, edges_2, Size $(7,7), 1.5,1.5)$;

Canny (edges_2, edges_2, lowThreshold, ( lowThreshold $* 3), 3)$; 


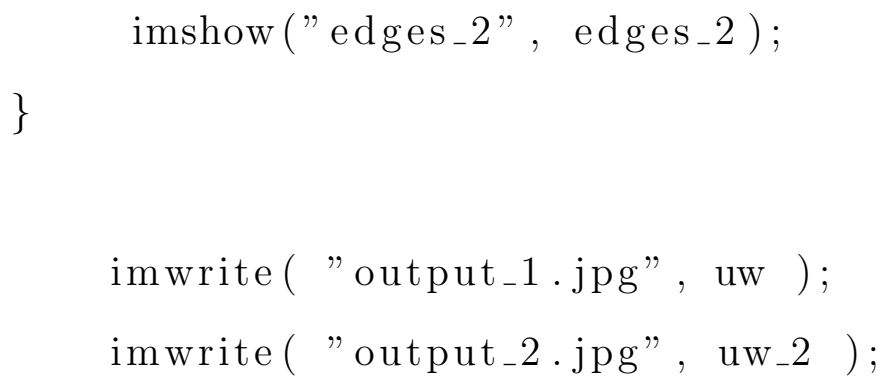




\section{List of References}

[1] S. Thrum, W. Burgrad, and D. Fox, Probabilistic Robotics. Massachusetts: The MIT Press, 2006.

[2] A. Kaebler and G. Bradski, Learning OpenCV: Computer Vision With The OpenCV Library. United states: O'Reilly Media, 2008.

[3] R. Bunschoten, Mappinng and Localization From a Panoramic Vision Sensor. PhD thesis, University of Amesterdam, Nov. 2003.

[4] R. Laganiere, OpenCV 2 Computer Vision Application Programming Cookbook. United Kingdom: Packet publishing, 2011.

[5] F. Fraundorfer and D. Scaramuzza, "Visual odometry: Part i: The first 30 years and fundamentals," IEEE Robotics and Automation Magazine, vol. 18, pp. 80-92, Dec. 2011.

[6] F. Fraundorfer and D. Scaramuzza, "Visual odometry: Part ii: Matching, robustness, optimization, and applications," IEEE Robotics and Automation Magazine, vol. 19, pp. 78-90, June 2012.

[7] R. Szeliski, Computer Vision: Algorithms and Applications. New York: Springer, 2010.

[8] R. Duda and P. Hart, "Use of the hough transformation to detect lines and curves in pictures," Comm. of the ACM, vol. 15, pp. 11-15, Sept. 1972.

[9] S. Nayar and V. Peri, "Folded catadioptric cameras," in Proc. IEEE Conf. Computer Vision and Pattern Recognition, pp. 217-223, June 1999.

[10] S. Nayar, "Catadioptric omnidirectional camera," in Proc. IEEE Conf. Computer Vision and Pattern Recognition, pp. 482-488, June 1997. 
[11] R. Swaminathan, M. D. Grossberg, and S. K. Nayar, "Non-single viewpoint catadioptric cameras: Geometry and analysis," Int. Journal of Computer Vision, vol. 66, pp. 211-229, Mar. 2006.

[12] T. Svoboda and T. Pajdla, "Central panoramic cameras: Design and geometry," in Proc. Computer Vision Winter Workshop, pp. 120-133, Feb. 1998.

[13] K. Guo, Z. Li, Image Reconstruction From Omni-Directional Camera. Technical report No. ECE-2007-06, Boston University, Dec. 2007.

[14] O. E. Kadmiri, Z. E. Kadmiri, and L. Masmoudi, "A spherical electrostatic model edge detector for omnidirectional images," Journal of Theoretical and Applied Info. Tech., vol. 51, pp. 383-391, May 2013.

[15] N. Chong, Y. Kho, and M. Wong, "Custom aspect ratio correction for unwrapped omnidirectional view images," Elsevier Computers and Electrical Engineering Journal, vol. 40, pp. 974-981, Apr. 2014.

[16] Z. Zhu, "Omnidirectionaal stereo vision," in Proc. IEEE ICAR Workshop on Omnidirectional Vision, pp. 22-25, Aug. 2001.

[17] Y. Negishi, J. Miura, and Y. Shirai, "Mobile robot navigation in unknown environments using omnidirectional stereo and laser range finder," in Proc. IEEE Int. Conf. Intelligent Robots and Systems, pp. 2737-2742, Sept. 2004.

[18] M. Tomono, "Robust 3D SLAM with a stereo camera based on an edge-point ICP algorithm," in Proc. IEEE Int. Conf. on Robotics and Automation, pp. 4306-4311, May 2009.

[19] L. Spacek and C. Burbridge, "Instantaneous robot self-localization and motion estimation with omnidirectional vision," Elsevier Robotics and Autonomous Systems Journal, vol. 55, pp. 667-674, Sept. 2007.

[20] D. Scaramuzza, A. Martinelli, and R. Siegwart, "A toolbox for easily calibrating omnidirectional cameras," in Proc. Int. Conf. Intelligent Robots and Systems, pp. 5695-5701, Oct. 2006.

[21] V. Peri and S. Nayar, "Generation of perspective and panoramic video from omnidirectional video," in Proc. DARPA Image Understanding Workshop, pp. 13841387, May 1997. 
[22] D. Lee, S. Son, K.-W. Yang, J. Park, and H. Lee, "Sensor fusion localization system for outdoor mobile robot," in ICCAS-SICE Int. Joint Conf., pp. 13841387, Aug. 2009.

[23] S. Abrahama and W. Förstner, "Fish-eye-stereo calibration and epipolar rectification," Elsevier ISPRS Journal of Photogrammetry and Remote Sensing, vol. 59, pp. 278-288, Aug. 2005.

[24] R. Bunschoten and B. Kröse, "3D scene reconstruction from cylindrical panoramic images," Elsevier Int. Symp. on Intelligent Robotic Systems, vol. 41, pp. 111-118, Nov. 2002.

[25] R. Bunschoten and B. Kröse, "Range estimation from a pair of omnidirectional images," in Proc. IEEE Int. Conf. Robotics and Automation, pp. 1174-1179, May 2001.

[26] T. Svoboda and T. Pajdla, "Epipolar geometry for central catadioptric cameras," Springer Int. Journal of Computer Vision, vol. 49, pp. 23-37, Aug. 2002.

[27] D. Scaramuzza, A. Martinelli, and R. Siegwart, "A flexible technique for accurate omnidirectional camera calibration and structure from motion," in Proc. IEEE Int. Conf. Computer Vision Systems, pp. 45-45, Jan. 2006.

[28] B. Kröse, R. Bunschoten, S. T. Hagen, B. Terwijn, and N. Vlassis, "Household robots look and learn: Environment modeling and localization from an omnidirectional vision system," IEEE Robotics Automation Magazine, vol. 11, pp. 45-52, Dec. 2004.

[29] J. Gluckman and S. K. Nayar, "Ego-motion and omnidirectional cameras," in IEEE Int. Conf. Computer Vision, pp. 999-1005, Jan 1998.

[30] B. Micusk and T. Pajdla, "Estimation of omnidirectional camera model from epipolar geometry," in Proc. IEEE Int. Conf. Computer Vision and Pattern Recognition, pp. 485-490, June 2003.

[31] ko Hariyono, Wahyono, and Kang-Hyun, "Accuracy enhancement of omnidirectional camera calibration for structure from motion," in Proc. IEEE Int. Conf. on Control, Automation and Systems, pp. 589-592, Oct. 2013.

[32] Y. Negishi, J. Miura, and Y. Shirai, "Calibration of omnidirectional stereo for mobile robots," in Proc. IEEE Int. Conf. Intelligent Robots and Systems, pp. 2600-2605, Sept. 2004. 
[33] C. Luo, L. Su, F. Zhu, and Z. Shi, "A versatile method for omnidirectional stereo camera calibration based on bp algorithm," in Advances in Neural Networks ISNN 2006 (J. Wang, Z. Yi, J. Zurada, B.-L. Lu, and H. Yin, eds.), vol. 3972 of Lecture Notes in Computer Science, pp. 383-389, Springer Berlin Heidelberg, 2006.

[34] V.-D. Hoang, D. C. Hernndez, and K.-H. Jo, "Simple and efficient method for calibration of a camera and $2 \mathrm{~d}$ laser rangefinder," in Intelligent Information and Database Systems, vol. 8397 of Lecture Notes in Computer Science, pp. 561-570, Springer International Publishing, 2014.

[35] G. L. Mariottini, S. Scheggi, F. Morbidi, and D. Prattichizzo, "An accurate and robust visual-compass algorithm for robot-mounted omnidirectional cameras," Elsevier Robotics and Autonomous Systems Journal, vol. 60, pp. 1179-1190, Sept. 2012.

[36] H.-J. Kim and J. Lee, "Stereo aoa system for indoor slam," in Proc. IEEE Int. Conf. Control, Automation and Systems, pp. 1164-1169, Oct. 2013.

[37] I. Markovic, F. Chaumette, and I. Petrovic, "Moving object detection, tracking and following using an omnidirectional camera on a mobile robot," in Proc. IEEE Int. Conf. Robotics and Automation, Feb. 2014.

[38] D. Hernndez, V.-D. Hoang, A. Filonenko, and K.-H. Jo, "Fuzzy logic guidance control systems for autonomous navigation based on omnidirectional sensing," in Modern Advances in Applied Intelligence, vol. 8481 of Lecture Notes in Computer Science, pp. 420-429, Springer International Publishing, 2014.

[39] D. Hernandez, V.-D. Hoang, A. Filonenko, and K.-H. Jo, "Vision-based heading angle estimation for an autonomous mobile robots navigation," in Proc. IEEE Int. Symp. on Industrial Electronics (ISIE), pp. 1967-1972, Jun. 2014.

[40] M. Ester, H. Kriegel, J. Sander, and X. Xu, "A density-based algorithm for discovering clusters in large spatial databases with noise," in Proc. Int. Conf. Knowledge Discovery and Data Mining, pp. 226-231, Aug. 1996.

[41] D. Hernndez, V.-D. Hoang, and K.-H. Jo, "Vanishing point based image segmentation and clustering for omnidirectional image," in Intelligent Computing Theories and Technology, vol. 7996 of Lecture Notes in Computer Science, pp. 541-550, Springer Berlin Heidelberg, 2013. 
[42] D. C. Hernndez, V.-D. Hoang, and K.-H. Jo, "Methods for vanishing point estimation by intersection of curves from omnidirectional image," in Intelligent Information and Database Systems, vol. 8397 of Lecture Notes in Computer Science, pp. 543-552, Springer International Publishing, 2014.

[43] J. Canny, "A computational approach to edge detection," IEEE Trans. on Pattern Analysis and Machine Intelligence, vol. 8, pp. 679-698, Nov. 1986.

[44] E. Mair, M. Augustine, B. Jger, A. Stelzer, C. Brand, D. Burschka, and M. Suppa, "A biologically inspired navigation concept based on the landmark-tree map for efficient long-distance robot navigation," Journal on Advanced Robotics, vol. 28, no. 5, pp. 289-302, 2014.

[45] J. Hariyono, V.-D. Hoang, and K.-H. Jo, "Human detection from mobile omnidirectional camera using ego-motion compensated," in Intelligent Information and Database Systems, vol. 8397 of Lecture Notes in Computer Science, pp. 553-560, Springer International Publishing, 2014.

[46] B. Kotze, G. Jordaan, and H. Vermaak, "Reconfigurable navigation of an automatic guided vehicle utilising omnivision," in Proc. IEEE Robotics and Mechatronics Conf., pp. 80-86, Oct. 2013.

[47] S.-Y. Chiang, X. Guo, and H.-W. Hu, "Real time self-localization of omni-vision robot by pattern match system," in Proc. IEEE Conf. Advanced Robotics and Intelligent Systems, pp. 46-50, Jun. 2014.

[48] S. Weiss, M. Achtelik, L. Kneip, D. Scaramuzza, and R. Siegwart, "Intuitive 3d maps for mav terrain exploration and obstacle avoidance," Journal of Intelligent and Robotic Systems, vol. 61, no. 1-4, pp. 473-493, 2011.

[49] C. Forster, M. Pizzoli, and D. Scaramuzza, "Svo: Fast semi-direct monocular visual odometry," in Proc. IEEE Int. Conf. on Robotics and Automation, pp. 1522, May 2014.

[50] S. Suzuki and R. Suda, "A vision system with wide field of view and collision alarms for teleoperation of mobile robots," ROBOMECH Journal, vol. 1, no. 1, 2014.

[51] S.-E. Shih and W.-H. Tsai, "Optimal design and placement of omni-cameras in binocular vision systems for accurate 3-d data measurement," IEEE Trans. on Circuits and Systems for Video Technology, vol. 23, pp. 1911-1926, Nov. 2013. 
[52] D. Herceg, I. Markovic, and I. Petrovic, "Real-time detection of moving objects by a mobile robot with an omnidirectional camera," in Proc. IEEE Int. Symp. Image and Signal Processing and Analysis, (Croatia), pp. 289-294, Sept. 2011.

[53] D. Scaramuzza, "1-point-ransac structure from motion for vehicle-mounted cameras by exploiting non-holonomic constraints," International Journal of Computer Vision, vol. 95, no. 1, pp. 74-85, 2011.

[54] A. Harmat and I. Sharf, "Towards full omnidirectional depth sensing using active vision for small unmanned aerial vehicles," in Proc. IEEE Canadian Conf. Computer and Robot Vision, pp. 24-31, May 2014.

[55] C.-H. L. Chen and M.-F. R. Lee, "Global path planning in mobile robot using omnidirectional camera," in Proc. IEEE Int. Conf. Consumer Electronics, Communications and Networks, pp. 4986-4989, Apr. 2011.

[56] O. Cogal, A. Akin, K. Seyid, V. Popovic, A. Schmid, B. Ott, P. Wellig, and Y. Leblebici, "A new omni-directional multi-camera system for high resolution surveillance," in Proc. SPIE, pp. 91200N-91200N-9, May 2014. 